\title{
Grothendieck Groups of Bundles on Varieties over Finite Fields
}

\author{
A. AGBOOLA ${ }^{1}$ and D. BURNS ${ }^{2}$ \\ ${ }^{1}$ Department of Mathematics, University of California, Santa Barbara, CA 93106, U.S.A. \\ e-mail:agboola@math.ucsb.edu \\ ${ }^{2}$ Department of Mathematics, King's College London, Strand, London WC2R 2LS, U.K. \\ e-mail: david.burns@kcl.ac.uk
}

(Received: July 2000)

Abstract. Let $X$ be an irreducible, projective variety over a finite field, and let $\mathcal{A}$ be a sheaf of rings on $X$. In this paper, we study Grothendieck groups of categories of vector bundles over certain types of ringed spaces $(X, \mathcal{A})$.

Mathematics Subject Classifications (2000): 11-XX, 19-XX, 14-XX, 13-XX.

Key words: Grothendieck groups, classgroups, sheaves of algebras, realisable classes, Witt vectors.

\section{Introduction}

Let $X$ be an irreducible, projective variety over a finite field $k$ of characteristic $p$. We write $\mathcal{O}$, (or $\mathcal{O}_{X}$ if we need to be more precise), for the structure sheaf of $X$. In this paper we shall study categories of vector bundles over certain types of ringed spaces $(X, \mathcal{A})$.

Our main interest is the case in which $X$ is a curve and $\mathcal{A}=\mathcal{O}[G]$ for a finite group $G$ (so that $\mathcal{A}(U)$ is equal to the group ring $\mathcal{O}(U)[G]$ for each open subset $U$ of $X$ ). The reason for this is that vector bundles over such ringed spaces arise naturally when one attempts to apply and extend the techniques of classical Galois module theory in a geometrical setting. For example, the study of such vector bundles underlies [Cha] and $[\mathrm{A}, \mathrm{Bu}]$, as well as the function field results of $[\mathrm{Bu}$, $\mathrm{Ch}$. It is also related to the function field case of the work of Chinburg in [Ch], and hence also to the subsequent work of Chinburg, Erez, Pappas and Taylor (see, e.g., [Ch, E ,P, T1, 2]) concerning Galois structure invariants which are associated to the de Rham cohomology of sheaves of differentials attached to tame covers of schemes (see Remark 4.5.1). Despite this, as far as we are aware, there has until now been no systematic study of the Grothendieck groups of the categories of such vector bundles.

It should be noted at the outset that the study of module categories over such ringed $(X, \mathcal{A})$ spaces is in general rather complicated. For example, the algebra of sections $\mathcal{A}(U)$ usually has a non-trivial Jacobson radical and so there are no 
analogues of the standard Wedderburn decomposition theorems. More importantly, as $X$ is not affine, several standard techniques used in the study of modules over rings (e.g. Schanuel's lemma, the Krull-Schmidt-Azumaya theorem and the devissage theorems in algebraic $K$-theory (cf. [B], Chap. VIII, § 4)) cannot be applied in the same way as in the affine case (cf. [At], for example). Despite these difficulties, our aim is to describe the Grothendieck groups of these categories in a manner which is amenable to explicit computations in interesting arithmetical contexts. Such attempts originate in [Cha] in the case that $X$ is a curve and $\mathcal{A}=\mathcal{O}[G]$ for groups $G$ with $(p, \# G)=1$. We shall, amongst other things, further clarify the results of [Cha], and also extend them to deal with arbitrary finite groups $G$.

An outline of the contents of this paper is as follows. The main purpose of Section 1 is to give basic definitions and to collect together some elementary properties of the categories with which we shall be concerned. After some preliminary remarks concerning coherent sheaves of algebras, sheaves of orders and categories of bundles, we define and discuss the class group $\mathrm{Cl}^{l f}(\mathcal{A})$ of a coherent sheaf of algebras $\mathcal{A}$. (The superscript ' $l f^{\prime}$ ' refers to the fact that the class group is defined in terms of modules which are locally free, rather than locally projective.) In the case that $X$ is a curve, we describe a version of Quillen's localisation theorem which is valid in our context, and for coherent sheaves of commutative algebras $\mathcal{A}$, we describe the connection between $\operatorname{Cl}^{l f}(\mathcal{A})$ and the $\operatorname{Picard}$ group $\operatorname{Pic}(\mathcal{A})$ of $\mathcal{A}$.

The first part of Section 2 is devoted to a fairly detailed analysis of the structure of the Grothendieck group $K_{0}^{l f}(\mathcal{A})$ of the category of $\mathcal{A}$-bundles on $X$. We begin by defining a series of 'kernel subgroups' of $K_{0}^{l f}(\mathcal{A})$. These subgroups give rise to a natural decreasing filtration on $K_{0}^{l f}(\mathcal{A})$, and we investigate some properties of this filtration. Next, we apply the results of Section 1 to give an idelic description of $\mathrm{Cl}^{l f}(\mathcal{A})$ in the case that $X$ is a curve and $\mathcal{A}$ is a sheaf of orders on $X$. We then deduce a number of consequences of this description. For example, we apply the idelic description to investigate $\mathrm{Cl}^{l f}(\mathcal{O}[G])$ in the case that $p \nmid \# G$. Our results in this case clarify and extend those of Chapman in [Cha]. We conclude Section 2 by addressing the case in which $\mathcal{A}=\mathcal{O}[G]$ for any finite Abelian group $G$. We describe $\operatorname{Pic}(\mathcal{O}[G])$ in terms of the Picard scheme of $X$, and we show that the 'kernel subgroups' referred to above can be reinterpreted in terms of natural coinflation maps on $K_{0}^{l f}(\mathcal{O}[G])$.

We now restrict ourselves to the case in which $\mathcal{A}=\mathcal{O}[G]$, where $G$ is a cyclic group of order $p^{n}$. In Section 3, we describe $\operatorname{Pic}(\mathcal{O}[G])$ in terms of Serre's Witt vector cohomology (cf. [S1]), and we prove various functorial properties of this description. These functorial properties occur repeatedly in applications to, for example, Galois module theory. We then apply our techniques to describe those elements of $\mathrm{Cl}^{l f}(\mathcal{O}[G])$ which arise from structure sheaves of unramified $G$ covers $Y \rightarrow X$. The results of this section clarify and go beyond those of $[\mathrm{A}, \mathrm{Bu}]$. 


\section{Generalities}

Let $k$ be a finite field of characteristic $p$, and $k^{c}$ a fixed algebraic closure of $k$.

Throughout this paper, unless explicitly stated otherwise, $X$ denotes an irreducible, projective variety over $k$. A curve is an irreducible, non-singular projective variety of dimension one over $k$. We write $\mathcal{O}$ (or $\mathcal{O}_{X}$ if we need to be more precise) for the structure sheaf of $X$, and we assume that $k$ is equal to the field of constants $\mathcal{O}(X)$ of $X$. We write $K$ (or $k(X)$ if we need to be more precise) for the function field of $X$. Recall that, under the above assumptions, $X$ has a unique generic point $\eta$ and $K$ is by definition equal to the stalk of $\mathcal{O}$ at $\eta$. In addition, if $U$ is any affine open subset of $X$, then $\mathcal{O}(U)$ is an integral domain (a Dedekind domain if $X$ is a curve) and $K$ is canonically isomorphic to its quotient field. We write $X_{0}$ for the set of closed points of $X$.

For any ring $R$ we let $\operatorname{Jac}(R)$ denote its Jacobson radical. We recall that $\operatorname{Jac}(R)$ is defined to be the intersection over all maximal left ideals of $R$, and so is in fact a two-sided ideal of $R$. All modules are left modules, unless explicitly stated otherwise. We write $\mathfrak{B}^{p}(R)$ and $\mathfrak{B}^{f}(R)$ for the categories of finitely generated projective and finitely generated free $R$-modules respectively. We denote the group of units of $R$ by $R^{*}$.

In this section we shall give the basic definitions and collect together some basic properties of the categories with which we are concerned.

\section{SHEAVES OF ORDERS}

Let $\mathcal{A}$ be a sheaf of rings on $X$, and write $A$ for its generic stalk. We say that $\mathcal{A}$ is a coherent sheaf of algebras, respectively a sheaf of orders, if it is a sheaf of $\mathcal{O}$-algebras which is coherent, respectively locally-free, when regarded as a sheaf of $\mathcal{O}$-modules. We observe that if $\mathcal{A}$ is a sheaf of orders, then there is an affine open covering $\mathcal{U}$ of $X$ such that for each $U$ in $\mathcal{U}$, the algebra of sections $\mathcal{A}(U)$ is an $\mathcal{O}(U)$-order in the finite dimensional $K$-algebra $A$ in the sense of ([C,R1], § 23).

If $\mathcal{V}$ is any $\mathcal{O}$-module we write $\mathcal{V}_{x}$ for the stalk of $\mathcal{V}$ at a point $x$ of $X$.

DEFINITION 1.1. Let $\mathcal{A}$ be a coherent sheaf of algebras on $X$. We define the radical $\mathcal{R}(\mathcal{A})$ of $\mathcal{A}$ to be the unique maximal sheaf of two-sided ideals of $\mathcal{A}$ which is coherent as an $\mathcal{O}$-module and is such that $\mathcal{R}(\mathcal{A})_{x} \subseteq \operatorname{Jac}\left(\mathcal{A}_{x}\right)$ for all points $x \in X$. We shall frequently write $\mathcal{R}$ instead of $\mathcal{R}(\mathcal{A})$.

Remark 1.2. If $\mathcal{A}$ is a coherent sheaf of algebras on any Noetherian scheme $X$, then the existence of a sheaf $\mathcal{R}(\mathcal{A})$ as above may be shown as follows. The set $\left\{\mathcal{R}_{j}\right\}$ of two-sided ideals of $\mathcal{A}$ which are coherent as $\mathcal{O}$-modules and are such that $\mathcal{R}_{j, x} \subseteq \operatorname{Jac}\left(\mathcal{A}_{x}\right)$ for all $x \in X$ is non-empty, since it contains the zero sheaf of ideals. For each open subset $U$ of $X$, let $\sum_{j} \mathcal{R}_{j}(U)$ denote the two-sided ideal of $\mathcal{A}(U)$ generated by the set $\left\{\mathcal{R}_{j}(U)\right\}$. Let $\mathcal{R}(\mathcal{A})$ be the subsheaf of $\mathcal{A}$ which is 
obtained by sheafifying the presheaf which has sections $\sum_{j} \mathcal{R}_{j}(U) \subset \mathcal{A}(U)$ for each open subset $U$ of $X$. It remains to show that $\mathcal{R}(\mathcal{A})$ is a coherent $\mathcal{O}$-module. Since $X$ is Noetherian, this follows because $\mathcal{R}(\mathcal{A})$ is a submodule of the coherent $\mathcal{O}$-module $\mathcal{A}$. This last fact seems to be well known, but we were unable to find a satisfactory reference and so we shall quickly sketch a proof. Let $U=\operatorname{Spec}(\Lambda)$ be an open affine subset of $X$. For each index $j,\left.\mathcal{R}_{j}\right|_{U}$ is the sheaf $\tilde{M}_{j}$ associated to a finitely generated $\Lambda$-submodule $M_{j}$ of $\mathcal{A}(U)$. It follows that $\left.\mathcal{R}(\mathcal{A})\right|_{U}$ is the sheafification of the presheaf $\sum_{j} \tilde{M}_{j}$, and hence is the sheaf associated to the ideal $\sum_{j} M_{j}$ of $\mathcal{A}(U)$ (cf. for example [D,Gr], I, (1.3.9)). This ideal is a finitely generated $\Lambda$-module since $\mathcal{A}(U)$ is finitely generated and $\Lambda$ is Noetherian (since $X$ is).

DEFINITION 1.3. Let $\mathcal{A}$ be a coherent sheaf of algebras on $X$, and let $\mathcal{J}(\mathcal{A})$ denote the maximal sheaf of two-sided ideals of $\mathcal{A}$ which is coherent as an $\mathcal{O}$-module and such that $\mathcal{J}(\mathcal{A})_{x} \subseteq \operatorname{Jac}\left(\mathcal{A}_{x}\right)$ for all closed points $x \in X_{0}$. (The existence and uniqueness of $\mathcal{J}(\mathcal{A})$ may be shown by an argument similar to that given for the sheaf $\mathcal{R}(\mathcal{A})$ in the Remark 1.2).

Note that $\mathcal{J}(\mathcal{A})$ is defined in terms of just the closed points of $X$, while $\mathcal{R}(\mathcal{A})$ is defined in terms of all of the points of $X$. The following result shows that if $X$ is a curve or $\mathcal{A}$ is a sheaf of orders, then $\mathcal{R}(\mathcal{A})$ is in fact equal to $\mathcal{J}(\mathcal{A})$.

For each non-negative integer $i$ and each sheaf of two-sided ideals $\mathcal{I}$ of $\mathcal{A}$ we write $\mathcal{I}^{i}$ for the subsheaf of $\mathcal{A}$ which has stalks $\left(\mathcal{I}_{x}\right)^{i}$ for each $x \in X$. (Here we use the convention $\left(\mathcal{I}_{x}\right)^{0}=\mathcal{A}_{x}$ for each $x$.)

LEMMA 1.4. Let $\mathcal{A}$ be a coherent sheaf of algebras on $X$, and set $N:=\operatorname{dim}_{K}\left(\mathcal{A}_{\eta}\right)$.

(a) $\mathcal{J}(\mathcal{A})^{N}$ is a torsion sheaf. Hence $\mathcal{J}(\mathcal{A})_{\eta} \subseteq \operatorname{Jac}\left(\mathcal{A}_{\eta}\right)$.

(b) If $X$ is a curve, then $\mathcal{J}(\mathcal{A})=\mathcal{R}(\mathcal{A})$.

(c) If $\mathcal{A}$ is a sheaf of orders, then $\mathcal{J}(\mathcal{A})=\mathcal{R}(\mathcal{A})$ and $\mathcal{R}(\mathcal{A})^{N}$ is the zero sheaf.

Proof. We write $\mathcal{J}$ and $\mathcal{R}$ in place of $\mathcal{J}(\mathcal{A})$ and $\mathcal{R}(\mathcal{A})$, respectively.

If $\left(\mathcal{J}^{N}\right)_{\eta}=\{0\}$, then $\mathcal{J}_{\eta}$ is nilpotent and so certainly belongs to $\operatorname{Jac}\left(\mathcal{A}_{\eta}\right)$. To prove (a) it therefore suffices to prove that $\mathcal{J}^{N}$ is a torsion sheaf. Let $\mathcal{T}$ denote the two-sided ideal of $\mathcal{A}$ for which $\mathcal{T}_{x}$ is equal to the $\mathcal{O}_{x}$-torsion submodule of $\mathcal{A}_{x}$ for each $x \in X$. Set $\mathcal{A}_{1}:=\mathcal{A} / \mathcal{T}$ and let $\mathcal{J}_{1}$ denote the image of $\mathcal{J}$ in $\mathcal{A}_{1}$. Then $\mathcal{A}_{1}$ is a coherent sheaf of algebras on $X$ and $\mathcal{J}_{1}$ is a two-sided ideal of $\mathcal{A}_{1}$, coherent as an $\mathcal{O}$-module, and such that $\mathcal{J}_{1, x} \subseteq \operatorname{Jac}\left(\mathcal{A}_{1, x}\right)$ for each $x \in X_{0}$. By replacing $\mathcal{A}$ and $\mathcal{J}$ by $\mathcal{A}_{1}$ and $\mathcal{J}_{1}$ we may therefore assume that $\mathcal{A}(U)$ is $\mathcal{O}(U)$-torsion-free for each affine open subset $U$ of $X$. Under this condition, we need to prove that $\mathcal{J}^{N}(U)=\{0\}$ for each such $U$.

Let $U=\operatorname{Spec}(R)$, and set $S:=\mathcal{A}(U)$ and $J:=\mathcal{J}(U)$. Closed points of $U$ correspond bijectively to maximal ideals of $R$. If $\mathfrak{m}$ is any such ideal, then the $k$ algebra $S / \mathfrak{m}$ is finitely generated and $\operatorname{so} \operatorname{Jac}(S / \mathfrak{m})$ is nilpotent. Let $m>0$ be an 
integer such that $\operatorname{Jac}(S / \mathfrak{m})^{m}=\{0\}$. Since $J \subseteq \mathcal{J}_{\mathfrak{m}} \subseteq \operatorname{Jac}\left(S_{\mathfrak{m}}\right)$ and this projects into $\operatorname{Jac}\left(S_{\mathfrak{m}} / \mathfrak{m} S_{\mathfrak{m}}\right)=\operatorname{Jac}(S / \mathfrak{m})$, it follows that $J^{m} \subseteq \mathfrak{m} S$. This inclusion is valid for all maximal ideals $\mathfrak{m}$ of $R$, and we claim this implies that $J^{m}=\{0\}$. To show this, we first observe that $R$ is a Jacobson ring, that is, every prime ideal of $R$ is the intersection of a family of maximal ideals of $R$ (see [Bo], Chap. V, $\S 3.4$, Def. 1 and Cor. 1). This implies that the intersection over all maximal ideals of $R$ is equal to its nilradical (cf. [Bo], Chap. V, §3.4, Prop. 3), and hence is equal to $\{0\}$ since $R$ is a domain. It follows that $f\left(J^{m}\right)=\{0\}$ for any homomorphism $f \in \operatorname{Hom}_{R}(S, R)$. However, if $J^{m}$ is non-zero, then by extending any non-zero element of $J^{m}$ to a $K$-basis of the space $S \otimes_{R} K$ it is easy to produce a homomorphism $f \in \operatorname{Hom}_{R}(S, R)$ with $f\left(J^{m}\right) \neq\{0\}$. It follows that $J^{m}=\{0\}$ and hence that $J \subseteq \operatorname{Jac}\left(\mathcal{A}_{\eta}\right)$. Since $\operatorname{Jac}\left(\mathcal{A}_{\eta}\right)^{N}=\{0\}$ with $N$ equal to the $K$-dimension of $\mathcal{A}_{\eta}$, it follows that $J^{N}=\{0\}$. This completes the proof of (a).

If $X$ is a curve, then $X=X_{0} \cup\{\eta\}$. Hence (a) implies that $\mathcal{J}(\mathcal{A})_{x} \subseteq \operatorname{Jac}\left(\mathcal{A}_{x}\right)$ for all $x \in X$, and so it is obvious that $\mathcal{J}(\mathcal{A})=\mathcal{R}(\mathcal{A})$. This proves (b).

If $\mathcal{A}$ is a sheaf of orders, then there is an affine open covering $\mathcal{U}=\left\{U_{i}\right\}$ of $X$ such that each $\mathcal{A}\left(U_{i}\right)$ is a free $\mathcal{O}\left(U_{i}\right)$-module, and hence embeds into $\mathcal{A}_{\eta}$. Since $\mathcal{J}^{N}\left(U_{i}\right)$ is a submodule of $\mathcal{A}\left(U_{i}\right)$ which has zero image in $\mathcal{A}_{\eta}$ (by (a)) we must have $\mathcal{J}^{N}\left(U_{i}\right)=\{0\}$. For each $x \in X$ it follows that $\left(\mathcal{J}_{x}\right)^{N}=\left(\mathcal{J}^{N}\right)_{x}=\{0\}$ and hence that $\mathcal{J}_{x} \subseteq \operatorname{Jac}\left(\mathcal{A}_{x}\right)$. Therefore $\mathcal{J}(\mathcal{A})=\mathcal{R}(\mathcal{A})$ and so $\mathcal{R}(\mathcal{A})^{N}$ is the zero sheaf. This proves (c).

DEFINITION 1.5. If $\mathcal{A}$ is any coherent sheaf of algebras on $X$, then for each nonnegative integer $i$ we write $\mathcal{A}_{s s}^{(i)}$ for the coherent sheaf of algebras which has stalks $\mathcal{A}_{x} /\left(\mathcal{R}_{x}\right)^{i}$ for each $x \in X$. We write $\mathcal{A}_{s s}$ in place of $\mathcal{A}_{s s}^{(1)}$.

EXAMPLES 1.6. (a) Let $G$ be a finite group and let $\mathcal{A}$ denote the sheaf of orders $\mathcal{O}[G]$. Then $\mathcal{A}=\mathcal{O} \otimes_{k} k[G]$, and for each integer $i \geqslant 0$ one has $\mathcal{R}(\mathcal{A})^{i}=$ $\mathcal{O} \otimes_{k} \operatorname{Jac}(k[G])^{i}$ and $\mathcal{A}_{s s}^{(i)}=\mathcal{O} \otimes_{k}\left(k[G] / \operatorname{Jac}(k[G])^{i}\right.$ ) (see the proof of Lemma 1.8 below). In particular, $\mathcal{A}_{s s}(X)$ is a semisimple $k$-algebra.

(b) The following example was shown to us by D. Grayson. Let $X=\mathbb{P}_{k}^{1}$. Let $\mathcal{A}$ be the subsheaf of the constant sheaf of $2 \times 2$-matrices over $k(X)$ which is defined by

$$
\mathcal{A}=\left(\begin{array}{cc}
\mathcal{O} & \mathcal{O}(1) \\
\mathcal{O}(-1) & \mathcal{O}
\end{array}\right)
$$

Then $\mathcal{A}$ is a sheaf of orders. Also, $\operatorname{Jac}\left(\mathcal{A}_{x}\right)=0$ for each $x \in X$ so that $\mathcal{R}(\mathcal{A})=\{0\}$ and hence $\mathcal{A}_{s s}^{(i)}=\mathcal{A}$ for each integer $i \geqslant 0$. In particular, the $k$-algebra $\mathcal{A}_{s s}(X)=$ $\mathcal{A}(X)$ is not semisimple.

The above examples prompt us to make the following definition.

DEFINITION 1.7. We shall say that a sheaf of $\mathcal{A}$-modules $\mathcal{V}$ is isotrivial if

$$
\mathcal{V}(U)=\mathcal{O}(U) \otimes_{k} \mathcal{V}(X)
$$


for each open subset $U$ of $X$. Observe that the sheaf $\mathcal{A}=\mathcal{O}[G]$ of Example 1.6(a) is isotrivial, while the sheaf considered in Example 1.6(b) is not.

If a coherent sheaf of algebras is isotrivial, then it is a sheaf of orders. Our next result describes certain key properties of isotrivial sheaves of algebras (and in particular proves all claims in Example 1.6(a)).

For any positive integer $n$, we let $k_{n}$ denote the unique extension of $k$ of degree $n$ in $k^{c}$, we set $X_{n}:=X \times_{k} k_{n}$, and we let $f_{n}: X_{n} \rightarrow X$ denote the natural morphism. Since there are no non-commutative finite division rings, there is a finite index set $I$, and strictly positive integers $n(i)$ and $m(i)$ for each $i \in I$ such that the Wedderburn decomposition of the semisimple $k$-algebra $\mathcal{A}(X) / \operatorname{Jac}(\mathcal{A}(X))$ has the form

$$
\mathcal{A}(X) / \operatorname{Jac}(\mathcal{A}(X)) \cong \prod_{i \in I} M_{n(i)}\left(k_{m(i)}\right)
$$

LEMMA 1.8. Let $\mathcal{A}$ be any isotrivial sheaf of algebras on $X$, and set $J:=$ $\operatorname{Jac}(\mathcal{A}(X))$.

(a) If $i$ is any non-negative integer, then $\mathcal{R}(\mathcal{A})^{i}=\mathcal{O} \otimes_{k} J^{i}$ and $\mathcal{A}_{s s}^{(i)} \cong \mathcal{O} \otimes_{k}$ $\left(\mathcal{A}(X) / J^{i}\right)$.

(b) The k-algebra $\mathcal{A}_{s s}(X)$ is isomorphic to $\mathcal{A}(X) / J$ and is therefore semisimple. If $(1.1)$ is the Wedderburn decomposition of $\mathcal{A}_{s s}(X)$, then

$$
\mathcal{A}_{s s} \cong \prod_{i \in I} M_{n(i)}\left(\left(f_{m(i)}\right)_{*} \mathcal{O}_{X_{m(i)}}\right),
$$

where here $M_{n(i)}\left(\left(f_{m(i)}\right)_{*} \mathcal{O}_{X_{m(i)}}\right)$ denotes the sheaf on $X$ whose sections over any open subset $U$ are the elements of the matrix ring $M_{n(i)}\left(\mathcal{O}_{X_{m(i)}}\left(f_{m(i)}^{-1} U\right)\right)$.

Proof. We set $\mathcal{R}_{1}:=\mathcal{O} \otimes_{k} J, S:=\mathcal{A}(X) / J$ and $\mathcal{R}:=\mathcal{R}(\mathcal{A})$. All claims in (a) follow easily if we can show that $\mathcal{R}=\mathcal{R}_{1}$. But the inclusion $\mathcal{R}_{1} \subseteq \mathcal{R}$ is clear since $J$ is nilpotent and, on the other hand, Lemma 1.4(c) implies that $\mathcal{R}(U) \subseteq \operatorname{Jac}(\mathcal{A}(U))$ for each open subset $U$ of $X$. It is therefore sufficient to prove that $\operatorname{Jac}(\mathcal{A}(U)) \subseteq \mathcal{R}_{1}(U)$, or equivalently that $\operatorname{Jac}\left(\mathcal{A}(U) / \mathcal{R}_{1}(U)\right)=\{0\}$, for each affine open subset $U$ of $X$. Note that the decomposition (1.1) induces an isomorphism

$$
\begin{aligned}
\mathcal{A}(U) / \mathcal{R}_{1}(U) & \cong \mathcal{O}(U) \otimes_{k} S \\
& \cong \prod_{i \in I} M_{n(i)}\left(\mathcal{O}(U) \otimes_{k} k_{m(i)}\right)
\end{aligned}
$$

so that

$$
\operatorname{Jac}\left(\mathcal{A}(U) / \mathcal{R}_{1}(U)\right) \cong \prod_{i \in I} M_{n(i)}\left(\operatorname{Jac}\left(\mathcal{O}(U) \otimes_{k} k_{m(i)}\right)\right)
$$

(cf. [C, R1], Prop. 5.14). It is thus sufficient to prove that $\operatorname{Jac}\left(\mathcal{O}(U) \otimes_{k} k_{m(i)}\right)=$ $\{0\}$, and this follows by an argument similar to that used in the proof of Lemma 1.4(a). 
We have now proved (a). Since (by our standing assumption) $k=\mathcal{O}(X)$, the first claim in (b) follows immediately from (a). Finally we observe that (1.2) follows from the isomorphisms

$$
\begin{aligned}
\mathcal{A}_{s s} & \cong \mathcal{O} \otimes_{k} S \\
& \cong \prod_{i \in I} \mathcal{O} \otimes_{k} M_{n(i)}\left(k_{m(i)}\right) \\
& \cong \prod_{i \in I} M_{n(i)}\left(\left(f_{m(i)}\right)_{*} \mathcal{O}_{X_{m(i)}}\right)
\end{aligned}
$$

\section{CATEgORIES OF $\mathcal{A}$-BUNDLES}

Let $\mathcal{A}$ be a coherent sheaf of algebras on $X$. We let $\operatorname{Coh}(\mathcal{A})$ denote the full subcategory of the category of $\mathcal{A}$-modules which has as objects those $\mathcal{A}$-modules for which the underlying $\mathcal{O}$-module is coherent. If $\mathcal{V}$ belongs to $\mathfrak{C} o h(\mathcal{A})$ then, since $X$ is Noetherian, the restriction of $\mathcal{V}$ to any affine open subset $U$ of $X$ is the sheaf associated to a finitely generated $\mathcal{A}(U)$-module.

DEFINITION 2.1. (a) An object $\mathcal{V}$ of $\mathfrak{C o h}(\mathcal{A})$ is a (left) projective $\mathcal{A}$-bundle if $\mathcal{V}_{x}$ is a projective $\mathcal{A}_{x}$-module for each $x \in X$. We let $\mathfrak{B}^{l p}(\mathcal{A})$ denote the full subcategory of $\operatorname{Coh}(\mathcal{A})$ consisting of projective $\mathcal{A}$-bundles.

(b) An object $\mathcal{V}$ of $\mathfrak{B}^{l p}(\mathcal{A})$ is a (left) $\mathcal{A}$-bundle if it is a locally free $\mathcal{A}$-module of constant rank. This means that $\mathcal{V}_{x}$ is a free $\mathcal{A}_{x}$-module of fixed rank for each $x \in X$. We let $\mathfrak{B}^{l f}(\mathcal{A})$ denote the full subcategory of $\mathfrak{B}^{l p}(\mathcal{A})$ consisting of $\mathcal{A}$ bundles. The rank of an $\mathcal{A}$-bundle $\mathcal{V}$ is the $\mathcal{A}_{x}$-rank of $\mathcal{V}_{x}$ for any (and therefore every) $x \in X$. For each natural number $n$ we let $\mathfrak{B}_{n}(\mathcal{A})$ denote the full subcategory of $\mathfrak{B}^{l f}(\mathcal{A})$ which has as objects those $\mathcal{A}$-bundles which are of rank $n$.

Our current investigations have been principally motivated by the results and techniques of classical Galois module theory, and accordingly the main focus of this paper concerns categories of the form $\mathfrak{B}^{l f}(\mathcal{A})$ rather than $\mathfrak{B}^{l p}(\mathcal{A})$.

DEFINITION 2.2. For each $\mathcal{V} \in \mathfrak{C} o h(\mathcal{A})$ and each integer $i \geqslant 0$ we let $\mathcal{V}_{s s}^{(i)}$ denote the $\mathcal{A}_{s s}^{(i)}$-module $\mathcal{A}_{s s}^{(i)} \otimes_{\mathcal{A}} \mathcal{V}$, and we write $\mathcal{V}_{s s}$ in place of $\mathcal{V}_{s s}^{(1)}$.

LEMMA 2.3. (a) Let $n$ be a natural number. If $\mathcal{V} \in \mathfrak{B}_{n}(\mathcal{A})$, then $\mathcal{V}_{s s}^{(i)} \in \mathfrak{B}_{n}\left(\mathcal{A}_{s s}^{(i)}\right)$ for any integer $i \geqslant 1$.

(b) Suppose that $X$ is a curve. For each point $x \in X_{0}$, let $\pi_{x}$ be a uniformiser of the discrete valuation ring $\mathcal{O}_{x}$, and let $i$ be any positive integer which is such that $\operatorname{Jac}\left(\mathcal{A}_{x}\right)^{i} \subseteq \pi_{x} \mathcal{A}_{x}$ for all $x \in X_{0}$. If $\mathcal{V}_{s s}^{(i)} \in \mathfrak{B}^{l f}\left(\mathcal{A}_{s s}^{(i)}\right)$, then $\mathcal{V} \in \mathfrak{B}^{l f}(\mathcal{A})$.

Proof. The key point here is that $\mathcal{A}_{s s, x}^{(i)}=\mathcal{A}_{x} /\left(\mathcal{R}_{x}\right)^{i}$ for each $x \in X$.

Claim (a) follows from the fact that if $\mathcal{V}_{x}$ is a free $\mathcal{A}_{x}$-module, then $\mathcal{V}_{s s, x}^{(i)}$ is a free $\mathcal{A}_{s s, x}^{(i)}$-module of the same rank. 
To prove (b) we recall that $\left(\mathcal{R}_{x}\right)^{i} \subseteq \operatorname{Jac}\left(\mathcal{A}_{x}\right)^{i}$ for each $x \in X_{0}$. Hence, it may be shown by a standard argument involving Nakayama's lemma that if $\mathcal{V}_{s s, x}^{(i)}$ is free as an $\mathcal{A}_{s s, x}^{(i)}$-module, then $\mathcal{V}_{x}$ is a free $\mathcal{A}_{x}$-module of the same rank (cf. [C,R1] (30.11)).

We shall have need of the following result, which is an immediate consequence of ([Gi], Chap. III, Cor. 2.5.2(I)).

LEMMA 2.4. For each natural number $n$ there is a canonical bijection between the set of isomorphism classes of objects in $\mathfrak{B}_{n}(\mathcal{A})$ and the pointed set $H^{1}\left(X_{Z a r}, G L_{n}(\mathcal{A})\right)$ under which the isomorphism class of $\mathcal{A}^{n}$ corresponds to the distinguished element of $H^{1}\left(X_{Z a r}, G L_{n}(\mathcal{A})\right)$.

We shall also use the following 'symmetrisation lemma'.

LEMMA 2.5. For each object $\mathcal{F}$ of $\mathfrak{C} o h(\mathcal{A})$ there exists an $\mathcal{A}$-bundle $\mathcal{V}$ and a surjective morphism $\mathcal{V} \longrightarrow \mathcal{F}$ in $\mathfrak{C} o h(\mathcal{A})$.

Proof. Since $X$ is projective there is a finite direct sum of twisted structure sheaves $\mathcal{P}$ and a surjection $\mathcal{P} \rightarrow \mathcal{F}$ of $\mathcal{O}$-modules ([H], Chap. II, (5.18)). By tensoring (over $\mathcal{O}$ ) with $\mathcal{A}$ we obtain a surjective map $\tau: \mathcal{A} \otimes \mathcal{P} \rightarrow \mathcal{A} \otimes \mathcal{F}$ in $\operatorname{Coh}(\mathcal{A})$, where $\mathcal{A} \otimes \mathcal{P} \in \mathfrak{B}^{l f}(\mathcal{A})$.

Next, we observe that, for each $x \in X$, we have a surjection $\delta_{x}: \mathcal{A}_{x} \otimes \mathcal{F}_{x} \rightarrow$ $\mathcal{F}_{x}$ given by $\sum_{j} \alpha_{x, j} \otimes f_{x, j} \mapsto \sum_{j} \alpha_{x, j}\left(f_{x, j}\right),\left(\alpha_{x, j} \in \mathcal{A}_{x}, f_{x, j} \in \mathcal{F}_{x}\right)$. We let $\delta: \mathcal{A} \otimes \mathcal{F} \rightarrow \mathcal{F}$ denote the unique surjection in $\operatorname{Coh}(\mathcal{A})$ which induces the maps $\delta_{x}$ on stalks. The surjective map which we seek is then given by the composite $\delta \circ \tau: \mathcal{A} \otimes \mathcal{P} \rightarrow \mathcal{F}$.

Finally, we record the following important fact.

LEMMA 2.6. The subcategory $\mathfrak{B}^{l f}(\mathcal{A})$ is cofinal in $\mathfrak{B}^{l p}(\mathcal{A})$.

Proof. We must show that for any object $\mathcal{V}_{1}$ of $\mathfrak{B}^{l p}(\mathcal{A})$ there exists an object $\mathcal{V}_{2}$ of $\mathfrak{B}^{l p}(\mathcal{A})$ such that the direct sum of $\mathcal{V}_{1}$ and $\mathcal{V}_{2}$ belongs to $\mathfrak{B}^{l f}(\mathcal{A})$.

It follows from Lemma 2.5 that there exists an $\mathcal{A}$-bundle $\mathcal{P}$ and a surjective map of $\mathcal{A}$-modules $\mathcal{P} \rightarrow \mathcal{V}_{1}$. Letting $\mathcal{V}_{2}$ denote the kernel of this morphism, one has a short exact sequence in $\operatorname{Coh}(\mathcal{A})$

$$
0 \longrightarrow \mathcal{V}_{2} \longrightarrow \mathcal{P} \longrightarrow \mathcal{V}_{1} \longrightarrow 0
$$

Let $x \in X$. Since $\mathcal{V}_{1, x}$ is a projective $\mathcal{A}_{x}$-module, the associated short exact sequence of stalks is split and so there is an isomorphism of $\mathcal{A}_{x}$-modules between $\mathcal{V}_{2, x} \oplus \mathcal{V}_{1, x}$ and $\mathcal{P}_{x}$. Since $\mathcal{P}_{x}$ is a free $\mathcal{A}_{x}$-module of rank independent of $x$, it follows that $\mathcal{V}_{2}$ is a projective $\mathcal{A}$-bundle and that $\mathcal{V}_{2} \oplus \mathcal{V}_{1}$ is an $\mathcal{A}$-bundle. 


\section{ALGEBRAIC $K$-GROUPS}

Suppose that $\mathcal{A}$ is a coherent sheaf of algebras on $X$. In this section we discuss some relevant properties of the algebraic $K$-groups which are attached to categories of the form $\mathfrak{B}^{l f}(\mathcal{A})$ and $\mathfrak{B}^{l p}(\mathcal{A})$.

We remark that the categories $\mathfrak{B}^{l f}(\mathcal{A})$ and $\mathfrak{B}^{l p}(\mathcal{A})$, and also $\mathfrak{B}^{f}(R)$ and $\mathfrak{B}^{p}(R)$ for any ring $R$ are all exact categories in the sense of [Q] (when endowed with the usual notion of exact sequence in each case). For each integer $i \geqslant 0$, we may therefore let $K_{i}^{l f}(\mathcal{A}), K_{i}^{l p}(\mathcal{A}), K_{i}^{f}(R)$ and $K_{i}(R)$ denote the Quillen $K$-groups in dimension $i$ of the categories $\mathfrak{B}^{l f}(\mathcal{A}), \mathfrak{B}^{l p}(\mathcal{A}), \mathfrak{B}^{f}(R)$ and $\mathfrak{B}^{p}(R)$, respectively. When $i=0$, each of the above $K$-groups coincides with the Grothendieck group of the respective category (cf. [Q], $\S 2$, Th. 1). We recall also that, by Gersten's cofinality theorem (cf. [G]), the natural 'forgetful' morphism $K_{i}^{f}(R) \rightarrow K_{i}(R)$ is bijective if $i>0$ and injective if $i=0$.

We write $A$ for the generic stalk of $\mathcal{A}$.

DEFINITION 3.1. (a) The class group $\operatorname{Cl}^{l f}(\mathcal{A})$ of the category $\mathfrak{B}^{l f}(\mathcal{A})$ is defined to be the kernel of the composite map $K_{0}^{l f}(\mathcal{A}) \rightarrow K_{0}^{f}(A) \cong \mathbb{Z}$, where the first map is induced by taking generic stalks, and the second map is induced by taking ranks over $A$. Note that this composite map is canonically split (by the map sending 1 to the class of $\mathcal{A}$ ) and so one can also regard $\mathrm{Cl}^{l f}(\mathcal{A})$ as being the quotient of $K_{0}^{l f}(\mathcal{A})$ by the subgroup generated by $(\mathcal{A})$.

(b) The class group $\mathrm{Cl}^{l p}(\mathcal{A})$ of the category $\mathfrak{B}^{l p}(\mathcal{A})$ is defined to be the kernel of the map $K_{0}^{l p}(\mathcal{A}) \rightarrow K_{0}(A)$ which is induced by taking generic stalks.

Remark 3.2. If $\mathcal{A}$ is a sheaf of orders, then there is an important distinction between the group $\mathrm{Cl}^{l f}(\mathcal{A})$ and classical locally free class groups. To describe this, for each affine open subset $U$ of $X$, we let $\mathfrak{B}^{l f}(\mathcal{A}(U))$ denote the full subcategory of $\mathfrak{B}^{p}(\mathcal{A}(U))$ which has as objects those modules $M$ such that $M \otimes_{\mathcal{O}(U)} \mathcal{O}_{x}$ is a free $\mathcal{A}_{x}$-module for all $x \in U$. The reduced class group $S K_{0}\left(\mathfrak{B}^{l f}(\mathcal{A}(U))\right)$ of the category $\mathfrak{B}^{l f}(\mathcal{A}(U))$ is defined to be the kernel of the map $K_{0}\left(\mathcal{B}^{l f}(\mathcal{A}(U))\right) \rightarrow$ $K_{0}(A)$ given by extension of scalars. Let $\mathfrak{B}_{1}^{l f}(\mathcal{A}(U))$ denote the category of locally free, rank one $\mathcal{A}(U)$-modules.

If $\mathcal{O}(U)$ is a Dedekind domain and $\mathcal{A}(U)$ is an $\mathcal{O}(U)$-order in a separable $K$ algebra, then each element of $S K_{0}\left(\mathfrak{B}^{l f}(\mathcal{A}(U))\right)$ is of the form $(\mathcal{V}(U))-(\mathcal{A}(U))$ for some $\mathcal{V}(U) \in \mathfrak{B}_{1}^{l f}(\mathcal{A}(U))$. Moreover, following Jacobinski, it is possible to describe the addition law of the group $S K_{0}\left(\mathfrak{B}^{l f}(\mathcal{A}(U))\right)$ solely in terms of operations on elements of $\mathfrak{B}_{1}^{l f}(\mathcal{A}(U))$ (cf. [C, R2] $\$ 49$, especially 49.10-11 and 49.15). However, whilst the group $\mathrm{Cl}^{l f}(\mathcal{A})$ defined above is a natural analogue of each group $S K_{0}\left(\mathfrak{B}^{l f}(\mathcal{A}(U))\right)$, it is not in general possible to describe it solely in terms of rank one $\mathcal{A}$-bundles unless $X$ has dimension one.

We now introduce a useful class of algebras. 
DEFINITION 3.3. We say that a coherent sheaf of algebras $\mathcal{A}$ is of Swan-type if the following condition is satisfied: an object $\mathcal{V}$ of $\mathfrak{B}^{l p}(\mathcal{A})$ belongs to $\mathfrak{B}^{l f}(\mathcal{A})$ if and only if $\mathcal{V}_{\eta}$ is a free $A$-module.

EXAMPLES 3.4. Let $X$ be a curve. A coherent sheaf of algebras $\mathcal{A}$ is of Swantype if it is commutative ([C, R1], Prop. 35.7), or if $\mathcal{A}_{x}$ is a maximal $\mathcal{O}_{x}$-order in $A$ for each $x \in X_{0}$ (ibid., Th. 26.24(iii)), or if $\mathcal{A}=\mathcal{O}[G]$ for a finite group $G$ (cf. ibid., Th. 32.1). Note that the last reference is to a well-known and much used result of Swan, and this motivated our choice of terminology.

LEMMA 3.5. Let $\mathcal{A}$ be a coherent sheaf of algebras on $X$.

(a) The natural homomorphism $K_{0}^{l f}(\mathcal{A}) \rightarrow K_{0}^{l p}(\mathcal{A})$ is injective if $X$ is a curve or if $\mathcal{A}$ is of Swan-type.

(b) Suppose that $\mathcal{A}$ is of Swan-type. Then the natural homomorphism $K_{0}^{l f}(\mathcal{A}) \rightarrow$ $K_{0}^{l p}(\mathcal{A})$ restricts to give an isomorphism $\mathrm{Cl}^{l f}(\mathcal{A}) \simeq \mathrm{Cl}^{l p}(\mathcal{A})$.

Proof. (a) Let $\mathcal{V}_{1}$ and $\mathcal{V}_{2}$ be any objects of $\mathfrak{B}^{l f}(\mathcal{A})$ which have the same image under the natural map $K_{0}^{l f}(\mathcal{A}) \rightarrow K_{0}^{l p}(\mathcal{A})$. Then there are objects $\mathcal{P}_{1}, \mathcal{P}_{2}$, and $\mathcal{P}_{3}$ of $\mathfrak{B}^{l p}(\mathcal{A})$ which fit into short exact sequences

$$
0 \rightarrow \mathcal{P}_{1} \rightarrow \mathcal{P}_{2} \oplus \mathcal{V}_{i} \rightarrow \mathcal{P}_{3} \rightarrow 0
$$

in $\mathfrak{B}^{l p}(\mathcal{A})$ for both $i=1$ and $i=2$ (cf. [C, R2], (38.20)). Since $\mathfrak{B}^{l f}(\mathcal{A})$ is cofinal in $\mathfrak{B}^{l p}(\mathcal{A})$ we may choose objects $\mathcal{P}_{2}^{\prime}$ and $\mathcal{P}_{3}^{\prime}$ of $\mathfrak{B}^{l p}(\mathcal{A})$ such that both $\mathcal{P}_{3}^{\prime} \oplus \mathcal{P}_{3}$ and $\mathcal{P}_{2}^{\prime} \oplus \mathcal{P}_{3}^{\prime} \oplus \mathcal{P}_{2}$ belong to $\mathfrak{B}^{l f}(\mathcal{A})$. Now consider the short exact sequences

$$
0 \rightarrow \mathcal{P}_{2}^{\prime} \oplus \mathcal{P}_{1} \rightarrow\left(\mathcal{P}_{2}^{\prime} \oplus \mathcal{P}_{3}^{\prime} \oplus \mathcal{P}_{2}\right) \oplus \mathcal{V}_{i} \rightarrow \mathcal{P}_{3}^{\prime} \oplus \mathcal{P}_{3} \rightarrow 0
$$

for $i=1,2$. It follows from these sequences that in order to prove that $\mathcal{V}_{1}$ and $\mathcal{V}_{2}$ have the same class in $K_{0}^{l f}(\mathcal{A})$, it suffices to show that $\mathcal{P}_{2}^{\prime} \oplus \mathcal{P}_{1}$ belongs to $\mathfrak{B}^{l f}(\mathcal{A})$.

Now for each $x \in X$, the short exact sequence of stalks associated to (3.1) is split. In particular, by applying the Krull-Schmidt-Azumaya theorem (see [C, R1], Th. 6.12) to the algebra $A$, we deduce that $\left(\mathcal{P}_{2}^{\prime} \oplus \mathcal{P}_{1}\right)_{\eta}$ is a free $A$-module. If $\mathcal{A}$ is of Swan-type, then it follows that $\mathcal{P}_{2}^{\prime} \oplus \mathcal{P}_{1}$ belongs to $\mathfrak{B}^{l f}(\mathcal{A})$, as required. On the other hand, if $X$ is a curve, then each stalk $\mathcal{O}_{x}$ is a discrete valuation ring, and so (loc. cit., Th. 6.12(ii)) can be applied in conjunction with (loc. cit., Prop. 30.17) to deduce that $\left(\mathcal{P}_{2}^{\prime} \oplus \mathcal{P}_{1}\right)_{x}$ is a free $\mathcal{A}_{x}$-module, and hence that $\mathcal{P}_{2}^{\prime} \oplus \mathcal{P}_{1}$ belongs to $\mathfrak{B}^{l f}(\mathcal{A})$.

(b) It is clear that the natural injection $K_{0}^{l f}(\mathcal{A}) \rightarrow K_{0}^{l p}(\mathcal{A})$ restricts to give an injection $\mathrm{Cl}^{l f}(\mathcal{A}) \rightarrow \mathrm{Cl}^{l p}(\mathcal{A})$, and for the remainder of this argument we identify $\mathrm{Cl}^{l f}(\mathcal{A})$ with its image under this injection. We therefore only need to prove that any element in $\mathrm{Cl}^{l p}(\mathcal{A})$ belongs to $\mathrm{Cl}^{l f}(\mathcal{A})$.

Any element $c$ of $K_{0}^{l p}(\mathcal{A})$ has the form $\left(\mathcal{L}_{1}\right)-\left(\mathcal{L}_{2}\right)$ for projective $\mathcal{A}$-bundles $\mathcal{L}_{1}$ and $\mathcal{L}_{2}$. If $c$ belongs to $\mathrm{Cl}^{l p}(\mathcal{A})$, then $\left(\mathcal{L}_{1, \eta}\right)=\left(\mathcal{L}_{2, \eta}\right) \in K_{0}(A)$ and this implies, via ([C, R2], 38.20) and the Krull-Schimdt-Azumaya theorem, that the projective $A$-modules $\mathcal{L}_{1, \eta}$ and $\mathcal{L}_{2, \eta}$ are isomorphic. 
By Lemma 2.6, there exists a projective $\mathcal{A}$-bundle $\mathcal{L}$ such that $\mathcal{L}_{1} \oplus \mathcal{L}$ is an $\mathcal{A}$-bundle. It follows that $\mathcal{L}_{2} \oplus \mathcal{L}$ is a projective $\mathcal{A}$-bundle with generic stalk isomorphic to the free $A$-module $\mathcal{L}_{1, \eta} \oplus \mathcal{L}_{\eta}=\left(\mathcal{L}_{1} \oplus \mathcal{L}\right)_{\eta}$. Since $\mathcal{A}$ is of Swan-type it follows that $\mathcal{L}_{2} \oplus \mathcal{L}$ is an $\mathcal{A}$-bundle. But in $\mathrm{Cl}^{l p}(\mathcal{A})$ one has $c=\left(\mathcal{L}_{1} \oplus \mathcal{L}\right)-\left(\mathcal{L}_{2} \oplus \mathcal{L}\right)$, and hence we deduce that $c \in \mathrm{Cl}^{l f}(\mathcal{A})$.

Remark 3.5.1. (a) Note that the proof of Lemma 3.5(a) actually shows that the natural homomorphism $K_{0}^{l f}(\mathcal{A}) \rightarrow K_{0}^{l p}(\mathcal{A})$ is injective whenever $\mathfrak{B}^{l f}(\mathcal{A})$ is closed under the formation of kernels in $\mathfrak{B}^{l p}(\mathcal{A})$.

(b) At this stage, we have introduced hypotheses of isotriviality and of being of Swan-type on sheaves of algebras $\mathcal{A}$. Some of the key results of this paper actually require neither hypothesis (see for example Theorems 3.11 and 5.2). However, in some other cases, we shall impose the condition of isotriviality in order to facilitate the study of the effect of replacing $\mathcal{A}$ by the sheaf $\mathcal{A}_{s s}$ of semisimple algebras, and to define and study a version of the projective Euler characteristic construction introduced by Chinburg in [Ch]. We shall impose the condition of being of Swan-type in order to allow passage from sheaves of semisimple algebras to sheaves of commutative algebras by means of Lemma 3.5 and arguments involving Morita theory. At the moment, we do not know whether similar reductions can be formulated and studied without imposing such hypotheses.

For any coherent sheaves of algebras $\mathcal{A}$ and $\mathcal{B}$ on $X$ we shall let $\operatorname{Coh}(\mathcal{A}, \mathcal{B})$ denote the category of coherent $\mathcal{O}$-modules which possess actions of $\mathcal{A}$ on the left and of $\mathcal{B}$ on the right which commute in the obvious sense. We shall make use of the following examples.

EXAMPLE 3.6. For each natural number $n$, we let $M_{n}(\mathcal{A})^{\circ}$ denote the sheaf on $X$ for which $M_{n}(\mathcal{A})^{\circ}(U)$ is equal to the opposite ring of the ring of $n \times n$-matrices over $\mathcal{A}(U)$ for each open subset $U$ of $X$. If $\mathcal{P}$ denotes the direct sum of $n$ copies of $\mathcal{A}$, then there is an isomorphism between $M_{n}(\mathcal{A})^{\circ}$ and the sheaf $\mathcal{H o m}_{\mathcal{A}}(\mathcal{P}, \mathcal{P})$ and so $\mathcal{P} \in \operatorname{Coh}\left(\mathcal{A}, M_{n}(\mathcal{A})\right)$.

Another example is the sheaf $\mathcal{Q}:=\mathcal{H o m}_{\mathcal{A}}(\mathcal{P}, \mathcal{A})$ which belongs to $\operatorname{Coh}\left(M_{n}(\mathcal{A}), \mathcal{A}\right)$. For each $\mathcal{V} \in \mathfrak{C} o h\left(M_{n}(\mathcal{A})\right)$ and $\mathcal{W} \in \mathfrak{C o h}(\mathcal{A})$ we may therefore define

$$
\begin{aligned}
G_{\mathcal{P}}(\mathcal{V}) & :=\mathcal{P} \otimes_{M_{n}(\mathcal{A})} \mathcal{V} \in \mathfrak{C o h}(\mathcal{A}), \\
F_{\mathcal{P}}(\mathcal{W}) & :=\mathcal{Q} \otimes_{\mathcal{A}} \mathcal{W} \in \operatorname{Coh}\left(M_{n}(\mathcal{A})\right)
\end{aligned}
$$

We obtain in this way covariant functors

$$
G_{\mathcal{P}}: \operatorname{Coh}\left(M_{n}(\mathcal{A})\right) \rightarrow \operatorname{Coh}(\mathcal{A})
$$

and

$$
F_{\mathcal{P}}: \operatorname{Coh}(\mathcal{A}) \rightarrow \operatorname{Coh}\left(M_{n}(\mathcal{A})\right)
$$


LEMMA 3.7. (a) The functors $G_{\mathcal{P}}$ and $F_{\mathcal{P}}$ are mutually inverse.

(b) Choose $\mathcal{V} \in \mathfrak{C o h}\left(M_{n}(\mathcal{A})\right)$. Then $\mathcal{V} \in \mathfrak{B}^{l p}\left(M_{n}(\mathcal{A})\right)$ if and only if $G_{\mathcal{P}}(\mathcal{V}) \in$ $\mathfrak{B}^{l p}(\mathcal{A})$. If $\mathcal{V} \in \mathfrak{B}^{l f}\left(M_{n}(\mathcal{A})\right)$, then $G_{\mathcal{P}}(\mathcal{V}) \in \mathfrak{B}^{l f}(\mathcal{A})$.

Proof. This will follow by a standard Morita equivalence type argument.

To prove (a) we must show that if $\mathcal{V} \in \mathfrak{C} o h\left(M_{n}(\mathcal{A})\right)$ and $\mathcal{W} \in \mathfrak{C} o h(\mathcal{A})$, then $F_{\mathcal{P}}\left(G_{\mathcal{P}}(\mathcal{V})\right) \cong \mathcal{V}$ in $\operatorname{Coh}\left(M_{n}(\mathcal{A})\right)$ and $G_{\mathcal{P}}\left(F_{\mathcal{P}}(\mathcal{W})\right) \cong \mathcal{W}$ in $\mathfrak{C} o h(\mathcal{A})$. We recall that $F_{\mathcal{P}}\left(G_{\mathcal{P}}(\mathcal{V})\right)$ and $G_{\mathcal{P}}\left(F_{\mathcal{P}}(\mathcal{W})\right)$ are the sheaves which are associated to the presheaves $F_{\mathcal{P}}\left(G_{\mathcal{P}}(\mathcal{V})\right)^{\prime}$ and $G_{\mathcal{P}}\left(F_{\mathcal{P}}(\mathcal{W})\right)^{\prime}$ defined by

$$
F_{\mathcal{P}}\left(G_{\mathcal{P}}(\mathcal{V})\right)^{\prime}(U)=\operatorname{Hom}_{\mathcal{A}(U)}(\mathcal{P}(U), \mathcal{A}(U)) \otimes_{\mathcal{A}(U)}\left(\mathcal{P}(U) \otimes_{M_{n}(\mathcal{A})(U)} \mathcal{V}(U)\right)
$$

and

$$
G_{\mathcal{P}}\left(F_{\mathcal{P}}(\mathcal{W})\right)^{\prime}(U)=\mathcal{P}(U) \otimes_{M_{n}(\mathcal{A})(U)}\left(\operatorname{Hom}_{\mathcal{A}(U)}(\mathcal{P}(U), \mathcal{A}(U)) \otimes_{\mathcal{A}(U)} \mathcal{W}(U)\right)
$$

for each affine open subset $U$ of $X$.

We let $\theta: F_{\mathcal{P}}\left(G_{\mathcal{P}}(\mathcal{V})\right)^{\prime} \rightarrow \mathcal{V}$ and $\psi: G_{\mathcal{P}}\left(F_{\mathcal{P}}(\mathcal{W})\right)^{\prime} \rightarrow \mathcal{W}$ denote the presheaf morphisms which on each affine open subset $U$ of $X$ satisfy

$$
\theta(\lambda \otimes \pi \otimes v)=\lambda(\pi)(v)
$$

and

$$
\psi(\pi \otimes \lambda \otimes w)=\lambda(\pi)(w)
$$

for each $\pi \in \mathcal{P}(U), \lambda \in \operatorname{Hom}_{\mathcal{A}(U)}(\mathcal{P}(U), \mathcal{A}(U)), v \in \mathcal{V}(U)$ and $w \in \mathcal{W}(U)$.

The standard Morita equivalence arguments (cf. [C, R1] (3.54)) imply that $\theta$ and $\psi$ induce isomorphisms on stalks for each $x \in X$. They therefore induce isomorphisms between $F_{\mathcal{P}}\left(G_{\mathcal{P}}(\mathcal{V})\right)$ and $\mathcal{V}$ in $\mathfrak{C o h}\left(M_{n}(\mathcal{A})\right)$ and between $G_{\mathcal{P}}\left(F_{\mathcal{P}}(\mathcal{W})\right)$ and $\mathcal{W}$ in $\operatorname{Coh}(\mathcal{A})$, respectively.

Choose $x \in X$. Then the functor $N \mapsto \mathcal{P}_{x} \otimes_{M_{n}\left(\mathcal{A}_{x}\right)} N$ induces an equivalence between the categories of finitely generated projective $M_{n}\left(\mathcal{A}_{x}\right)$-modules and of finitely generated projective $\mathcal{A}_{x}$-modules, and sends free $M_{n}\left(\mathcal{A}_{x}\right)$-modules to free $\mathcal{A}_{x}$-modules. In conjunction with (a), this proves (b).

PROPOSITION 3.8. Suppose that $\mathcal{A}$ is a coherent sheaf of algebras on X. Let $n$ be a natural number, and let $\mathcal{P}$ denote the direct sum of $n$ copies of $\mathcal{A}$. Then for each integer $i \geqslant 0$, the functor $G_{\mathcal{P}}$ induces a morphism

$$
G_{\mathcal{A}, n}^{i}: K_{i}^{l f}\left(M_{n}(\mathcal{A})\right) \rightarrow K_{i}^{l f}(\mathcal{A}) .
$$

(a) If $X$ is a curve, or $M_{n}(\mathcal{A})$ is of Swan-type, then $G_{\mathcal{A}, n}^{0}$ is injective.

(b) If $\mathcal{A}$ is of Swan-type, then $G_{\mathcal{A}, n}^{0}$ restricts to give an isomorphism

$$
\mathrm{Cl}^{l f}\left(M_{n}(\mathcal{A})\right) \rightarrow \mathrm{Cl}^{l f}(\mathcal{A}) .
$$

Proof. The existence of the morphisms $G_{\mathcal{A}, n}^{i}$ is a direct consequence of Lemma 3.7. 
Now set $\mathcal{B}:=M_{n}(\mathcal{A})$. Then Lemma 3.7 implies that the functors $F_{\mathcal{P}}$ and $G_{\mathcal{P}}$ induce an equivalence of categories between $\mathfrak{B}^{l p}(\mathcal{B})$ and $\mathfrak{B}^{l p}(\mathcal{A})$. Since the higher algebraic $K$-theory functors are invariants of categories, it follows that there are induced isomorphisms

$$
\mu_{i}: K_{i}^{l p}(\mathcal{B}) \stackrel{\sim}{\rightarrow} K_{i}^{l p}(\mathcal{A})
$$

for each $i \geqslant 0$. In particular, there is a commutative diagram

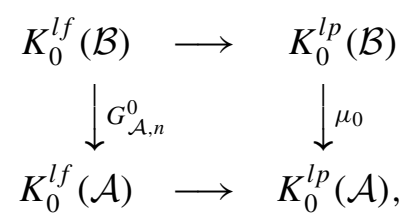

where the two horizontal arrows represent the obvious natural maps. Part (a) thus follows from the fact that if $X$ is a curve, or $\mathcal{B}$ is of Swan-type, then Lemma 3.5(a) implies that the upper horizontal arrow is injective.

To prove part (b), we first observe that if $\mathcal{A}$ is of Swan-type, then so is $\mathcal{B}$. In order to show this, suppose that $\mathcal{V}$ is an object of $\mathfrak{B}^{l p}(\mathcal{B})$ for which $\mathcal{V}_{\eta}$ is a free $\mathcal{B}_{\eta^{-}}$ module. We must show that $\mathcal{V}$ belongs to $\mathfrak{B}^{l f}(\mathcal{B})$. Now $\mathcal{V}_{1}:=G_{\mathcal{P}}(\mathcal{V})$ belongs to $\mathfrak{B}^{l p}(\mathcal{A})$, and $\mathcal{V}_{1, \eta}$ is isomorphic to $\left(\mathcal{P}_{\eta}\right)^{m}$ for some integer $m$. Since $\mathcal{A}$ is assumed to be of Swan-type, it follows that the $\mathcal{A}_{x}$-module $\mathcal{V}_{1, x}$ is isomorphic to $\left(\mathcal{P}_{x}\right)^{m}$ for each $x \in X$. Standard Morita theory now implies that the $\mathcal{B}_{x}$-module $\mathcal{V}_{x}$ is isomorphic to $\left(\mathcal{B}_{x}\right)^{m}$, and since this is true for all $x \in X$, it follows that $\mathcal{V}$ belongs to $\mathfrak{B}^{l p}(\mathcal{B})$, as required.

It now follows from part (a) that $G_{\mathcal{A}, n}^{0}$ is injective. By applying Lemma 3.5(b) to both $\mathcal{A}$ and $\mathcal{B}$, we see that we therefore only need to prove that $G_{\mathcal{A}, n}^{0}\left(\mathrm{Cl}^{l p}(\mathcal{B})\right)=$ $\mathrm{Cl}^{l p}(\mathcal{A})$. This last equality follows easily from Lemma 3.7.

Let $\mathcal{A}$ be an isotrivial sheaf of orders. If $\mathcal{A}_{s s}(X)$ is semisimple and for example $X$ is a curve, then Lemma 1.8(b) and Proposition 3.8(a) together reduce the study of $\mathcal{A}_{s s}$-bundles to the study of bundles over isotrivial sheaves of commutative orders. Our next result further reduces this case to the study of bundles on affine covers of $X$.

For each coherent sheaf of commutative algebras $\mathcal{A}$ on $X$ we let $f_{\mathcal{A}}: \operatorname{Spec}(\mathcal{A}) \rightarrow$ $X$ denote the corresponding affine spectrum (cf. [D, Gr], Chap. $1, \S 9$ ). Note that in general, $\operatorname{Spec}(\mathcal{A})$ is not irreducible. Let $\mathfrak{B}_{n}\left(\mathcal{O}_{\operatorname{Spec}(\mathcal{A})}\right)$ denote the category of $\mathcal{O}_{\operatorname{Spec}(\mathcal{A})}$-modules which are locally free of constant rank $n$. We set

$$
\operatorname{Pic}(\mathcal{A}):=H^{1}\left(X, \mathcal{A}^{*}\right)
$$

thus $\operatorname{Pic}(\mathcal{A})$ is the Picard group of the ringed space $(X, \mathcal{A})$ (cf. [H], p.143).

PROPOSITION 3.9. Let $\mathcal{A}$ be a coherent sheaf of commutative algebras on $X$. Then, for each natural number $n$, the direct image functor $\left(f_{\mathcal{A}}\right)_{*}$ induces an equivalence of categories between $\mathfrak{B}_{n}\left(\mathcal{O}_{\operatorname{Spec}(\mathcal{A})}\right)$ and $\mathfrak{B}_{n}(\mathcal{A})$. If $X$ is a curve, then the 
determinant map $\operatorname{det}_{\mathcal{A}}(-): \mathfrak{B}^{l f}(\mathcal{A}) \rightarrow \mathfrak{B}_{1}(\mathcal{A})$ induces an isomorphism $\mathrm{Cl}^{l f}(\mathcal{A}) \stackrel{\sim}{\rightarrow}$ $\operatorname{Pic}(\mathcal{A})$. In particular, if $\mathcal{V} \in \mathfrak{B}_{1}(\mathcal{A})$ then $(\mathcal{V})=0 \in \mathrm{Cl}^{\text {lf }}(\mathcal{A})$ if and only if $\mathcal{V}$ is isomorphic to $\mathcal{A}$.

Proof. We first note that it is well known that, since $\mathcal{A}$ is a coherent sheaf of algebras on $X$, the bijection of Lemma 2.4 preserves the underlying group structures in the case $n=1$.

Next, we recall that the direct image functor $\left(f_{\mathcal{A}}\right)_{*}$ induces an equivalence of categories between the category of coherent sheaves on $\operatorname{Spec}(\mathcal{A})$ and $\operatorname{Coh}(\mathcal{A})$, and that an inverse to $\left(f_{\mathcal{A}}\right)_{*}$ can be described in the following way. For each object

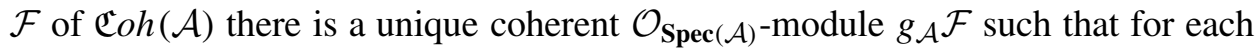
affine open subset $U$ of $X$, the restriction of $g_{\mathcal{A}} \mathcal{F}$ to $f_{\mathcal{A}}^{-1}(U)(\cong \operatorname{Spec}(\mathcal{A}(U)))$ is the sheaf which is associated to the $\mathcal{A}(U)$-module $\mathcal{F}(U)$. Then the functor $g_{\mathcal{A}}$ defined by $\mathcal{F} \mapsto g_{\mathcal{A}} \mathcal{F}$ is inverse to $\left(f_{\mathcal{A}}\right)_{*}$ (cf. [D, Gr], Chap. $\left.1, \S 9\right)$. The following sublemma gives a proof of the first part of Proposition 3.9.

SUBLEMMA 3.9.1. Let $n$ be a natural number. Then $\left(f_{\mathcal{A}}\right)_{*}$ induces an equivalence of categories between $\mathfrak{B}_{n}\left(\mathcal{O}_{\operatorname{Spec}(\mathcal{A})}\right)$ and $\mathfrak{B}_{n}(\mathcal{A})$.

Proof. If $\mathcal{F}$ belongs to $\mathfrak{B}_{n}(\mathcal{A})$, then for each $x \in X_{0}$ there is an affine open neighbourhood $U_{x}$ of $x$ such that $\mathcal{F}\left(U_{x}\right)$ is a free $\mathcal{A}\left(U_{x}\right)$-module of rank $n$. This in turn implies that $g_{\mathcal{A}} \mathcal{F}$ is a vector bundle on $\operatorname{Spec}(\mathcal{A})$ of constant rank $n$, since $\left\{f_{\mathcal{A}}^{-1}\left(U_{x}\right): x \in X_{0}\right\}$ is an affine open covering of $\operatorname{Spec}(\mathcal{A})$ and, for each $x \in X_{0}$, the restriction of $g_{\mathcal{A}} \mathcal{F}$ to $f_{\mathcal{A}}^{-1}\left(U_{x}\right)$ is the sheaf which is associated to $\mathcal{F}\left(U_{x}\right)$.

We must also show that if $\mathcal{V}$ is any bundle on $\operatorname{Spec}(\mathcal{A})$ which has rank $n$, then $\left(f_{\mathcal{A}}\right)_{*} \mathcal{V}$ belongs to $\mathfrak{B}_{n}(\mathcal{A})$. Now, for each $x \in X_{0}$ there is a natural isomorphism

$$
\left(\left(f_{\mathcal{A}}\right)_{*} \mathcal{V}\right)_{x} \cong \bigoplus_{y \in f_{\mathcal{A}}^{-1}(x),} \mathcal{V}_{y}
$$

of $\mathcal{A}_{x}$-modules (cf. for example, [H], Chap. 3, Cor. 12.9). Thus, since $\left(f_{\mathcal{A}}\right)_{*}$ $\mathcal{O}_{\operatorname{Spec}(\mathcal{A})} \cong \mathcal{A}$ this implies that $\left(\left(f_{\mathcal{A}}\right)_{*} \mathcal{V}\right)_{x}$ is indeed a free $\mathcal{A}_{x}$-module of rank $n$.

We now restrict ourselves to the case in which $X$ is a curve. In this case each connected component of $\operatorname{Spec}(\mathcal{A})$ is Noetherian, of dimension one and possesses ample line bundles and so there is an isomorphism

$$
K_{0}(\operatorname{Spec}(\mathcal{A})) \cong \mathbb{Z}^{\pi_{0}(\operatorname{Spec}(\mathcal{A}))} \oplus \operatorname{Pic}(\operatorname{Spec}(\mathcal{A}))
$$

given by

$$
(\mathcal{V}) \mapsto\left(\operatorname{rank}(\mathcal{V}),\left(\operatorname{det}_{\mathcal{O}_{\text {Spec }(\mathcal{A})}} \mathcal{V}\right)\right)
$$

(cf. [Fu, L], Chap. V, Cor. 3.10 and Chap. III, Th. 1.7). We let $\left(g_{\mathcal{A}}\right)_{*}$ denote the homomorphism $K_{0}^{l f}(\mathcal{A}) \rightarrow K_{0}(\operatorname{Spec}(\mathcal{A}))$ which is induced by the functor $g_{\mathcal{A}}$. 
SUBLEMMA 3.9.2. Suppose that $X$ is a curve. Then the composite map

$$
K_{0}^{l f}(\mathcal{A}) \stackrel{\left(g_{\mathcal{A}}\right)_{*}}{\rightarrow} K_{0}(\operatorname{Spec}(\mathcal{A})) \stackrel{\sim}{\rightarrow} \mathbb{Z}^{\pi_{0}(\operatorname{Spec}(\mathcal{A}))} \oplus \operatorname{Pic}(\operatorname{Spec}(\mathcal{A}))
$$

is injective and has image $\mathbb{Z} \oplus \operatorname{Pic}(\operatorname{Spec}(\mathcal{A}))$.

Proof. The only point which requires an argument is the claim that $\left(g_{\mathcal{A}}\right)_{*}$ is injective. To show this, we use the fact that for any locally free sheaf $\mathcal{F}$ on $\operatorname{Spec}(\mathcal{A})$ there is a locally free sheaf $\mathcal{F}^{\prime}$ on $\operatorname{Spec}(\mathcal{A})$ such that $\mathcal{F} \oplus \mathcal{F}^{\prime}$ has constant rank.

Suppose that $\mathcal{F}_{1}$ and $\mathcal{F}_{2}$ are $\mathcal{A}$-bundles with $\left(g_{\mathcal{A}} \mathcal{F}_{1}\right)=\left(g_{\mathcal{A}} \mathcal{F}_{2}\right) \in K_{0}(\operatorname{Spec}(\mathcal{A}))$. Then there are locally free sheaves $\mathcal{P}_{1}, \mathcal{P}_{2}$ and $\mathcal{P}_{3}$ on $\operatorname{Spec}(\mathcal{A})$ and short exact sequences of $\mathcal{O}_{\operatorname{Spec}(\mathcal{A}) \text {-modules }}$

$$
0 \rightarrow \mathcal{P}_{1} \rightarrow \mathcal{P}_{2} \oplus g_{\mathcal{A}} \mathcal{F}_{i} \rightarrow \mathcal{P}_{3} \rightarrow 0
$$

for both $i=1$ and $i=2$ (cf. [C, R2], (38.20)). Choosing locally free sheaves $\mathcal{P}_{3}^{\prime}$ and $\mathcal{P}_{2}^{\prime}$ on $\operatorname{Spec}(\mathcal{A})$ such that $\mathcal{P}_{3}^{\prime} \oplus \mathcal{P}_{3}$ and $\mathcal{P}_{2}^{\prime} \oplus \mathcal{P}_{3}^{\prime} \oplus \mathcal{P}_{2}$ both have constant rank, there are therefore short exact sequences of $\mathcal{O}_{\operatorname{Spec}(\mathcal{A}) \text {-modules }}$

$$
0 \rightarrow \mathcal{P}_{2}^{\prime} \oplus \mathcal{P}_{1} \rightarrow\left(\mathcal{P}_{2}^{\prime} \oplus \mathcal{P}_{3}^{\prime} \oplus \mathcal{P}_{2}\right) \oplus g_{\mathcal{A}} \mathcal{F}_{i} \rightarrow \mathcal{P}_{3}^{\prime} \oplus \mathcal{P}_{3} \rightarrow 0
$$

for both $i=1$ and $i=2$ in which all terms are locally free of constant rank. Applying $\left(f_{\mathcal{A}}\right)_{*}$ to these sequences (and using Sublemma 3.9.1) gives two short exact sequences of $\mathcal{A}$-bundles which together imply that $\left(\mathcal{F}_{1}\right)=\left(\mathcal{F}_{2}\right) \in K_{0}^{l f}(\mathcal{A})$.

The injective map of Sublemma 3.9.2 restricts to give an isomorphism $\mathrm{Cl}^{l f}$ $(\mathcal{A}) \simeq \operatorname{Pic}(\operatorname{Spec}(\mathcal{A}))$, and so one need only show that the $\operatorname{bijection} \operatorname{Pic}(\mathcal{A}) \stackrel{\sim}{\rightarrow}$ $\operatorname{Pic}(\operatorname{Spec}(\mathcal{A}))$ which is induced by the functor $g_{\mathcal{A}}$ respects the underlying group structures (i.e. that it commutes with taking tensor products). This is in turn an easy exercise which we leave to the reader.

This completes the proof of Proposition 3.9.

To end this section, we describe a version of Quillen's localisation theorem which is valid in the case that $X$ is a curve. We then describe an easy consequence of this theorem which will be very important in subsequent sections.

In the remainder of this section $X$ is a curve, and $\mathcal{A}$ is a sheaf of orders on $X$. Let $x \in X_{0}$. We write $\mathcal{O}_{\hat{x}}$ for the completion of the discrete valuation ring $\mathcal{O}_{x}$. Since $x$ is a specialisation of $\eta$, there is a natural map from $\mathcal{O}_{x}$ to $K$, and we set $K_{\hat{x}}:=\mathcal{O}_{\hat{x}} \otimes_{\mathcal{O}_{x}} K$. For any $\mathcal{O}$-module $\mathcal{V}$ we set $\mathcal{V}_{\hat{x}}:=\mathcal{O}_{\hat{x}} \otimes_{\mathcal{O}_{x}} \mathcal{V}_{x}$. We recall that, for each $\mathcal{A}$-module $\mathcal{V}$, the stalk $\mathcal{V}_{x}$ is a free $\mathcal{A}_{x}$-module if and only if $\mathcal{V}_{\hat{x}}$ is a free $\mathcal{A}_{\hat{x}}$-module (cf. [C, R1], Prop. 30.17). We set $A_{\hat{x}}=K_{\hat{x}} \otimes_{\mathcal{O}_{\hat{x}}} \mathcal{A}_{\hat{x}}$.

DEFINITION 3.10. Suppose that $X$ is a curve.

(a) For each $x \in X_{0}$, we let $H\left(\mathcal{A}_{\hat{x}}\right)$ denote the category of $\mathcal{A}_{\hat{x}}$-modules which are finite and have resolutions of length 1 by finitely generated free $\mathcal{A}_{\hat{x}}$-modules. 
Then it follows from the Horseshoe Lemma of homological algebra (see for instance [C, R2], (38.39)) that $H\left(\mathcal{A}_{\hat{x}}\right)$ is an exact category.

(b) Write $j_{X}: \operatorname{Spec}(K) \rightarrow X$ for the natural inclusion morphism. Let $\mathfrak{H}(\mathcal{A})$ denote the category of $\mathcal{A}$-modules $\mathcal{F}$ on $X$ which are coherent as $\mathcal{O}$-modules and are such that:

(i) $j_{X}^{*} \mathcal{F}=0$, and

(ii) There is a short exact sequence of $\mathcal{A}$-modules

$$
0 \rightarrow \mathcal{P}^{-1} \rightarrow \mathcal{P}^{0} \rightarrow \mathcal{F} \rightarrow 0
$$

in which both $\mathcal{P}^{-1}$ and $\mathcal{P}^{0}$ belong to $\mathfrak{B}^{l f}(\mathcal{A})$.

By combining Lemma 2.5 with Schanuel's lemma, the Krull-Schmidt-Azumaya theorem and the exactness of $H\left(\mathcal{A}_{\hat{x}}\right)$ for each $x \in X_{0}$, it may be shown that $\mathfrak{H}(\mathcal{A})$ is an exact category (cf. for example [Sr], p. 194).

THEOREM 3.11 (Quillen). Let $X$ be a curve, $\mathcal{A}$ a sheaf of orders on $X$ and $q a$ non-negative integer.

(a) There is a long exact sequence of groups

$$
K_{q+1}(A) \rightarrow K_{q}(\mathfrak{H}(\mathcal{A})) \rightarrow K_{q}^{l f}(\mathcal{A}) \rightarrow K_{q}(A) .
$$

(b) For each point $x \in X_{0}$, there is a long exact sequence of groups

$$
K_{q+1}\left(A_{\hat{x}}\right) \rightarrow K_{q}\left(H\left(\mathcal{A}_{\hat{x}}\right)\right) \rightarrow K_{q}^{f}\left(\mathcal{A}_{\hat{x}}\right) \rightarrow K_{q}\left(A_{\hat{x}}\right) .
$$

We are very grateful to D. Grayson for pointing out that the proof of this theorem requires only a minor modification of the proof of the localisation theorem for projective modules as presented in ( [Gra], The localization theorem for projective modules). For the reader's convenience we shall give a few remarks comparing the proofs of Theorem 3.11(a) and of the localization theorem in (ibid). Similar remarks apply mutatis mutandis to the proof of Theorem 3.11(b).

In comparison to the statement and proof of the localization theorem in (ibid) we shall make the following notational changes:

- $U$ is replaced by $\operatorname{Spec}(K)$ and $j$ by the morphism $j_{X}$;

- $H$ is replaced by the category $\mathfrak{H}(\mathcal{A})$;

- $P$ is replaced by the category $\mathfrak{B}^{l f}(\mathcal{A})$;

- $V$ is replaced by the category $\mathfrak{B}^{p}(A)$;

- $P_{1}$ is replaced by the category of $\mathcal{A}$-modules which have a resolution of length 1 by $\mathcal{A}$-bundles.

With these notational changes the argument of ([Gra], the localization theorem for projective modules) is formally the same up to Lemma 2 (ibid). In the new context this lemma is replaced by the following result.

LEMMA 3.12. If $\mathcal{C}$ is an $\mathcal{A}$-bundle on $X$, then $\mathcal{C} \subseteq\left(j_{X}\right)_{*}\left(j_{X}^{*}\right) \mathcal{C}$. Furthermore, if $\mathcal{L}$ is any locally finitely generated $\mathcal{A}$-submodule of $\left(j_{X}\right)_{*}\left(j_{X}^{*}\right) \mathcal{C}$ then there is an ideal sheaf $\mathcal{I}$ on $X$ and an integer $m$ such that $\mathcal{L} \subseteq \mathcal{I}^{m} \otimes_{\mathcal{O}} \mathcal{C} \subseteq\left(j_{X}\right)_{*}\left(j_{X}^{*}\right) \mathcal{C}$. 
Proof. The first inclusion is clear since $\left(j_{X}\right)_{*}\left(j_{X}^{*}\right) \mathcal{C}$ is equal to the constant sheaf on $X$ defined by the $A$-module $K \otimes_{\mathcal{O}(U)} \mathcal{C}(U)$ (for any affine open subset $U$ of $X)$. To prove the assertion concerning $\mathcal{L}$ we take a finite covering $\mathcal{U}=\left\{U_{i}\right\}$ of $X$ by affine open subsets such that $\mathcal{L}\left(U_{i}\right)$ is a finitely generated $\mathcal{O}\left(U_{i}\right)$-module for each $i$. For each index $i$ there is a fractional ideal $I\left(U_{i}\right)$ of $\mathcal{O}\left(U_{i}\right)$ such that $\mathcal{L}\left(U_{i}\right) \subseteq I\left(U_{i}\right) \otimes_{\mathcal{O}\left(U_{i}\right)} \mathcal{C}\left(U_{i}\right)$. Since $X$ is a curve, the set $Z:=\left\{x \in X_{0}: x \in U_{i}\right.$ and $I\left(U_{i}\right)_{x} \neq \mathcal{O}_{x}$ for some index $\left.i\right\}$ is finite. Let $\mathcal{I}$ denote the sheaf of ideals on $X$ corresponding to the closed subscheme $Z \subseteq X$. Then $\mathcal{L} \subseteq \mathcal{I}^{-n} \otimes_{\mathcal{O}} \mathcal{C}$ for any sufficiently large integer $n$.

The proof of Lemma 3 (ibid) now proceeds unchanged until the introduction of the ordered set $L a t$. In this new context, if $\mathcal{B}$ is an $\mathcal{A}$-bundle on $X, W$ is the $A$ module corresponding to $j_{X}^{*} \mathcal{B}$ and $Z$ is a free $A$-module equipped with a surjective map of $A$-modules $Z \rightarrow W$, then $\underline{L a t}$ is the set of $\mathcal{A}$-bundles $\mathcal{L}$ on $X$ such that

(1) $\mathcal{L} \subset\left(j_{X}\right)_{*} Z$,

(2) $j_{X}^{*} \mathcal{L}=Z$,

(3) $\mathcal{B}$ is the image of composite map $\mathcal{L} \subset\left(j_{X}\right)_{*} Z \rightarrow\left(j_{X}\right)_{*} W=\left(j_{X}\right)_{*}\left(j_{X}^{*}\right) \mathcal{B}$.

If $Y$ is the kernel of the surjection $Z \rightarrow W$, then the short exact sequence of $A$-modules

$$
0 \rightarrow Y \rightarrow Z \rightarrow W \rightarrow 0
$$

splits. Since $W$ and $Z$ are free $A$-modules it follows (e.g. via the Krull-SchmidtAzumaya theorem) that $Y$ is a free $A$-module and hence that $Y=j_{X}^{*} \mathcal{C}$ for some $\mathcal{A}$-bundle $\mathcal{C}$. By choosing a splitting of the above short exact sequence, we may identify $Z$ with $j_{X}^{*}(\mathcal{C} \oplus \mathcal{B})$, and via this identification $\mathcal{C} \oplus \mathcal{B}$ belongs to $\underline{\text { Lat }}$. If $\mathcal{L} \subset\left(j_{X}\right)_{*} Z$ is any other element of $\underline{L a t}$ then condition 3) implies that $\underline{\mathcal{L} \subset}$ $\left(j_{X}\right)_{*}\left(j_{X}^{*}\right) \mathcal{C} \oplus \mathcal{B}$. Since $\mathcal{L}$ is locally finitely generated, Lemma 3.12 implies that $\mathcal{L} \subseteq\left(\mathcal{I}^{m} \otimes_{\mathcal{O}} \mathcal{C}\right) \oplus \mathcal{B}$ for some integer $m$. Since for any ideal sheaf $\mathcal{I}$ and integer $m$, the direct sum $\left(\mathcal{I}^{m} \otimes_{\mathcal{O}} \mathcal{C}\right) \oplus \mathcal{B}$ belongs to $\underline{\text { Lat }}$, it follows that $\underline{\text { Lat }}$ is filtering, and this proves the required analogue of Lemma 3 (ibid).

The proof of Theorem 3.11(a) now proceeds exactly as does the proof of the localization theorem for projective modules of (ibid) following Lemma 3 (ibid).

The morphism $K_{0}^{l f}(\mathcal{A}) \rightarrow K_{0}(A)$ which occurs in the exact sequence of Theorem 3.11(a) factors through the map $K_{0}^{l f}(\mathcal{A}) \rightarrow K_{0}^{f}(A)$, and hence this sequence implies that there is an exact sequence

$$
K_{1}^{l f}(\mathcal{A}) \rightarrow K_{1}(A) \rightarrow K_{0}(\mathfrak{H}(\mathcal{A})) \rightarrow \mathrm{Cl}^{l f}(\mathcal{A}) \rightarrow 0 .
$$

We conclude this section by noting that there is an exact sequence

$$
K_{1}\left(\mathcal{A}_{\hat{x}}\right) \rightarrow K_{1}\left(A_{\hat{x}}\right) \rightarrow K_{0}\left(H\left(\mathcal{A}_{\hat{x}}\right)\right) \rightarrow 0 .
$$


This is the local analogue of (3.2), and it follows from Theorem 3.11(b), together with the fact that for each $x \in X_{0}$ the natural maps $K_{1}^{f}\left(\mathcal{A}_{\hat{x}}\right) \rightarrow K_{1}\left(\mathcal{A}_{\hat{x}}\right)$ and $K_{0}^{f}\left(\mathcal{A}_{\hat{x}}\right) \rightarrow K_{0}\left(A_{\hat{x}}\right)$ are bijective and injective, respectively.

The exact sequences (3.2) and (3.3) will be of great importance in Section 5.

\section{Grothendieck Groups of Bundles}

In the remainder of this paper we shall assume, unless explicitly stated otherwise, that $\mathcal{A}$ is a sheaf of orders on $X$. Our primary aim is to study the Grothendieck group $K_{0}^{l f}(\mathcal{A})$.

All cohomology groups will be with respect the Zariski topology, unless stated otherwise.

\section{FILTRATIONS}

In this section we shall define and investigate a natural filtration on $K_{0}^{l f}(\mathcal{A})$.

For each integer $i \geqslant 0$ we let

$$
\sigma_{\mathcal{A}}^{i}: K_{0}^{l f}(\mathcal{A}) \rightarrow K_{0}^{l f}\left(\mathcal{A}_{s s}^{(i)}\right)
$$

denote the morphism which is induced by the functor

$$
\mathcal{A}_{s s}^{(i)} \otimes_{\mathcal{A}}-: \mathfrak{B}^{l f}(\mathcal{A}) \rightarrow \mathfrak{B}^{l f}\left(\mathcal{A}_{s s}^{(i)}\right)
$$

(cf. Lemma 2.3(a)), and we set

$$
D_{i}^{s s}(\mathcal{A}):=\operatorname{Ker}\left(\sigma_{\mathcal{A}}^{i}\right) .
$$

We remind the reader that, by convention, $\mathcal{A}_{s s}^{(0)}$ is the zero sheaf. We write $\sigma_{\mathcal{A}}$ and $D^{s s}(\mathcal{A})$ in place of $\sigma_{\mathcal{A}}^{1}$ and $D_{1}^{s s}(\mathcal{A})$ and we call them the semisimplification homomorphism and the semisimplification kernel of $\mathcal{A}$, respectively.

Let $e$ denote the smallest non-negative integer such that $\mathcal{R}(\mathcal{A})^{e}$ is zero (see Lemma 1.4(c)).

LEMMA 4.1. We have $D_{i}^{s s}(\mathcal{A}) \supseteq D_{i+1}^{s s}(\mathcal{A})$ for each integer $i \geqslant 0$. Also $D_{0}^{s s}(\mathcal{A})=$ $K_{0}^{l f}(\mathcal{A}), D_{1}^{s s}(\mathcal{A}) \subseteq \mathrm{Cl}^{l f}(\mathcal{A})$ and $D_{i}^{s s}(\mathcal{A})=\{0\}$ for each integer $i \geqslant e$.

Proof. The only point which needs an argument is the inclusion $D_{1}^{s s}(\mathcal{A}) \subseteq$ $\mathrm{Cl}^{l f}(\mathcal{A})$. Let $A$ denote the generic stalk of $\mathcal{A}$ and write $A_{s s}$ for the quotient of $A$ by the generic stalk of $\mathcal{R}(\mathcal{A})$. Consider the following diagram:

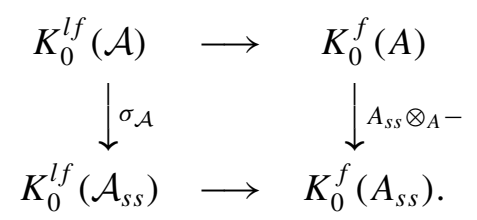


In this diagram the horizontal maps are induced by taking generic stalks. Since the right-hand vertical map is bijective and the diagram is commutative, it follows immediately that $D_{1}^{s s}(\mathcal{A}) \subseteq \mathrm{Cl}^{l f}(\mathcal{A})$.

We shall find the following lemma to be useful when we investigate the filtration of $K_{0}^{l f}(\mathcal{A})$ given by $\left\{D_{i}^{s s}(\mathcal{A}): i \geqslant 0\right\}$. For each pair of natural numbers $a$ and $b$ with $a \geqslant b$ we let $\mathcal{Q}(b, a)$ denote the Zariski sheaf which is associated to the (multiplicative) presheaf $U \mapsto\left(1+\mathcal{R}(U)^{b}\right) /\left(1+\mathcal{R}(U)^{a}\right)$ for each affine open subset $U$ of $X$.

LEMMA 4.2. For each natural number a there is a long exact sequence of pointed sets:

$$
\begin{aligned}
0 \rightarrow & H^{0}\left(X, 1+\mathcal{R}^{a}\right) \rightarrow H^{0}\left(X, \mathcal{A}^{*}\right) \rightarrow H^{0}\left(X,\left(\mathcal{A}_{s s}^{(a)}\right)^{*}\right) \rightarrow \\
& H^{1}\left(X, 1+\mathcal{R}^{a}\right) \stackrel{\alpha}{\rightarrow} H^{1}\left(X, \mathcal{A}^{*}\right) \rightarrow H^{1}\left(X,\left(\mathcal{A}_{s s}^{(a)}\right)^{*}\right) .
\end{aligned}
$$

In addition, for all natural numbers $a, b$ with $a \geqslant b$ there is a long exact sequence of pointed sets:

$$
\begin{aligned}
0 \rightarrow & H^{0}(X, \mathcal{Q}(a, a+1)) \rightarrow H^{0}(X, \mathcal{Q}(b, a+1)) \rightarrow H^{0}(X, \mathcal{Q}(b, a)) \rightarrow \\
& H^{1}(X, \mathcal{Q}(a, a+1)) \stackrel{\beta}{\rightarrow} H^{1}(X, \mathcal{Q}(b, a+1)) \rightarrow H^{1}(X, \mathcal{Q}(b, a)) \rightarrow \\
& H^{2}(X, \mathcal{Q}(a, a+1)) .
\end{aligned}
$$

If $\mathcal{A}$ is isotrivial, then the maps $\alpha$ and $\beta$ are injective.

Proof. Since the sheaf $\mathcal{R}$ is nilpotent, there is a short exact sequence of sheaves

$$
0 \rightarrow 1+\mathcal{R}^{a} \stackrel{\subset}{\rightarrow} \mathcal{A}^{*} \rightarrow\left(\mathcal{A}_{s s}^{(a)}\right)^{*} \rightarrow 0
$$

for each $a \geqslant 1$. The long exact sequence (4.1) is a consequence of applying the result of ([Gi], Chap. IV, 4.2.10) to this sequence. In a similar way, the long exact sequence (4.2) is a consequence of applying ([Gi], Chap. IV, 4.2.10) to the short exact sequence

$$
0 \rightarrow \mathcal{Q}(a, a+1) \stackrel{\complement}{\rightarrow} \mathcal{Q}(b, a+1) \rightarrow \mathcal{Q}(b, a) \rightarrow 0,
$$

and noting that $\mathcal{Q}(a, a+1)$ is central in $\mathcal{Q}(b, a+1)$.

Suppose now that $\mathcal{A}$ is isotrivial, and for brevity, set $J:=\operatorname{Jac}(\mathcal{A}(X))$. Then $H^{0}\left(X, \mathcal{A}^{*}\right)=\mathcal{A}(X)^{*}$, and $H^{0}\left(X,\left(\mathcal{A}_{s s}^{(a)}\right)^{*}\right)=\left(\mathcal{A}(X) / J^{a}\right)^{*}$. Since $J^{a}$ is nilpotent it follows that the natural quotient map $H^{0}\left(X, \mathcal{A}^{*}\right) \rightarrow H^{0}\left(X,\left(\mathcal{A}_{s s}^{(a)}\right)^{*}\right)$ is surjective, and using the exactness of (4.1), this implies that the map $\alpha$ is injective.

In a similar way, the exactness of (4.2) implies that $\beta$ is injective if the map $H^{0}(X, \mathcal{Q}(b, a+1)) \rightarrow H^{0}(X, \mathcal{Q}(b, a))$ is surjective. This in turn will follow if we show that the natural map

$$
\left(1+J^{c}\right) /\left(1+J^{d}\right) \rightarrow H^{0}(X, \mathcal{Q}(c, d))
$$


is bijective for each pair of natural numbers $c, d$ with $c \leqslant d$. We shall therefore prove that such an isomorphism holds.

The claim is trivial if $c=d$, and we prove the result for $c<d$ by an induction on $d-c$. If $d-c=1$, then we have

$$
\mathcal{Q}(c, c+1) \stackrel{\sim}{\rightarrow} \mathcal{R}^{c} / \mathcal{R}^{c+1} \stackrel{\sim}{\rightarrow} \mathcal{O} \otimes_{k} \frac{J^{c}}{J^{c+1}},
$$

where the first isomorphism is induced by mapping $1+r$ to $r$ for each $r \in \mathcal{R}(U)^{c}$ and each open affine subset $U$ of $X$. Hence $H^{0}(X, \mathcal{Q}(c, c+1)) \cong\left(1+J^{c}\right) /(1+$ $\left.J^{c+1}\right)$, as required.

For the inductive step, we consider the following commutative diagram:

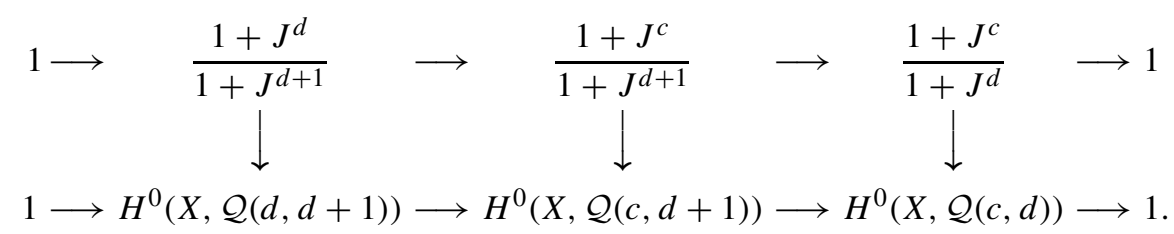

In this diagram the first and third vertical arrows are bijective by the inductive hypothesis, the top row is exact and the bottom row is exact except possibly at $H^{0}(X, \mathcal{Q}(c, d))$. An easy diagram chase shows that the lower row must be exact, and the bijectivity of the second vertical arrow is then obvious. This immediately implies the inductive step.

Remark 4.3. Set $J:=\operatorname{Jac}(\mathcal{A}(X))$. If $\mathcal{R}=\mathcal{O} \otimes_{k} J$ then, for any integer $a \geqslant 0$, there is a natural isomorphism of $\mathcal{O}$-modules

$$
\mathcal{R}^{a} / \mathcal{R}^{a+1} \stackrel{\sim}{\rightarrow} \mathcal{O} \otimes_{k}\left[J^{a} / J^{a+1}\right] .
$$

Since $\mathcal{Q}(a, a+1) \simeq \mathcal{R}^{a} / \mathcal{R}^{a+1}$ (see the proof of Lemma 4.2 above), we therefore have

$$
H^{1}(X, \mathcal{Q}(a, a+1)) \cong H^{1}(X, \mathcal{O}) \otimes_{k}\left[J^{a} / J^{a+1}\right] .
$$

This implies that each module $H^{1}(X, \mathcal{Q}(a, a+1))$ is a 'generalised tangent space' in the sense of Mumford (see the appendix to Lecture 4 of $[M]$ ).

We let $\mathcal{U}^{a f f}(X)$ denote the collection of affine open subsets of $X$ and we set $\mathcal{U}(X):=\mathcal{U}^{a f f}(X) \cup\{X\}$. For each $U \in \mathcal{U}(X)$ we write $K_{0}(\mathcal{A}(U))$ for the Grothendieck group of the category $\mathfrak{B}^{p}(\mathcal{A}(U))$ of finitely generated projective $\mathcal{A}(U)$ modules. If $U \in \mathcal{U}^{a f f}(X)$, then the section functor $H^{0}(U,-): \mathfrak{B}^{l f}(\mathcal{A}) \rightarrow$ $\mathfrak{B}^{p}(\mathcal{A}(U))$ is exact and hence induces a homomorphism $\chi R \Gamma_{\mathcal{A}, U}: K_{0}^{l f}(\mathcal{A}) \rightarrow$ $K_{0}(\mathcal{A}(U))$. In the case $U=X$ we use a projective Euler characteristic homomorphism $\chi R \Gamma_{\mathcal{A}, X}$ which is due to T. Chinburg and which is described in the next proposition.

For any ring $S$ we write $\mathfrak{D}^{b}(S)$ for the derived category of the homotopy category of bounded complexes of $S$-modules. Recall that an object of $\mathfrak{D}^{b}(S)$ is said 
to be perfect if it is isomorphic in $\mathfrak{D}^{b}(S)$ to a bounded complex of finitely generated, projective $S$-modules. We write $\mathfrak{D}^{p}(S)$ for the full triangulated subcategory of $\mathfrak{D}^{b}(S)$ which consists of those complexes which are perfect.

PROPOSITION 4.4 (Chinburg, [Ch], [Ch, E]). (a) If $\mathcal{A}$ is isotrivial and $\mathcal{V}$ is an $\mathcal{A}$ bundle, then the complex $R \Gamma(X, \mathcal{V})$ belongs to $\mathfrak{D}^{p}(\mathcal{A}(X))$. In particular, for each $\mathcal{A}$-bundle $\mathcal{V}$ one can define a projective Euler characteristic

$$
\chi R \Gamma_{\mathcal{A}, X}(\mathcal{V}):=\chi_{\mathcal{A}(X)}(R \Gamma(X, \mathcal{V})) \in K_{0}(\mathcal{A}(X)) .
$$

(b) The association $\mathcal{V} \mapsto \chi R \Gamma_{\mathcal{A}, X}(\mathcal{V})$ induces a homomorphism

$$
\chi R \Gamma_{\mathcal{A}, X}: K_{0}^{l f}(\mathcal{A}) \longrightarrow K_{0}(\mathcal{A}(X)) .
$$

Proof. We set $\Lambda:=\mathcal{A}(X)$, and we let $\mathcal{U}=\left\{U_{i}\right\}$ be a finite affine open covering of $X$. Since $X$ is Noetherian and separated, and $\mathcal{V}$ is a coherent $\mathcal{O}$-module, $R \Gamma(X, \mathcal{V})$ is isomorphic in $\mathfrak{D}^{b}(\Lambda)$ to the Čech complex $C^{\bullet}(\mathcal{U}, \mathcal{V})$ (cf. [H], Chap. III, Lem. 4.4 and Th. 4.5). In addition, since $\mathcal{V}$ is an $\mathcal{A}$-bundle, the sections of $\mathcal{V}$ over an affine open subset $U$ of $X$ are projective over $\mathcal{A}(U)=\mathcal{O}(U) \otimes_{k} \Lambda$ and hence, a fortiori, also over $\Lambda$. Since $X$ is separated, this implies that $C^{\bullet}(\mathcal{U}, \mathcal{V})$ is a bounded complex of projective $\Lambda$-modules. Since, in addition, the cohomology modules of $C^{\bullet}(\mathcal{U}, \mathcal{V})$ are all finitely generated over $\Lambda$ (because $X$ is projective and $\mathcal{V}$ is coherent) it follows by a standard argument that $C^{\bullet}(\mathcal{U}, \mathcal{V})$ belongs to $\mathfrak{D}^{p}(\Lambda)$. In particular, the $\Lambda$-equivariant Euler characteristic of $C^{\bullet}(\mathcal{U}, \mathcal{V})$ is well defined and belongs to $K_{0}(\Lambda)$, and this proves (a).

To prove (b) we must show that if

$$
0 \longrightarrow \mathcal{V}_{1} \longrightarrow \mathcal{V}_{2} \longrightarrow \mathcal{V}_{3} \longrightarrow 0
$$

is any short exact sequence in $\mathfrak{B}^{l f}(\mathcal{A})$, then

$$
\chi_{\Lambda}\left(R \Gamma\left(X, \mathcal{V}_{1}\right)\right)-\chi_{\Lambda}\left(R \Gamma\left(X, \mathcal{V}_{2}\right)\right)+\chi_{\Lambda}\left(R \Gamma\left(X, \mathcal{V}_{3}\right)\right)=0 \in K_{0}(\Lambda)
$$

Since the exactness of (4.5) is preserved if one takes sections over any affine open subset $U$ of $X$, one has a short exact sequence of bounded complexes of $\Lambda$ modules

$$
0 \longrightarrow C^{\bullet}\left(\mathcal{U}, \mathcal{V}_{1}\right) \longrightarrow C^{\bullet}\left(\mathcal{U}, \mathcal{V}_{2}\right) \longrightarrow C^{\bullet}\left(\mathcal{U}, \mathcal{V}_{3}\right) \longrightarrow 0
$$

The above argument shows that this exact sequence gives rise to a distinguished triangle in $\mathfrak{D}^{p}(\Lambda)$

$$
R \Gamma\left(X, \mathcal{V}_{1}\right) \longrightarrow R \Gamma\left(X, \mathcal{V}_{2}\right) \longrightarrow R \Gamma\left(X, \mathcal{V}_{3}\right),
$$

and the equality (4.6) follows by taking Euler characteristics of this triangle.

DEFINITION 4.5. Suppose that $\mathcal{A}$ is an isotrivial sheaf of orders. We define the section functor homomorphism $\gamma_{\mathcal{A}}$ of $\mathcal{A}$ to be the product map

$$
\gamma_{\mathcal{A}}:=\prod_{U \in \mathcal{U}(X)} \chi R \Gamma_{\mathcal{A}, U}: K_{0}^{l f}(\mathcal{A}) \rightarrow \prod_{U \in \mathcal{U}(X)} K_{0}(\mathcal{A}(U)),
$$


and we define the section functor kernel $D^{s f}(\mathcal{A})$ of $\mathcal{A}$ to be the kernel of $\gamma_{\mathcal{A}}$.

We view the subgroup $D^{s f}(\mathcal{A})$ as being a convenient measure of that portion of $K_{0}^{l f}(\mathcal{A})$ which cannot be detected by sectional data.

Remark 4.5.1. Let $f: Y \rightarrow X$ be a finite cover of smooth projective curves over $k$, and suppose that the associated extension $k(Y) / k(X)$ of function fields is a tamely ramified Galois extension with Galois group $G$. Then $f_{*} \mathcal{O}_{Y}$ is an $\mathcal{O}_{X}[G]$ bundle, and $\chi_{k[G]}\left(R \Gamma\left(X, f_{*} \mathcal{O}_{Y}\right)\right)$ is equal to the element $\Psi(Y / X)$ of $K_{0}(k[G])$ which is defined by Chinburg in ([Ch], Definition 3.3 and Proposition 4.1(a)). The study of $\Psi(Y / X)$ in loc. cit. may therefore be interpreted as being the study of the image of the element $\left(f_{*} \mathcal{O}_{Y}\right)$ of $K_{0}^{l f}\left(\mathcal{O}_{X}[G]\right)$ under the homomorphism $\chi R \Gamma_{\mathcal{O}_{X}[G], X}$ of Proposition 4.4(b). From this point of view, the subgroup $D^{s f}\left(\mathcal{O}_{X}[G]\right)$ provides some measure of the fact that $\left(f_{*} \mathcal{O}_{Y}\right)$ is a finer invariant than $\Psi(Y / X)$.

The connection between the groups $D^{s s}(\mathcal{A})$ and $D^{s f}(\mathcal{A})$ is described by the following result.

PROPOSITION 4.6. Suppose that $\mathcal{A}$ is isotrivial and $\mathcal{A}_{s s}(X)$ has the Wedderburn decomposition (1.1), and set

$$
\mathcal{A}_{s s}^{\prime}:=\prod_{i \in I}\left(f_{m(i)}\right)_{*} \mathcal{O}_{X_{m(i)}}
$$

Let $\hat{\sigma}_{\mathcal{A}}$ denote the composite map

$$
K_{0}^{l f}(\mathcal{A}) \rightarrow K_{0}^{l f}\left(\mathcal{A}_{s s}\right) \rightarrow K_{0}^{l f}\left(\mathcal{A}_{s s}^{\prime}\right),
$$

where the first morphism is $\sigma_{\mathcal{A}}$ and the second morphism is induced by (1.2) and the morphisms $G_{\mathcal{A}_{s, i}^{\prime}, \text { n(i) }}^{0}$ of Proposition 3.8. If either $X$ is a curve or $\mathcal{A}_{s s}$ is of Swan-type, then there is an exact sequence of groups

$$
0 \rightarrow D^{s s}(\mathcal{A}) \rightarrow D^{s f}(\mathcal{A}) \rightarrow \bigoplus_{i \in I} D^{s f}\left(\mathcal{O}_{X_{m(i)}}\right) \rightarrow \operatorname{Coker}\left(\hat{\sigma}_{\mathcal{A}}\right) .
$$

Proof. The key observation that we use is provided by the following lemma.

LEMMA 4.7. If $\mathcal{A}$ is isotrivial, then for each $U \in \mathcal{U}(X)$ the following diagram commutes:

$$
\begin{aligned}
& \begin{array}{cc}
K_{0}^{l f}(\mathcal{A}) \stackrel{\chi R \Gamma_{\mathcal{A}, U}}{\longrightarrow} & K_{0}(\mathcal{A}(U)) \\
\downarrow \mathcal{A}_{s s} \otimes_{\mathcal{A}^{-}} & \downarrow \mathcal{A}_{s s}(U) \otimes_{\mathcal{A}(U)^{-}}
\end{array} \\
& K_{0}^{l f}\left(\mathcal{A}_{s s}\right) \stackrel{\chi R \Gamma_{\mathcal{A}_{s s}, U}}{\longrightarrow} K_{0}\left(\mathcal{A}_{s s}(U)\right) .
\end{aligned}
$$

Proof. We set $\Lambda:=\mathcal{A}(X), \Lambda_{s s}:=\mathcal{A}(X) / \operatorname{Jac}(\mathcal{A}(X))$ and $\mathcal{V}_{s s}:=\mathcal{A}_{s s} \otimes_{\mathcal{A}} \mathcal{V}$ for each $\mathcal{A}$-bundle $\mathcal{V}$. 
If $U \in \mathcal{U}^{a f f}(X)$, then $\mathcal{V}_{s s}(U)=\Lambda_{s s} \otimes_{\Lambda} \mathcal{V}(U)$ for each $\mathcal{A}$-bundle $\mathcal{V}$ and this clearly ensures that the diagram commutes. To show that the diagram commutes when $U=X$, it suffices to show that there is an isomorphism

$$
\Lambda_{s s} \otimes_{\Lambda}^{L} R \Gamma(X, \mathcal{V}) \cong R \Gamma\left(X, \mathcal{V}_{s s}\right)
$$

in $\mathfrak{D}^{b}\left(\Lambda_{s s}\right)$. In order to prove that such an isomorphism holds, we describe $R \Gamma(X$, $\mathcal{V})$ and $R \Gamma\left(X, \mathcal{V}_{s s}\right)$ in terms of Čech cohomology, just as in the proof of Proposition 4.4 .

We choose a finite affine open covering $\mathcal{U}=\left\{U_{i}\right\}$ of $X$, and a bounded complex of finitely generated projective $\Lambda$-modules $P^{\bullet}$ which is isomorphic in $\mathfrak{D}^{b}(\Lambda)$ to $C^{\bullet}(\mathcal{U}, \mathcal{V})$. Since both $P^{\bullet}$ and $C^{\bullet}(\mathcal{U}, \mathcal{V})$ are bounded complexes of projective $\Lambda$-modules they are in fact $\Lambda$-equivariantly homotopy equivalent. This implies that the complexes $\Lambda_{s s} \otimes_{\Lambda} P^{\bullet}$ and $\Lambda_{s s} \otimes_{\Lambda} C^{\bullet}(\mathcal{U}, \mathcal{V})$ are isomorphic in $\mathfrak{D}^{b}\left(\Lambda_{s s}\right)$. To prove that there is an isomorphism as in (4.7), it is therefore enough to show that the complexes $\Lambda_{s s} \otimes_{\Lambda} C^{\bullet}(\mathcal{U}, \mathcal{V})$ and $C^{\bullet}\left(\mathcal{U}, \mathcal{V}_{s s}\right)$ are isomorphic in $\mathfrak{D}^{b}\left(\Lambda_{s s}\right)$. This is in turn an easy consequence of the fact that each intersection $U=$ $U_{i_{0}} \cap U_{i_{1}} \cap \ldots \cap U_{i_{s}}$ is an affine open subset of $X$ (since $X$ is separated), and therefore $\Lambda_{s s} \otimes_{\Lambda} \mathcal{V}(U)=\mathcal{V}_{s s}(U)$.

Taken together, the commutative diagrams of Lemma 4.7 give rise to the following commutative diagram, in which both rows and the central column are exact:

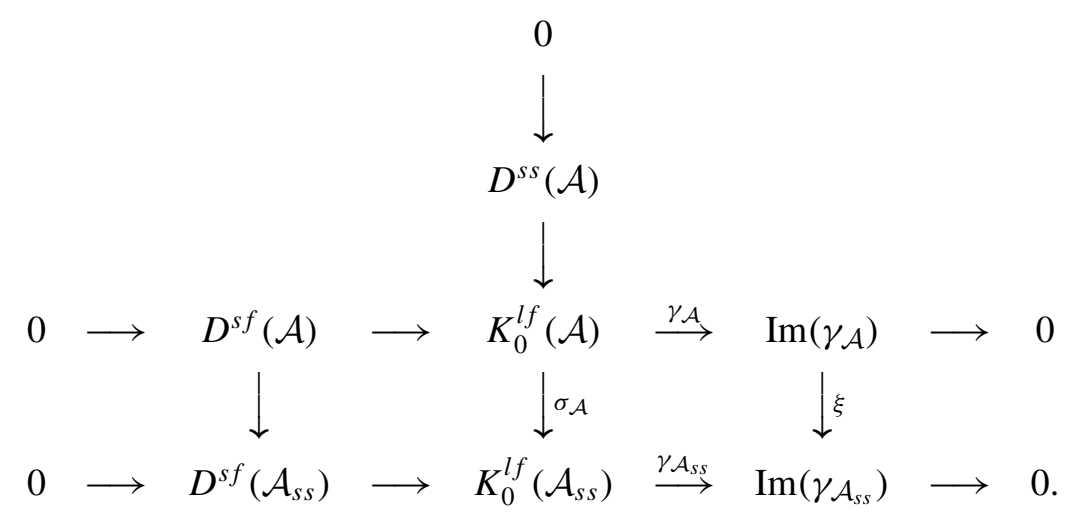

Here $\xi$ denotes the morphism induced by the functor $\prod_{U \in \mathcal{U}(X)}\left(\mathcal{A}_{s s}(U) \otimes_{\mathcal{A}(U)}-\right)$. Grothendieck's Devissage theorem (cf. [B], Chap. VIII, 4.2) implies that $\xi$ is injective, and so by applying the Snake lemma to this commutative diagram, one obtains an exact sequence

$$
0 \rightarrow D^{s s}(\mathcal{A}) \rightarrow D^{s f}(\mathcal{A}) \rightarrow D^{s f}\left(\mathcal{A}_{s s}\right) \rightarrow \operatorname{Coker}\left(\sigma_{\mathcal{A}}\right) \rightarrow \operatorname{Coker}(\xi) \rightarrow 0 .
$$

With respect to the decomposition of $\mathcal{A}_{s s}$ given by (1.2), we set

$$
\mathcal{A}_{s s, i}^{\prime}:=\left(f_{m(i)}\right)_{*} \mathcal{O}_{X_{m(i)}}
$$


and

$$
\mathcal{A}_{s s, i}:=M_{n(i)}\left(\mathcal{A}_{s s, i}^{\prime}\right)
$$

for each $i \in I$. We let $\mathcal{P}_{i}$ denote the direct sum of $n(i)$ copies of $\mathcal{A}_{s s, i}^{\prime}$ for each $i \in I$. Recall from Proposition 3.8(a) that the functors

$$
G_{\mathcal{P}_{i}}: \mathfrak{B}^{l f}\left(\mathcal{A}_{s s, i}\right) \rightarrow \mathfrak{B}^{l f}\left(\mathcal{A}_{s s, i}^{\prime}\right)
$$

induce injective morphisms

$$
G_{i}^{0}:=G_{\mathcal{A}_{s, i}^{\prime}, n(i)}^{0}: K_{0}^{l f}\left(\mathcal{A}_{s s, i}\right) \rightarrow K_{0}^{l f}\left(\mathcal{A}_{s s, i}^{\prime}\right) .
$$

Let $U$ be any affine open subset of $X$, and suppose that $\mathcal{V} \in \mathfrak{B}^{l f}\left(\mathcal{A}_{s s, i}\right)$. Since

$$
\mathcal{A}_{s s, i} \cong \mathcal{A}_{s s, i}^{\prime} \otimes_{k} M_{n(i)}(k),
$$

one has a natural isomorphism of $k$-modules

$$
\mathcal{P}_{i}(U) \otimes_{\mathcal{A}_{s s, i}(U)} \mathcal{V}(U) \cong k^{n(i)} \otimes_{M_{n(i)}(k)} \mathcal{V}(U) .
$$

In fact, since the functor $k^{n(i)} \otimes_{M_{n(i)}(k)}$ - is exact, this implies that

$$
G_{\mathcal{P}_{i}}(\mathcal{V})(U) \cong k^{n(i)} \otimes_{M_{n(i)}(k)} \mathcal{V}(U)
$$

for each open subset $U$ of $X$. By using this description one can show that the following diagram commutes for each $U \in \mathcal{U}(X)$ and $i \in I$ :

$$
\begin{array}{ccc}
K_{0}^{l f}\left(\mathcal{A}_{s s, i}\right) & \stackrel{\chi R \Gamma_{\mathcal{A}_{s s, i}, U}}{\longrightarrow} & K_{0}\left(\mathcal{A}_{s s, i}(U)\right) \\
\downarrow G_{i}^{0} & & \downarrow \mathcal{P}_{i}(U) \otimes_{\mathcal{A}_{s s, i}(U)-} \\
K_{0}^{l f}\left(\mathcal{A}_{s s, i}^{\prime}\right) & \stackrel{\chi R \Gamma_{\mathcal{A}_{s s, i}^{\prime}, U}}{\longrightarrow} & K_{0}\left(\mathcal{A}_{s s, i}^{\prime}(U)\right) .
\end{array}
$$

Indeed, whilst this follows immediately from (4.9) if $U$ is affine (so that both $\chi R \Gamma_{\mathcal{A}_{s s, i}, U}(-)$ and $\chi R \Gamma_{\mathcal{A}_{s, i}^{\prime}, U}(-)$ are obtained by taking sections over $U$ ), in the case $U=X$ it follows from (4.9) and a computation of $\chi R \Gamma_{\mathcal{A}_{s s, i}, X}$ and $\chi R \Gamma_{\mathcal{A}_{s, i}^{\prime}, X}$ using Čech cohomology just as in the proof of Lemma 4.7.

The left-hand (respectively right-hand) vertical map in the last displayed commuting diagram is injective (respectively bijective). By combining these diagrams for each $U \in \mathcal{U}(X)$ and $i \in I$ and then using the Snake Lemma, one concludes that there is an exact sequence

$$
0 \rightarrow D^{s f}\left(\mathcal{A}_{s s}\right) \rightarrow D^{s f}\left(\mathcal{A}_{s s}^{\prime}\right) \rightarrow \operatorname{Coker}\left(K_{0}^{l f}\left(\mathcal{A}_{s s}\right) \rightarrow K_{0}^{l f}\left(\mathcal{A}_{s s}^{\prime}\right)\right) .
$$

By combining this sequence with (4.8) one derives an exact sequence

$$
0 \rightarrow D^{s s}(\mathcal{A}) \rightarrow D^{s f}(\mathcal{A}) \rightarrow D^{s f}\left(\mathcal{A}_{s s}^{\prime}\right) \rightarrow \operatorname{Coker}\left(\hat{\sigma}_{\mathcal{A}}\right) .
$$


To obtain the exact sequence of Proposition 4.6 it is now sufficient to observe that $D^{s f}\left(\mathcal{A}_{s s, i}^{\prime}\right) \cong D^{s f}\left(\mathcal{O}_{X_{m(i)}}\right)$ for each $i \in I$. These isomorphisms follow from the category equivalence of Proposition 3.9 and the fact that $\operatorname{Spec}\left(\mathcal{A}_{s s, i}^{\prime}\right) \cong X_{m(i)}$.

At the end of the next section we will use Proposition 4.6 to prove the following result.

THEOREM 4.8. Let $X$ be a curve of genus $g$. Let $\mathcal{A}$ be an isotrivial sheaf of orders on $X$, and assume that $\mathcal{A}_{s s}(X)$ has the Wedderburn decomposition (1.1). Then $D^{s f}(\mathcal{A})$ is finite and

$$
\# D^{s f}(\mathcal{A}) \leqslant(\# k)^{g \cdot \operatorname{dim}_{k}(\mathcal{R}(X))} \prod_{i \in I} \# \operatorname{Pic}^{0}(X)\left(k_{m(i)}\right) .
$$

In order to state our next result, we first recall that the Grothendieck $\gamma$-filtration on each of the groups $K_{0}\left(X_{m(i)}\right)$ has length at most $\operatorname{dim}(X)+1$. Hence the results of Propositions 3.8 and 3.9 imply that this induces a decreasing filtration $F_{\gamma}^{i} K_{0}^{l f}\left(\mathcal{A}_{s s}\right)$ on $K_{0}^{l f}\left(\mathcal{A}_{s s}\right)$ of length at most $\operatorname{dim}(X)+1$. By using the next result, one can obtain an interpretation of the first graded piece of this filtration in terms of Galois-equivariant functions with values in $\operatorname{Pic}\left(X^{c}\right)$. This will be very useful in later sections.

Set $\Omega_{k}:=\operatorname{Gal}\left(k^{c} / k\right)$.

LEMMA 4.9. Let $k^{\prime}$ be a finite extension of $k$ in $k^{c}$. Set $X^{\prime}:=X \times_{k} k^{\prime}$ and $X^{c}:=$ $X \times_{k} k^{c}$. Then there are canonical isomorphisms

$$
G r_{\gamma}^{1} K_{0}\left(X^{\prime}\right) \cong \operatorname{Pic}\left(X^{\prime}\right) \cong \operatorname{Hom}_{\Omega_{k}}\left(\mathbb{Z}\left[\operatorname{Gal}\left(k^{\prime} / k\right)\right], \operatorname{Pic}\left(X^{c}\right)\right) .
$$

Proof. The existence of the isomorphism $G r_{\gamma}^{1} K_{0}\left(X^{\prime}\right) \cong \operatorname{Pic}\left(X^{\prime}\right)$ is a standard result, and a proof is given on p. 126 of $[\mathrm{Fu}, \mathrm{L}]$. On the other hand, $\operatorname{Pic}\left(X^{\prime}\right) \cong$ $H^{1}\left(X_{e t}^{\prime}, \mathbb{G}_{m}\right)$ and $H^{1}\left(\Omega_{k^{\prime}}, k^{c *}\right)=\{0\}$ (by Hilbert's Theorem 90); since also $H^{2}\left(\Omega_{k^{\prime}}, k^{c *}\right)=\{0\}$ (because there are no non-commutative, finite division rings), the Hochschild-Serre spectral sequence of ([Mi], Chap. III, Th. 2.20) induces an isomorphism $\operatorname{Pic}\left(X^{\prime}\right) \cong H^{0}\left(\Omega_{k^{\prime}}, \operatorname{Pic}\left(X^{c}\right)\right)$. The claimed isomorphism

$$
\operatorname{Pic}\left(X^{\prime}\right) \cong \operatorname{Hom}_{\Omega_{k}}\left(\mathbb{Z}\left[\operatorname{Gal}\left(k^{\prime} / k\right)\right], \operatorname{Pic}\left(X^{c}\right)\right),
$$

thus follows from the isomorphism of functors

$$
H^{0}\left(\Omega_{k^{\prime}},-\right) \cong \operatorname{Map}_{\Omega_{k}}\left(\operatorname{Gal}\left(k^{\prime} / k\right),-\right) \cong \operatorname{Hom}_{\Omega_{k}}\left(\mathbb{Z}\left[\operatorname{Gal}\left(k^{\prime} / k\right)\right],-\right) .
$$

Remark 4.10. For each integer $i \geqslant 0$ we define $F^{i} K_{0}^{l f}(\mathcal{A})$ to be equal to the inverse image of $F_{\gamma}^{i} K_{0}^{l f}\left(\mathcal{A}_{s s}\right)$ under $\sigma_{\mathcal{A}}$ if $i \leqslant \operatorname{dim}(X)+1$ and to be equal to $D_{i-\operatorname{dim}(X)}^{s s}(\mathcal{A})$ if $i>\operatorname{dim}(X)+1$. Let $e$ be the smallest non-negative integer such that $\mathcal{R}(\mathcal{A})^{e}$ is zero (see Lemma 1.4(c)). Then the subgroups $\left\{F^{i} K_{0}^{l f}(\mathcal{A})\right\}_{i} \geqslant 0$ give an exhaustive, separated, decreasing filtration of $K_{0}^{l f}(\mathcal{A})$ which is of length at 
most $\operatorname{dim}(X)+e$. Note that Lemma 1.4(c) implies $e$ is less than or equal to the $K$-dimension of the generic stalk of $\mathcal{A}$.

\section{IDELIC DESCRIPTIONS}

Throughout this section, $X$ is a curve (i.e. an irreducible non-singular projective variety of dimension one over $k$, with $k=\mathcal{O}(X)$ ), and $\mathcal{A}$ is a sheaf of orders on $X$. We write $A$ for the generic stalk of $\mathcal{A}$.

DEFINITION 5.1. (a) Define the idele group $J\left(K_{1}(A)\right)$ of $K_{1}(A)$ by

$$
\begin{aligned}
& J\left(K_{1}(A)\right):= \\
& \left\{\left(\alpha_{x}\right) \in \prod_{x \in X_{0}} K_{1}\left(A_{\hat{x}}\right) \mid \alpha_{x} \in \operatorname{Im}\left(\iota_{x}\right) \text { for all but finitely many } x \in X_{0}\right\},
\end{aligned}
$$

where, for each $x \in X_{0}$,

$$
\iota_{x}: K_{1}\left(\mathcal{A}_{\hat{x}}\right) \rightarrow K_{1}\left(A_{\hat{x}}\right)
$$

denotes the morphism induced by the natural scalar extension functor.

Note that given any $\alpha \in G L_{n}(A)$, there exists an affine open subset $U$ of $X$ such that $\alpha \in G L_{n}(\mathcal{A}(U))$. Hence there exists a diagonal morphism

$$
\Delta_{1}: K_{1}(A) \rightarrow J\left(K_{1}(A)\right) .
$$

(b) Define the idele group $J(A)$ of $A$ by

$$
J(A):=\left\{\left(v_{x}\right) \in \prod_{x \in X_{0}} A_{\hat{x}}^{*} \mid v_{x} \in \mathcal{A}_{\hat{x}}^{*} \text { for all but finitely many } x \in X_{0}\right\},
$$

and write

$$
\Delta_{2}: A^{*} \rightarrow J(A)
$$

for the natural diagonal map.

In this section we shall use the exact sequences (3.2) and (3.3) to give a natural description of $\mathrm{Cl}^{l f}(\mathcal{A})$ in terms of the idele group $J\left(K_{1}(A)\right)$, and we shall then derive some useful consequences of this description.

THEOREM 5.2. Suppose that $X$ is a curve, and that $\mathcal{A}$ is a sheaf of orders on $X$. Then there is a natural isomorphism of groups

$$
\mathrm{Cl}^{l f}(\mathcal{A}) \simeq \frac{J\left(K_{1}(A)\right)}{\operatorname{Im}\left(\Delta_{1}\right) \cdot \prod_{x \in X_{0}} \operatorname{Im}\left(\iota_{x}\right)} .
$$

Proof. If $\mathcal{F}_{x}$ is any $\mathcal{A}_{x}$-module, we let $i_{x}\left(\mathcal{F}_{x}\right)$ denote the corresponding skyscraper sheaf on $X$. 
We require the following lemma. (We remind the reader that the categories $\mathfrak{H}(\mathcal{A})$ and $H\left(\mathcal{A}_{\hat{x}}\right)$ were described in Definition 3.10.)

LEMMA 5.3. For any $\mathcal{A}$-module $\mathcal{T}$ which is a coherent, torsion $\mathcal{O}$-module, there is a natural isomorphism $\mathcal{T} \stackrel{\sim}{\rightarrow} \oplus_{x \in X_{0}} i_{x}\left(\mathcal{T}_{x}\right)$. Furthermore, $\mathcal{T}$ belongs to $\mathfrak{H}(\mathcal{A})$ if and only if $\mathcal{T}_{\hat{x}}$ belongs to $H\left(\mathcal{A}_{\hat{x}}\right)$ for each $x \in X_{0}$.

Proof. We first observe that the natural morphism $\mathcal{T} \rightarrow \prod_{x \in X_{0}} i_{x}\left(\mathcal{T}_{x}\right)$ induces an isomorphism between $\mathcal{T}$ and $\oplus_{x \in X_{0}} i_{x}\left(\mathcal{T}_{x}\right)$ since the support of $\mathcal{T}$ is finite (cf. $[\mathrm{H}]$, Chap. II, Ex. 5.6(c)).

Now suppose that $\mathcal{T} \in \mathfrak{H}(\mathcal{A})$ and let

$$
0 \rightarrow \mathcal{P}^{-1} \rightarrow \mathcal{P}^{0} \rightarrow \mathcal{T} \rightarrow 0
$$

be a short exact sequence with $\mathcal{P}^{-1}$ and $\mathcal{P}^{0}$ both $\mathcal{A}$-bundles on $X$. We deduce that $\mathcal{T}_{\hat{x}} \in H\left(\mathcal{A}_{\hat{x}}\right)$ for each $x \in X_{0}$ by taking completions of stalks of this sequence.

To complete the proof of the lemma, we must show that if $\mathcal{T}_{\hat{x}} \in H\left(\mathcal{A}_{\hat{x}}\right)$, then $i_{x}\left(\mathcal{T}_{x}\right) \in \mathfrak{H}(\mathcal{A})$. By Lemma 2.5 there is an $\mathcal{A}$-bundle $\mathcal{P}^{0}$ on $X$ and an epimorphism $\pi: \mathcal{P}^{0} \rightarrow i_{x}\left(\mathcal{T}_{x}\right)$. Setting $\mathcal{P}^{-1}:=\operatorname{Ker}(\pi)$, it suffices to show that $\mathcal{P}_{y}^{-1}$ is a free $\mathcal{A}_{y}$-module for each $y \in X$. If $y \neq x$, then $\mathcal{P}_{y}^{-1}=\mathcal{P}_{y}^{0}$, and so $\mathcal{P}_{y}^{-1}$ is free. If $y=x$, then we argue as follows. Since $\mathcal{T}_{\hat{x}} \in H\left(\mathcal{A}_{\hat{x}}\right)$, there is an exact sequence

$$
0 \rightarrow \mathcal{F}_{\hat{x}}^{-1} \rightarrow \mathcal{F}_{\hat{x}}^{0} \rightarrow \mathcal{T}_{\hat{x}} \rightarrow 0
$$

in which $\mathcal{F}_{\hat{x}}^{-1}$ and $\mathcal{F}_{\hat{x}}^{0}$ are finitely generated free $\mathcal{A}_{\hat{x}}$-modules. Since we also have an exact sequence

$$
0 \rightarrow \mathcal{P}_{\hat{x}}^{-1} \rightarrow \mathcal{P}_{\hat{x}}^{0} \rightarrow \mathcal{T}_{\hat{x}} \rightarrow 0,
$$

it follows from Schanuel's Lemma that the $\mathcal{A}_{\hat{x}}$-modules $\mathcal{F}_{\hat{x}}^{-1} \oplus \mathcal{P}_{\hat{x}}^{0}$ and $\mathcal{F}_{\hat{x}}^{0} \oplus \mathcal{P}_{\hat{x}}^{-1}$ are isomorphic. As $\mathcal{P}_{\hat{x}}^{0}$ is a free $\mathcal{A}_{\hat{x}}$-module, the Krull-Schmidt-Azumaya theorem (cf. [C, R1], Th. 6.12(ii)) therefore implies that $\mathcal{P}_{\hat{x}}^{-1}$ is $\mathcal{A}_{\hat{x}}$-free, whence it follows that $\mathcal{P}_{x}^{-1}$ is a free $\mathcal{A}_{x}$-module (cf. ibid., Prop. 30.17).

This lemma implies that the map

$$
\phi: K_{0}(\mathfrak{H}(\mathcal{A})) \longrightarrow \coprod_{x \in X_{0}} K_{0}\left(H\left(\mathcal{A}_{\hat{x}}\right)\right),
$$

which is induced by sending $(\mathcal{T})$ to $\oplus_{x \in X_{0}}\left(\mathcal{T}_{\hat{x}}\right)$ is an isomorphism. In the following diagram, $\iota$ denotes the morphism $K_{1}^{l f}(\mathcal{A}) \rightarrow K_{1}(A)$ induced by taking generic stalks, the upper row is a consequence of (3.2) and is exact, the lower row is a consequence of (3.3) and is exact, and the map $\psi$ is induced by the diagonal morphisms $\Delta_{1}: K_{1}(A) \longrightarrow \prod_{x \in X_{0}} K_{1}\left(A_{\hat{x}}\right)$ and $K_{1}^{l f}(\mathcal{A}) \longrightarrow \prod_{x \in X_{0}} K_{1}\left(\mathcal{A}_{\hat{x}}\right)$ :

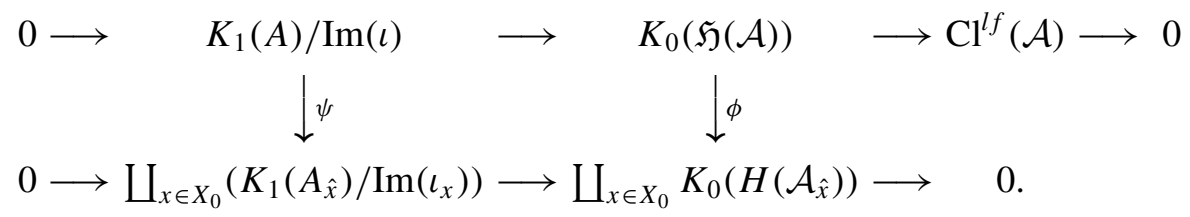


This diagram is commutative and so, by applying the Snake lemma, we obtain an exact sequence

$$
0 \rightarrow K_{1}(A) / \operatorname{Im}(\iota) \stackrel{\psi}{\rightarrow} \coprod_{x \in X_{0}}\left(K_{1}\left(A_{\hat{x}}\right) / \operatorname{Im}\left(\iota_{x}\right)\right) \rightarrow \mathrm{Cl}^{l f}(\mathcal{A}) \rightarrow 0 .
$$

This implies Theorem 5.2.

Before stating the next result we recall that there is a natural bijection between the pointed set $H^{1}\left(X, \mathcal{A}^{*}\right)$ and the set of isomorphism classes of rank one $\mathcal{A}$ bundles (see Lemma 2.4). By regarding $\mathrm{Cl}^{l f}(\mathcal{A})$ as a quotient of $K_{0}^{l f}(\mathcal{A})$ it follows that there is a natural map

$$
\lambda_{\mathcal{A}}: H^{1}\left(X, \mathcal{A}^{*}\right) \rightarrow \mathrm{Cl}^{l f}(\mathcal{A})
$$

of pointed sets.

COROLLARY 5.4. If $X$ is a curve and $\mathcal{A}$ is a sheaf of orders on $X$, then the map $\lambda_{\mathcal{A}}$ is surjective.

Proof. Since $X$ is irreducible, each rank one $\mathcal{A}$-bundle is isomorphic to an $\mathcal{A}$ subsheaf of the constant sheaf $A$ (cf. [H], Chap. II, proof of Prop. 6.15). Such a subsheaf $\mathcal{V}$ gives an idele $\left\{v_{x}\right\}_{x \in X_{0}} \in J(A)$ by the condition $\mathcal{V}_{\hat{x}}=\mathcal{A}_{\hat{x}} v_{x}$, and this idele is well defined only up to left multiplication by an element of $\prod_{x \in X_{0}} \mathcal{A}_{\hat{x}}^{*}$. As two such sheaves $\mathcal{V}_{1}$ and $\mathcal{V}_{2}$ are isomorphic if and only if $\mathcal{V}_{1}=\mathcal{V}_{2} a$ for some $a \in$ $A^{*}$, it follows that there is a canonical injective map from the set of isomorphism classes of rank one $\mathcal{A}$-bundles to the set of double cosets $\prod_{x \in X_{0}} \mathcal{A}_{\hat{x}}^{*} \backslash J(A) / \operatorname{Im}\left(\Delta_{2}\right)$.

We claim that this map is bijective. To show this, we observe that corresponding to any idele $\left(a_{x}\right) \in J(A)$, there exists an $\mathcal{A}$-bundle defined in the following way. For any affine open subset $U$ of $X$, there exists a unique $\mathcal{O}(U)$-lattice $\mathcal{L}(U)$ in $A$ such that $\mathcal{L}(U)_{\hat{x}}=\mathcal{A}_{\hat{x}} a_{x}$ for each $x \in U \cap X_{0}$ (cf. [R], Th. 4.22). Since $\mathcal{O}_{x}$ is a discrete valuation ring, it follows that $\mathcal{L}(U)_{x}$ is a free $\mathcal{A}(U)_{x}$-module (see [C, R1], Prop. 30.17). The $\mathcal{A}(U)$-modules $\mathcal{L}(U)$ glue together to give an $\mathcal{A}$-bundle $\mathcal{L}$ on $X$ whose image in $\prod_{x \in X_{0}} \mathcal{A}_{\hat{x}}^{*} \backslash J(A) / \operatorname{Im}\left(\Delta_{2}\right)$ under the above map is represented by $\left(a_{x}\right) \in J(A)$.

For each $x \in X_{0}$, the rings $A_{\hat{x}}$ and $\mathcal{A}_{\hat{x}}$ are semi-local and hence the natural maps $A_{\hat{x}}^{*} \rightarrow K_{1}\left(A_{\hat{x}}\right)$ and $\mathcal{A}_{\hat{x}}^{*} \rightarrow K_{1}\left(\mathcal{A}_{\hat{x}}\right)$ are surjective (cf. [C, R2],(40.31)). These maps combine to give a surjection $J(A) \rightarrow J\left(K_{1}(A)\right)$, and the stated result now follows as a consequence of Theorem 5.2.

COROLLARY 5.5. Let $X$ be a curve, and suppose that $\mathcal{A}$ is an isotrivial sheaf of orders on X. Let

$$
\tau: H^{1}\left(X, \mathcal{A}^{*}\right) \rightarrow H^{1}\left(X, \mathcal{A}_{s s}^{*}\right)
$$

be the homomorphism (of pointed sets) induced by the quotient map $\mathcal{A}^{*} \rightarrow \mathcal{A}_{s s}^{*}$. Then $\tau\left(\operatorname{Ker}\left(\lambda_{\mathcal{A}}\right)\right)=\operatorname{Ker}\left(\lambda_{\mathcal{A}_{s s}}\right)$. 
Proof. Set $\mathcal{B}:=\mathcal{A}_{s s}$ and let $B$ denote its generic stalk. Note that, since $\mathcal{A}$ is isotrivial, $B$ is equal to the quotient of $A$ by the generic stalk of $\mathcal{R}(\mathcal{A})$. For each $x \in X_{0}$, let

$$
\varepsilon_{x}: \mathcal{A}_{\hat{x}}^{*} \rightarrow K_{1}\left(\mathcal{A}_{\hat{x}}\right)
$$

and

$$
\varphi_{x}: A_{\hat{x}}^{*} \rightarrow K_{1}\left(A_{\hat{x}}\right)
$$

denote the natural surjections which result from the fact that $\mathcal{A}_{\hat{x}}$ and $A_{\hat{x}}$ are semilocal rings (see the last paragraph of the proof of Corollary 5.4). Write

$$
\begin{aligned}
\theta_{1}: & \prod_{x \in X_{0}} \mathcal{A}_{\hat{x}}^{*} \rightarrow \prod_{x \in X_{0}} \operatorname{Im}\left(\iota_{x}\right), \\
\theta_{2}: & J(A) \rightarrow J\left(K_{1}(A)\right), \\
\theta_{3}: & \prod_{x \in X_{0}} \mathcal{A}_{\hat{x}}^{*} \backslash J(A) \rightarrow \prod_{x \in X_{0}} \operatorname{Im}\left(\iota_{x}\right) \backslash J\left(K_{1}(A)\right),
\end{aligned}
$$

and

$$
\theta_{4}: \operatorname{Im}\left(\Delta_{2}\right) \rightarrow \operatorname{Im}\left(\Delta_{1}\right)
$$

for the obvious surjections induced by the maps $\varphi_{x}$ and $\varepsilon_{x}$ for each $x \in X_{0}$. Let

$$
\theta_{5}: \prod_{x \in X_{0}} \mathcal{A}_{\hat{x}}^{*} \backslash J(A) / \operatorname{Im}\left(\Delta_{2}\right) \rightarrow \mathrm{Cl}^{l f}(\mathcal{A})
$$

denote the surjection induced by $\theta_{3}, \theta_{4}$, and the isomorphism of Theorem 5.2.

Since $\mathcal{A}$ is isotrivial, Lemma 1.8(a) implies that $\mathcal{B}$ is an isotrivial sheaf of orders. Let $l_{x}^{s s}, \varepsilon_{x}^{s s}, \varphi_{x}^{s s}$, and $\theta_{i}^{s s}(1 \leqslant i \leqslant 5)$ denote the maps analogous to those above which are obtained by replacing $\mathcal{A}$ and $A$ by $\mathcal{B}$ and $B$, respectively. For ease of notation, we set

$$
\mathfrak{J}(A):=\prod_{x \in X_{0}} \mathcal{A}_{\hat{x}}^{*} \backslash J(A) / \operatorname{Im}\left(\Delta_{2}\right) .
$$

We shall require the following lemma.

LEMMA 5.6. There are the following natural isomorphisms of pointed sets:

(a) $\operatorname{Ker}\left(\lambda_{\mathcal{A}}\right) \simeq \operatorname{Ker}\left(\theta_{1}\right) \backslash \operatorname{Ker}\left(\theta_{2}\right) / \operatorname{Ker}\left(\theta_{4}\right)$;

(b) $\operatorname{Ker}\left(\lambda_{\mathcal{B}}\right) \simeq \operatorname{Ker}\left(\theta_{1}^{s s}\right) \backslash \operatorname{Ker}\left(\theta_{2}^{s s}\right) / \operatorname{Ker}\left(\theta_{4}^{s s}\right)$.

Proof. (a) Recall from the proof of Corollary 5.4 that there is a natural bijection $H^{1}\left(X, \mathcal{A}^{*}\right) \simeq \mathfrak{J}(A)$. Hence we have the following commutative diagram of pointed 
sets, in which both rows are exact, and the second and third vertical arrows are isomorphisms:

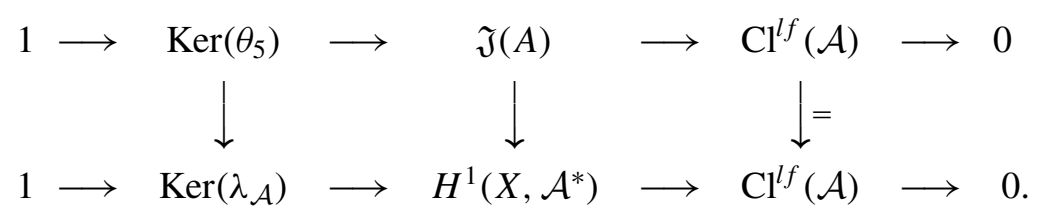

This implies that there is an isomorphism

$$
\operatorname{Ker}\left(\lambda_{\mathcal{A}}\right) \simeq \operatorname{Ker}\left(\theta_{5}\right),
$$

of pointed sets. We shall now show that there is an isomorphism

$$
\operatorname{Ker}\left(\theta_{5}\right) \simeq \operatorname{Ker}\left(\theta_{1}\right) \backslash \operatorname{Ker}\left(\theta_{2}\right) / \operatorname{Ker}\left(\theta_{4}\right),
$$

of pointed sets. To do this, we first observe that the following diagram of pointed sets (in which the rows are exact and the vertical arrows are surjective) is commutative:

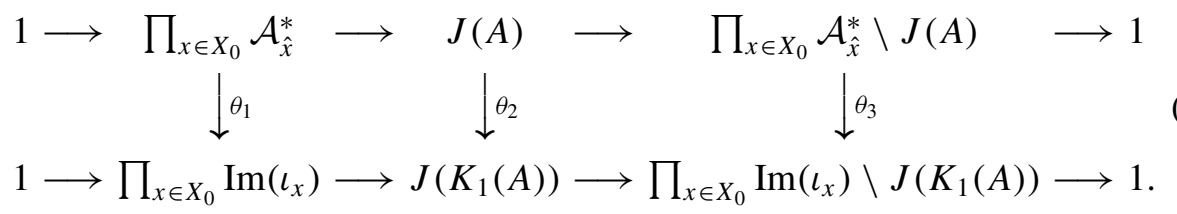

Applying the Snake lemma to (5.3) gives the following exact sequence of pointed sets:

$$
1 \rightarrow \operatorname{Ker}\left(\theta_{1}\right) \rightarrow \operatorname{Ker}\left(\theta_{2}\right) \rightarrow \operatorname{Ker}\left(\theta_{3}\right) \rightarrow 1,
$$

and so it follows that

$$
\operatorname{Ker}\left(\theta_{3}\right) \simeq \operatorname{Ker}\left(\theta_{1}\right) \backslash \operatorname{Ker}\left(\theta_{2}\right) .
$$

Next we consider the following commutative diagram of pointed sets (in which the rows are exact, the vertical arrows are surjective and the third arrow on the bottom row is the map afforded by Theorem 5.2):

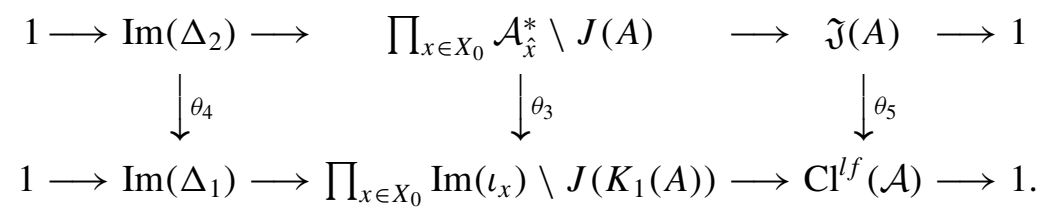

Applying the Snake lemma to (5.5) yields the exact sequence

$$
1 \rightarrow \operatorname{Ker}\left(\theta_{4}\right) \rightarrow \operatorname{Ker}\left(\theta_{3}\right) \rightarrow \operatorname{Ker}\left(\theta_{5}\right) \rightarrow 1 .
$$


Hence (5.6) and (5.4) imply that

$$
\operatorname{Ker}\left(\theta_{5}\right) \simeq \operatorname{Ker}\left(\theta_{3}\right) / \operatorname{Ker}\left(\theta_{4}\right) \simeq \operatorname{Ker}\left(\theta_{1}\right) \backslash \operatorname{Ker}\left(\theta_{2}\right) / \operatorname{Ker}\left(\theta_{4}\right),
$$

and so it follows from (5.1) that

$$
\operatorname{Ker}\left(\lambda_{\mathcal{A}}\right) \simeq \operatorname{Ker}\left(\theta_{1}\right) \backslash \operatorname{Ker}\left(\theta_{2}\right) / \operatorname{Ker}\left(\theta_{4}\right)
$$

as claimed.

(b) This follows by an argument very similar to that given in part (a).

Now write

$$
\begin{aligned}
& q: J(A) \rightarrow J(B), \\
& Q_{x}: \operatorname{Ker}\left(\varphi_{x}\right) \rightarrow \operatorname{Ker}\left(\varphi_{x}^{s s}\right),
\end{aligned}
$$

and

$$
Q_{x}^{\prime}: \operatorname{Ker}\left(\varepsilon_{x}\right) \rightarrow \operatorname{Ker}\left(\varphi_{x}^{s s} \mid \mathcal{B}_{\hat{x}}^{*}\right)
$$

for the homomorphisms induced by the quotient map $\mathcal{A} \rightarrow \mathcal{B}$. Lemma $5.6 \mathrm{im}$ plies that the assertion that $\tau\left(\operatorname{Ker}\left(\lambda_{\mathcal{A}}\right)\right)=\operatorname{Ker}\left(\lambda_{\mathcal{B}}\right)$ will follow if we show that $q\left(\operatorname{Ker}\left(\theta_{2}\right)\right)=\operatorname{Ker}\left(\theta_{2}^{s s}\right)$. This will in turn follow if we show that $Q_{x}$ is surjective for all $x \in X_{0}$ and that $Q_{x}^{\prime}$ is surjective for all but finitely many $x \in X_{0}$. We prove this in the following lemma.

LEMMA 5.7. (a) The map $Q_{x}$ is surjective for all $x \in X_{0}$.

(b) The map $Q_{x}^{\prime}$ is surjective for all but finitely many $x \in X_{0}$.

Proof. (a) It is a standard fact that if $R$ is any semilocal ring, the kernel of the natural surjection $R^{*} \rightarrow K_{1}(R)$ consists of products of elements of the form $(1+c d)(1+d c)^{-1}$, where $c, d \in R$ and $1+c d, 1+d c \in R^{*}$ (see [Si], Chap. 6, Prop. 53). Now for any $x \in X_{0}$, suppose that $u, v \in B_{\hat{x}}$ with $1+u v, 1+v u \in B_{\hat{x}}^{*}$, and let $U, V \in A_{\hat{x}}$ be lifts via the quotient map $A_{\hat{x}} \rightarrow B_{\hat{x}}$ of $u$ and $v$, respectively. Then, since $\mathcal{R}_{\hat{x}}$ is nilpotent, it is easy to show that $1+U V$ and $1+V U$ belong to $A_{\hat{x}}^{*}$. This implies that the map $Q_{x}$ is surjective for all $x \in X_{0}$.

(b) For each $x \in X_{0}$, set $S K_{1}\left(\mathcal{B}_{\hat{x}}\right):=\operatorname{Ker}\left(l_{x}^{s s}\right)$. We claim that $S K_{1}\left(\mathcal{B}_{\hat{x}}\right)=0$ for all but finitely many $x \in X_{0}$. To show this, observe that since $\mathcal{A}$ is isotrivial, Lemma 1.8(b) implies that $B$ is semisimple. Let $U$ be any open affine subset of $X$. Then $\mathcal{B}(U)$ is an $\mathcal{O}(U)$-maximal order in $B$, and $\mathcal{B}(U)_{\hat{x}}=\mathcal{B}_{\hat{x}}$ for all $x \in U$. It therefore follows from a theorem of Oliver (see [C, R2], (45.17)) that $S K_{1}\left(\mathcal{B}_{\hat{x}}\right)=$ $S K_{1}\left(\mathcal{B}(U)_{\hat{x}}\right)=0$ for all but finitely many $x \in U$. This establishes the claim.

Suppose now that $x \in X_{0}$ is such that $S K_{1}\left(\mathcal{B}_{\hat{x}}\right)=0$, and let $b_{x} \in \operatorname{Ker}\left(\varphi_{x}^{s s} \mid \mathcal{B}_{\hat{x}}^{*}\right)$. Then, since $l_{x}^{s s}$ is injective, it follows from the commutative diagram

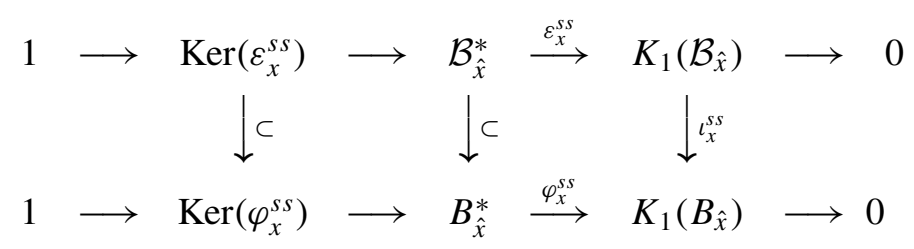


that $b_{x} \in \operatorname{Ker}\left(\varepsilon_{x}^{s s}\right)$. But now via an argument similar to that given above in part (a), it follows that we have $b_{x}=Q_{x}^{\prime}\left(a_{x}\right)$ for some $a_{x} \in \operatorname{Ker}\left(\varepsilon_{x}\right) \subseteq \mathcal{A}_{\hat{x}}^{*}$. Hence $Q_{x}^{\prime}$ is surjective for all $x \in X_{0}$ with $S K_{1}\left(\mathcal{B}_{\hat{x}}\right)=0$, that is $Q_{x}^{\prime}$ is surjective for all but finitely many $x \in X_{0}$.

This completes the proof of Corollary 5.5.

COROLLARY 5.8. Let $X$ be a curve, and $\mathcal{A}$ a sheaf of orders on $X$. If $\mathcal{A}_{\text {ss }}$ is also a sheaf of orders, then the semisimplification homomorphism $\sigma_{\mathcal{A}}$ is surjective.

Proof. Let $\sigma_{\mathcal{A}}^{\prime}$ denote the restriction of $\sigma_{\mathcal{A}}$ to $\mathrm{Cl}^{l f}(\mathcal{A})$. The commutative diagram used in the proof of Lemma 4.1 shows that $\sigma_{\mathcal{A}}$ is surjective if and only if $\sigma_{\mathcal{A}}^{\prime}$ has image $\mathrm{Cl}^{l f}\left(\mathcal{A}_{s s}\right)$. Set $\mathcal{D}:=\mathcal{A}_{s s}$, and write $D$ for the generic stalk of $\mathcal{D}$. Now, from Theorem 5.2 we obtain surjections $J\left(K_{1}(A)\right) \rightarrow \mathrm{Cl}^{l f}(\mathcal{A})$ and $J\left(K_{1}(D)\right) \rightarrow$ $\mathrm{Cl}^{l f}(\mathcal{D})$. By composing these with the natural surjections $J(A) \rightarrow J\left(K_{1}(A)\right)$ and $J(D) \rightarrow J\left(K_{1}(D)\right)$ which result from the fact that the rings $A_{\hat{x}}, \mathcal{A}_{\hat{x}}, D_{\hat{x}}$ and $\mathcal{D}_{\hat{x}}$ are semi-local for all $x \in X_{0}$ (cf. [C, R2], (40.31)) we obtain surjections $\mu: J(A) \rightarrow$ $\mathrm{Cl}^{l f}(\mathcal{A})$ and $\mu_{s s}: J(D) \rightarrow \mathrm{Cl}^{l f}(\mathcal{D})$. In addition, if $q: J(A) \rightarrow J(D)$ denotes the natural quotient map on ideles, then the following diagram commutes

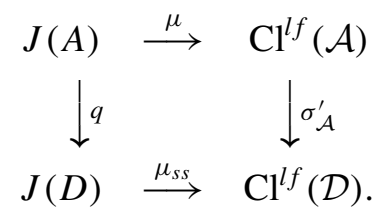

For each $x \in X_{0}, \mathcal{R}_{\hat{x}}$ is nilpotent (see Lemma 1.4(c)) and so the quotient maps $A_{\hat{x}}^{*} \rightarrow D_{\hat{x}}^{*}$ and $\mathcal{A}_{\hat{x}}^{*} \rightarrow \mathcal{D}_{\hat{x}}^{*}$ are surjective. Hence the map $q$ is surjective, and so it follows that $\sigma_{\mathcal{A}}^{\prime}$ is also surjective.

THEOREM 5.9. Let $X$ be a curve of genus $g$, and $\mathcal{A}$ an isotrivial sheaf of orders on $X$. Suppose that $t$ is a natural number.

(a) The group $D^{s s}(\mathcal{A})$ is finite, and

$$
\# D^{s s}(\mathcal{A}) \leqslant(\# k)^{g \cdot \operatorname{dim}_{k}(\mathcal{R}(X))} .
$$

Hence $D_{t}^{s s}(\mathcal{A})$ is also finite.

(b) Suppose that $\mathcal{A}_{s s}^{(t)}$ is commutative. Then

$$
\# D_{t}^{s s}(\mathcal{A}) \leqslant(\# k)^{g \cdot \operatorname{dim}_{k}\left(\mathcal{R}(X)^{t}\right)},
$$

with equality if $\mathcal{A}$ is commutative.

Proof. Set $\mathcal{B}:=\mathcal{A}_{s s}^{(t)}$ and $\mathcal{R}:=\mathcal{R}(\mathcal{A})$. Since $\mathcal{A}$ is isotrivial, Lemma 1.8(a) implies that $\mathcal{B}$ is an isotrivial sheaf of orders. We consider the following commutative diagram of pointed sets: 


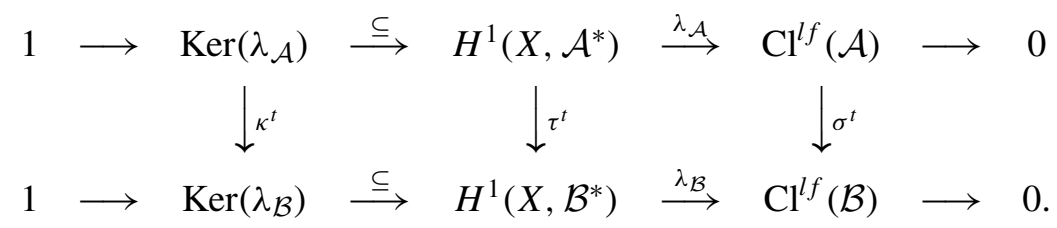

In this diagram $\lambda_{\mathcal{A}}$ and $\lambda_{\mathcal{B}}$ denote the surjective maps of Corollary 5.4, and so the rows are short exact sequences. We write $\sigma^{t}$ for the restriction of $\sigma_{\mathcal{A}}^{t}$ to $\mathrm{Cl}^{l f}(\mathcal{A})$, $\tau^{t}$ for the map induced by the quotient map $\mathcal{A}^{*} \rightarrow \mathcal{B}^{*}$ and $\kappa^{t}$ for the restriction of $\tau^{t}$ to $\operatorname{Ker}\left(\lambda_{\mathcal{A}}\right)$. The right-hand square of the diagram commutes and so $\operatorname{Im}\left(\kappa^{t}\right) \subseteq$ $\operatorname{Ker}\left(\lambda_{\mathcal{B}}\right)$ as indicated in the diagram. By applying the Snake lemma to the diagram one obtains an exact sequence (of pointed sets):

$$
1 \rightarrow \operatorname{Ker}\left(\kappa^{t}\right) \rightarrow \operatorname{Ker}\left(\tau^{t}\right) \rightarrow \operatorname{Ker}\left(\sigma^{t}\right) \rightarrow \operatorname{Im}\left(\kappa^{t}\right) \backslash \operatorname{Ker}\left(\lambda_{\mathcal{B}}\right) .
$$

Lemma 4.1 implies that $\operatorname{Ker}\left(\sigma^{t}\right)=D_{t}^{s s}(\mathcal{A})$ and, since $\mathcal{A}$ is isotrivial, Lemma 4.2 implies that $\operatorname{Ker}\left(\tau^{t}\right)$ corresponds bijectively with $H^{1}\left(X, 1+\mathcal{R}^{t}\right)$.

If $t=1$, then Corollary 5.5 implies that $\operatorname{Im}\left(\kappa^{t}\right)=\operatorname{Ker}\left(\lambda_{\mathcal{B}}\right)$, and so the above sequence gives rise to a surjective map

$$
H^{1}(X, 1+\mathcal{R}) \rightarrow D^{s s}(\mathcal{A}) .
$$

On the other hand, for any value of $t$, if $\mathcal{B}$ (respectively $\mathcal{A}$ ) is commutative, then Proposition 3.9 implies that $\operatorname{Ker}\left(\lambda_{\mathcal{B}}\right)$ is trivial (respectively that both $\operatorname{Ker}\left(\lambda_{\mathcal{A}}\right)$ and $\operatorname{Ker}\left(\lambda_{\mathcal{B}}\right)$ are trivial). Hence the above exact sequence implies that there is a surjective (respectively bijective) map

$$
H^{1}\left(X, 1+\mathcal{R}^{t}\right) \rightarrow D_{t}^{s s}(\mathcal{A}) .
$$

Thus, in order to prove both parts of the theorem, it suffices to show that

$$
\# H^{1}\left(X, 1+\mathcal{R}^{t}\right)=(\# k)^{g \cdot \operatorname{dim}_{k}\left(\mathcal{R}(X)^{t}\right)}
$$

for all integers $t \geqslant 0$ and for all isotrivial orders $\mathcal{A}$.

Let $e$ denote the smallest non-negative integer such that $\mathcal{R}^{e}$ is zero (see Lemma $1.4(c))$. Since $X$ is a curve we have that $H^{2}(X, \mathcal{V})=\{0\}$ for any sheaf $\mathcal{V}$. In particular therefore, for any natural numbers $a, b$ with $b \leqslant a \leqslant e-1$, the sequence (4.2) gives a short exact sequence (of finite pointed sets)

$$
0 \rightarrow H^{1}(X, \mathcal{Q}(a, a+1)) \rightarrow H^{1}(X, \mathcal{Q}(b, a+1)) \rightarrow H^{1}(X, \mathcal{Q}(b, a)) \rightarrow 0,
$$

and hence an equality

$$
\# H^{1}(X, \mathcal{Q}(b, a+1))=\left[\# H^{1}(X, \mathcal{Q}(a, a+1))\right]\left[\# H^{1}(X, \mathcal{Q}(b, a))\right] .
$$

Taking the product over these equalities with $b=t$ as $a$ runs from $t$ to $e-1$ (and noting that $\mathcal{Q}(t, e)=1+\mathcal{R}^{t}$ ) gives

$$
\# H^{1}\left(X, 1+\mathcal{R}^{t}\right)=\prod_{a=t}^{e-1} \# H^{1}(X, \mathcal{Q}(a, a+1)) .
$$


Since $\mathcal{A}$ is isotrivial, the isomorphism (4.4) implies that

$$
\# H^{1}(X, \mathcal{Q}(a, a+1))=\left[\# H^{1}(X, \mathcal{O})\right]^{\operatorname{dim}_{k}\left(\mathcal{R}(X)^{a} / \mathcal{R}(X)^{a+1}\right)}
$$

for each $a$ with $1 \leqslant a \leqslant e-1$. Since also

$$
\sum_{a=t}^{e-1} \operatorname{dim}_{k}\left(\mathcal{R}(X)^{a} / \mathcal{R}(X)^{a+1}\right)=\operatorname{dim}_{k}\left(\mathcal{R}(X)^{t}\right),
$$

this implies that

$$
\prod_{a=t}^{e-1} \# H^{1}(X, \mathcal{Q}(a, a+1))=\left[\# H^{1}(X, \mathcal{O})\right]^{\operatorname{dim}_{k}\left(\mathcal{R}(X)^{t}\right)} .
$$

The desired result now follows from the fact that (by our standing assumption) $k=\mathcal{O}(X)$ so that $\# H^{1}(X, \mathcal{O})=(\# k)^{g}$.

Remark 5.10. In the case that $\mathcal{A}=\mathcal{O}[G]$ with $\# G=p$, Theorem 5.9(b) with $t=1$ was first obtained by T. Chinburg (private communication).

To conclude this section, we now prove Theorem 4.8.

The isomorphism $\operatorname{det}_{\mathcal{A}}(-): \mathrm{Cl}^{l f}(\mathcal{A}) \rightarrow \operatorname{Pic}(\mathcal{A})$ of Proposition 3.9 has as inverse the first Chern class $c_{\mathcal{A}}$ : $\operatorname{Pic}(\mathcal{A}) \rightarrow K_{0}^{l f}(\mathcal{A})$ which is defined by sending the class $(\mathcal{L})$ to the element $(\mathcal{L})-(\mathcal{A})$ for each $\mathcal{L} \in \mathfrak{B}_{1}(\mathcal{A})$. We shall prove Theorem 4.8 by using the Riemann-Roch theorem and the bijectivity of such maps $c_{\mathcal{A}}$.

Let $k^{\prime}$ be an arbitrary finite extension of $k$ in $k^{c}$. Set $X^{\prime}:=X \times_{k} k^{\prime}$ and $\mathcal{O}^{\prime}:=\mathcal{O}_{X^{\prime}}$. Theorem 5.9(a) implies that $\# D^{s s}(\mathcal{A}) \leqslant(\# k)^{g \cdot d \operatorname{dim}_{k}(\mathcal{R}(X))}$, and so Theorem 4.8 will follow as a consequence of Proposition 4.6 if each section functor kernel of the form $D^{s f}\left(\mathcal{O}^{\prime}\right)$ may be identified with a subgroup of $\operatorname{Pic}^{0}(X)\left(k^{\prime}\right)$. Now if $\mathfrak{c}$ is any element of $K_{0}\left(X^{\prime}\right)$ such that $\chi R \Gamma_{\mathcal{O}^{\prime}, U}(\mathfrak{c})=0$ for some $U \in \mathcal{U}^{\text {aff }}\left(X^{\prime}\right)$, then $\operatorname{rank}(\mathfrak{c})=0$, and so $\mathfrak{c} \in \mathrm{Cl}^{l f}\left(\mathcal{O}^{\prime}\right)$. Hence $\mathfrak{c}$ lies in the image of the injection $c_{\mathcal{O}^{\prime}}: \operatorname{Pic}\left(X^{\prime}\right) \rightarrow K_{0}\left(X^{\prime}\right)$. But $\mathcal{O}^{\prime}\left(X^{\prime}\right)=k^{\prime}$ so that $K_{0}\left(\mathcal{O}^{\prime}\left(X^{\prime}\right)\right) \cong \mathbb{Z}$, and the RiemannRoch Theorem implies that if $\mathcal{L} \in \mathfrak{B}_{1}\left(\mathcal{O}^{\prime}\right)$, then the composite map

$$
\operatorname{Pic}\left(X^{\prime}\right) \stackrel{c_{\mathcal{O}^{\prime}}}{\rightarrow} K_{0}\left(X^{\prime}\right) \stackrel{\chi R \Gamma_{\mathcal{O}^{\prime}, X^{\prime}}}{\rightarrow} K_{0}\left(\mathcal{O}^{\prime}\left(X^{\prime}\right)\right) \cong \mathbb{Z}
$$

sends $(\mathcal{L})$ to $\operatorname{deg}_{X^{\prime}}(\mathcal{L})(\mathrm{cf}$. $[\mathrm{H}]$, Chap. $4, \S 1)$. Hence $D^{s f}\left(\mathcal{O}^{\prime}\right)$ may be identified with a subgroup of $\operatorname{Pic}^{0}\left(X^{\prime}\right)=\operatorname{Pic}^{0}(X)\left(k^{\prime}\right)$ as required.

\section{THE SEMISIMPLE CASE}

In this section we investigate $\mathrm{Cl}^{l f}(\mathcal{O}[G])$ in the case that $X$ is a curve, and $p$ does not divide \# $G$. The study of $\mathcal{O}[G]$-bundles in this case was initiated by R. Chapman in [Cha] and the results given here amplify those of loc. cit. 
We let $R_{G}$ denote the ring of $k^{c}$-valued characters of $G$, and we set $X^{c}:=$ $X \times_{k} k^{c}$. If $k[G] \cong \prod_{i \in I} M_{n(i)}\left(k_{m(i)}\right)$ is the Wedderburn decomposition of $k[G]$, then

$$
\begin{aligned}
\mathcal{O}[G] & \cong \prod_{i \in I} \mathcal{O} \otimes_{k} M_{n(i)}\left(k_{m(i)}\right) \\
& \cong \prod_{i \in I} M_{n(i)}\left(\left(f_{m(i)}\right)_{*} \mathcal{O}_{X_{m(i)}}\right)
\end{aligned}
$$

and so

$$
\begin{aligned}
\mathrm{Cl}^{l f}(\mathcal{O}[G]) & \cong \bigoplus_{i \in I} \mathrm{Cl}^{l f}\left(M_{n(i)}\left(\left(f_{m(i)}\right)_{*} \mathcal{O}_{X_{m(i)}}\right)\right) \\
& \cong \bigoplus_{i \in I} \mathrm{Cl}^{l f}\left(\left(f_{m(i)}\right)_{*} \mathcal{O}_{X_{m(i)}}\right)
\end{aligned}
$$

(Proposition 3.8(b) and Examples 3.4)

$$
\cong \bigoplus_{i \in I} \operatorname{Pic}\left(X_{m(i)}\right)
$$

(Proposition 3.9 and $\left.\operatorname{Spec}\left(\left(f_{m(i)}\right)_{*} \mathcal{O}_{X_{m(i)}}\right) \cong X_{m(i)}\right)$

$$
\begin{aligned}
& \cong \bigoplus_{i \in I} \operatorname{Hom}_{\Omega_{k}}\left(\mathbb{Z}\left[\operatorname{Gal}\left(k_{m(i)} / k\right)\right], \operatorname{Pic}\left(X^{c}\right)\right) \quad(\text { Lemma 4.9) } \\
& \cong \operatorname{Hom}_{\Omega_{k}}\left(\bigoplus_{i \in I} \mathbb{Z}\left[\operatorname{Gal}\left(k_{m(i)} / k\right)\right], \operatorname{Pic}\left(X^{c}\right)\right)
\end{aligned}
$$

If $\operatorname{Ir}_{G}$ denotes the set of irreducible $k^{c}$-valued characters of $G$, then $\operatorname{Ir}_{G}$ is a disjoint union of $\Omega_{k}$-conjugacy classes $\left\{\operatorname{Ir}_{G, i}: i \in I\right\}$ where, for each $i \in I$, the field $k_{m(i)}$ is generated over $k$ by the values of any (and therefore every) character which belongs to $\operatorname{Ir}_{G, i}$. For each $i \in I$ we fix a character $\chi_{i} \in \operatorname{Ir}_{G, i}$ and hence an $\Omega_{k}$-equivariant bijection between $\operatorname{Ir}_{G, i}$ and $\operatorname{Gal}\left(k_{m(i)} / k\right.$ ) (so that $\chi_{i}$ corresponds to the identity element of $\left.\mathrm{Gal}\left(k_{m(i)} / k\right)\right)$. These bijections induce an isomorphism

$$
\bigoplus_{i \in I} \mathbb{Z}\left[\operatorname{Gal}\left(k_{m(i)} / k\right)\right] \cong \bigoplus_{i \in I} \mathbb{Z}\left[\operatorname{Ir}_{G, i}\right]=R_{G}
$$

and by combining this with (6.2) we obtain an isomorphism

$$
\mathrm{Cl}^{l f}(\mathcal{O}[G]) \cong \operatorname{Hom}_{\Omega_{k}}\left(R_{G}, \operatorname{Pic}\left(X^{c}\right)\right) .
$$

Our aim in this section is to make the isomorphism (6.4) amenable to explicit computation. To do this we use the generalised resolvent formalism of Fröhlich as adapted by Chapman (cf. [Fr], [Cha]). Set $K^{c}:=K \otimes_{k} k^{c}$. It follows from Corollary 5.4 that we need only describe those elements of $\operatorname{Hom}_{\Omega_{k}}\left(R_{G}, \operatorname{Pic}\left(X^{c}\right)\right)$ which correspond to $\mathcal{O}[G]$-bundles $\mathcal{V}$ of rank one. In this case the stalk $\mathcal{V}_{x}$ is freely generated over $\mathcal{O}_{x}[G]$ by an element $v_{x}$, say, for each $x \in X$. Since each point $x \in X_{0}$ is a specialisation of the unique generic point $\eta$ of $X$, there is a natural 
injective map from each $\mathcal{V}_{x}$ to the generic stalk $\mathcal{V}_{\eta}$. In this way each stalk $\mathcal{V}_{x}$ can be regarded as an $\mathcal{O}_{x}[G]$-lattice in the free $K[G]$-module $\mathcal{V}_{\eta}$, so that $v_{x}=\lambda_{x} . v_{\eta}$ for a unique element $\lambda_{x}$ of $K[G]^{*}$. For each natural number $s$ we let $G L_{s}\left(K^{c}\right)$ denote the unit group of the ring $M_{s}\left(K^{c}\right)$. We extend each representation $T: G \longrightarrow G L_{s}\left(K^{c}\right)$ of $G$ to a $K^{c}$-algebra homomorphism $\tilde{T}: K^{c}[G] \longrightarrow M_{s}\left(K^{c}\right)$ in the obvious way, and we then define

$$
D(\mathcal{V})_{\chi}:=\sum_{y \in\left(X^{c}\right)_{0}} \operatorname{Val}_{y}\left(\operatorname{det}\left(\tilde{T}\left(\lambda_{x(y)}\right)\right)\right) y,
$$

where here $\chi$ is the character of $T, \operatorname{Val}_{y}$ denotes the valuation of $K^{c}$ corresponding to $y$, and $x(y) \in X_{0}$ is the point which lies beneath $y$. It is not difficult to see that the formal sum $D(\mathcal{V})_{\chi}$ belongs to $\operatorname{Div}\left(X^{c}\right)$, and is independent of the choice of representation $T$ which affords $\chi$. Write $\left[D(\mathcal{V})_{\chi}\right]$ for the image of $D(\mathcal{V})_{\chi}$ in $\operatorname{Pic}\left(X^{c}\right)$. Then $\left[D(\mathcal{V})_{\chi}\right]$ is independent of the choice of each element $\lambda_{x(y)}$ as described above, and the map $[\mathcal{V}] \in \operatorname{Hom}\left(R_{G}, \operatorname{Pic}\left(X^{c}\right)\right)$ which is induced by sending each character $\chi$ to the class $\left[D(\mathcal{V})_{\chi}\right]$ actually belongs to $\operatorname{Hom}_{\Omega_{k}}\left(R_{G}, \operatorname{Pic}\left(X^{c}\right)\right)$ (cf. [Cha], Lem. 1, Lem. 2 and Prop. 1).

PROPOSITION 6.1. If $p$ does not divide \# $G$, then the map

$$
\theta_{\mathcal{O}[G]}: \mathrm{Cl}^{l f}(\mathcal{O}[G]) \longrightarrow \operatorname{Hom}_{\Omega_{k}}\left(R_{G}, \operatorname{Pic}\left(X^{c}\right)\right)
$$

which is induced by sending each $\mathcal{O}[G]$-bundle $\mathcal{L}$ of rank one to the element $[\mathcal{L}]$ defined above is an isomorphism.

Proof. It suffices to prove that for each $\mathcal{O}[G]$-bundle $\mathcal{L}$ of rank one the isomorphism (6.4) sends the class of $\mathcal{L}$ to the homomorphism $[\mathcal{L}]$. To do this it is in turn enough to replace $\mathcal{O}[G]$ by each of its components in the decomposition (6.1). We thus fix an index $i \in I$, set $\mathcal{C}:=M_{n(i)}\left(\left(f_{m(i)}\right)_{*} \mathcal{O}_{X_{m(i)}}\right)$ and let $C$ denote the corresponding component of $K[G]$. We set $n:=n(i), k^{\prime}:=k_{m(i)}, X^{\prime}:=X \times_{k} k^{\prime}$, $L:=k\left(X^{\prime}\right)$ and let $f^{\prime}: X^{\prime} \rightarrow X$ denote the natural morphism. For any indecomposable idempotent $e$ of $M_{n}\left(k^{\prime}\right)$ the $n$-dimensional $k^{\prime}$-space $M_{n}\left(k^{\prime}\right) e$ affords the character $\chi_{i}$. If $T: G \rightarrow G L_{n}\left(k^{\prime}\right)$ is the corresponding representation of $G$, then the restriction to $C$ of the associated algebra morphism $\tilde{T}: K[G] \rightarrow M_{n}(L)$ is the identity map. Let $\mathcal{C}^{\prime}$ denote the sheaf of orders $f_{*}^{\prime} \mathcal{O}_{X^{\prime}}$. Then the isomorphism of Proposition 3.8(b) sends $(\mathcal{L}) \in \mathrm{Cl}^{l f}(\mathcal{C})$ to the class of the $\mathcal{C}^{\prime}$-bundle $\mathcal{L}^{\prime}$ which has sections $\mathcal{L}^{\prime}(U)=e M_{n}\left(k^{\prime}\right) \otimes_{M_{n}\left(k^{\prime}\right)} \mathcal{L}(U)=e \mathcal{L}(U)$ for each open subset $U$ of $X$. The isomorphism $\mathrm{Cl}^{l f}\left(\mathcal{C}^{\prime}\right) \cong \operatorname{Pic}\left(\mathcal{C}^{\prime}\right)$ of Proposition 3.9 sends the class of $\mathcal{L}^{\prime}$ to the class of $\operatorname{det}_{\mathcal{C}^{\prime}} \mathcal{L}^{\prime}$.

We now view $\mathcal{L}$ as a subsheaf of the constant sheaf $M_{n}(L)$, and we choose an affine open covering $\mathcal{U}=\left\{U_{s}\right\}$ of $X$ such that, for each index $s, \mathcal{L}\left(U_{s}\right)=M_{n}\left(\mathcal{C}^{\prime}\left(U_{s}\right)\right) a_{s}$ for some element $a_{s} \in G L_{n}(L)$. The space of sections of $\operatorname{det}_{\mathcal{C}^{\prime}} \mathcal{L}^{\prime}$ over $U_{s}$ is then equal to $\mathcal{C}^{\prime}\left(U_{s}\right) \operatorname{det}\left(a_{s}\right)$. For each $y \in\left(X^{\prime}\right)_{0}$ we choose $U_{s(y)} \in \mathcal{U}$ which contains $f^{\prime}(y)$ and we note that the valuation $\operatorname{Val}_{y}\left(\operatorname{det}\left(a_{s(y)}\right)\right)$ is independent of precisely which open set $U_{s(y)}$ and basis element $a_{s(y)}$ as above are chosen. Under the isomorphism $\operatorname{Pic}\left(\mathcal{C}^{\prime}\right) \cong \operatorname{Pic}\left(X^{\prime}\right)$ coming from Proposition 3.9, the class of $\operatorname{det}_{\mathcal{C}^{\prime}} \mathcal{L}^{\prime}$ 
is sent to the class of the divisor $\sum_{y \in\left(X^{\prime}\right)_{0}} \operatorname{Val}_{y}\left(\operatorname{det}\left(a_{s(y)}\right)\right) y$. The desired result thus follows from the fact that under the isomorphism $\operatorname{Pic}\left(X^{\prime}\right) \cong \operatorname{Hom}_{\Omega_{k}}\left(\mathbb{Z}\left[\operatorname{Ir}_{G, i}\right]\right.$, $\left.\operatorname{Pic}\left(X^{c}\right)\right)$ coming from Lemma 4.9 and (6.3) the class of $\sum_{y \in\left(X^{\prime}\right)_{0}} \operatorname{Val}_{y}\left(\operatorname{det}\left(a_{s(y)}\right)\right) y$ is sent to the homomorphism which is obtained by restricting $[\mathcal{L}]$ to $\mathbb{Z}\left[\operatorname{Ir}_{G, i}\right]$.

Remark 6.2. In [Cha] Chapman introduces the homomorphism $[\mathcal{L}]$ defined above as an important structural invariant of the rank one $\mathcal{O}[G]$-bundle $\mathcal{L}$. Proposition 6.1 verifies that $[\mathcal{L}]$ is in fact a natural representative of the class of $\mathcal{L}$ in $\mathrm{Cl}^{l f}(\mathcal{O}[G])$.

This 'Hom-description' of Fröhlich and Chapman turns out to be very well adapted to the explicit computation of those classes which arise from structure sheaves of tame Galois covers of $X$ (cf. [Cha] and [A, Bu]). As the next result shows, it is also amenable to studying functorial behaviour. We point out that such explicit descriptions of functoriality are often very useful in arithmetical applications.

PROPOSITION 6.3. Suppose that $X$ is a curve and that $G$ is a finite group such that $p$ does not divide \#G. Let $H$ be a subgroup of $G$.

(a) Let

$$
\operatorname{Ind}_{H}^{G}: \mathrm{Cl}^{l f}(\mathcal{O}[H]) \rightarrow \mathrm{Cl}^{l f}(\mathcal{O}[G])
$$

denote the map which is induced by the functor $\mathcal{O}[G] \otimes_{\mathcal{O}[H]}-$. Then there is a commutative diagram:

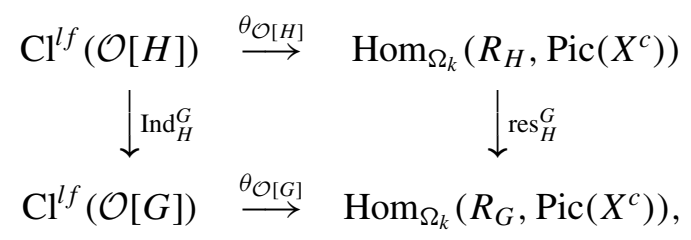

where the right-hand vertical arrow is induced by the restriction map $\operatorname{res}_{H}^{G}: R_{G} \rightarrow$ $R_{H}$ on characters.

(b) Let

$$
\operatorname{Res}_{H}^{G}: \mathrm{Cl}^{l f}(\mathcal{O}[G]) \rightarrow \mathrm{Cl}^{l f}(\mathcal{O}[H])
$$

denote the map which is induced by viewing an $\mathcal{O}[G]$-bundle as an $\mathcal{O}[H]$-bundle. Then there is a commutative diagram:

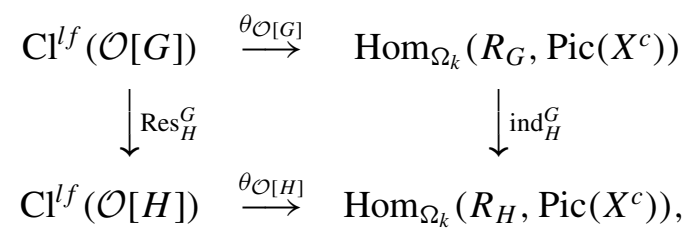


where the right-hand vertical arrow is induced by the induction map $\operatorname{ind}_{H}^{G}: R_{H} \rightarrow$ $R_{G}$ on characters.

(c) Suppose that $H$ is normal in G. Let

$$
\operatorname{Coinf}_{G / H}^{G}: \mathrm{Cl}^{l f}(\mathcal{O}[G]) \rightarrow \mathrm{Cl}^{l f}(\mathcal{O}[G / H])
$$

denote the map which is induced by the functor $\mathcal{O}[G / H] \otimes_{\mathcal{O}[G]}-$. Then there is a commutative diagram:

$$
\begin{aligned}
& \mathrm{Cl}^{l f}(\mathcal{O}[G]) \quad \stackrel{\theta_{\mathcal{O}[G]}}{\longrightarrow} \quad \operatorname{Hom}_{\Omega_{k}}\left(R_{G}, \operatorname{Pic}\left(X^{c}\right)\right) \\
& \downarrow \operatorname{Coinf}_{G / H}^{G} \quad \downarrow_{\inf _{G / H}^{G}}^{G} \\
& \mathrm{Cl}^{l f}(\mathcal{O}[G / H]) \stackrel{\theta_{\mathcal{O}[G / H]}}{\longrightarrow} \operatorname{Hom}_{\Omega_{k}}\left(R_{G / H}, \operatorname{Pic}\left(X^{c}\right)\right),
\end{aligned}
$$

where the right-hand vertical arrow is induced by the inflation map $\inf _{G / H}^{G}$ : $R_{G / H} \rightarrow R_{G}$ on characters.

Proof. This is formally the same as the proof of ([Fr], Chap. II, Th. 12).

\section{GROUP RINGS OF ABELIAN GROUPS}

In this section, we assume that $X$ is any irreducible, projective variety over $k$. Let $G$ be any finite Abelian group. We shall explain how the group $\operatorname{Pic}(\mathcal{O}[G])$ and the filtration $\left\{D_{i}^{s s}(\mathcal{O}[G])\right\}_{i} \geqslant 0$ have interesting re-interpretations.

Let $\mathcal{P}$ denote the Picard scheme $\operatorname{Pic}_{X / k}$ of $X / k$, and suppose that $\mathcal{A}$ is any isotrivial sheaf of commutative orders on $X$ (e.g. $\mathcal{A}=\mathcal{O}[G]$ ). The following result, which was explained to us by G. Pappas, shows that the $\operatorname{group} \operatorname{Pic}(\mathcal{A})$ is isomorphic to the group of $\operatorname{Spec}(\mathcal{A}(X))$-valued points of $\mathcal{P}$. In this context, it is interesting to recall that if $X$ is a curve, then there is a canonical identification between $\operatorname{Pic}(\mathcal{A})$ and $\mathrm{Cl}^{l f}(\mathcal{A})$ (see Proposition 3.9).

PROPOSITION 7.1. There is a natural isomorphism $\operatorname{Pic}(\mathcal{A}) \simeq \mathcal{P}(\operatorname{Spec}(\mathcal{A}(X))$.

Proof. We set $S:=\operatorname{Spec}(\mathcal{A}(X))$ and $X_{S}:=X \times_{k} S$. As $\mathcal{A}$ is the structure sheaf of $X_{S}$, it follows that we may view rank one $\mathcal{A}$-bundles on $X$ as line bundles on $X_{S}$, and that $\operatorname{Pic}(\mathcal{A})$ is the group of isomorphism classes of line bundles on $X_{S}$ (see the proof of Sublemma 3.9.1, for example). Since all line bundles on $S$ are trivial, it follows immediately from the definition of the Picard scheme (see e.g. [M,F], Chap. 0, § 5) that $\operatorname{Pic}(\mathcal{A}) \simeq \mathcal{P}(S)$, as asserted.

In the following Corollary (which will be used in Section 9), we write $\boldsymbol{\mu}_{\boldsymbol{d}}$ for the subgroup scheme of $d$-torsion of the multiplicative group $\mathbb{G}_{m}$ over $k$.

COROLLARY 7.2. Suppose that $G$ is cyclic of order $d$. Then there is a natural isomorphism $\operatorname{Pic}(\mathcal{O}[G]) \simeq \mathcal{P}\left(\boldsymbol{\mu}_{\boldsymbol{d}}\right)$. 
Proof. Let $T$ be an indeterminate. Then $k[G] \simeq k[T] /\left(T^{d}-1\right)$, and so $\operatorname{Spec}(k[G])=\boldsymbol{\mu}_{\boldsymbol{d}}$. The result thus follows immediately from Proposition 7.1.

We now discuss the subgroups $\left\{D_{i}^{s s}(\mathcal{O}[G])\right\}_{i} \geqslant 0$ of $K_{0}^{l f}(\mathcal{O}[G])$. To do this we write $\# G=p^{n} r$ with $(p, r)=1$, and we let $P$ and $H$ denote the subgroups of $G$ which have orders $p^{n}$ and $r$, respectively. We set $\mathcal{R}:=\mathcal{R}(\mathcal{O}[G])$.

Since $G$ is the direct product of $H$ and $P$, there is an identification $\mathcal{O}[G]=$ $\mathcal{O}[H] \otimes_{k} k[P]$. With respect to this identification, one has $\mathcal{R}=\mathcal{O}[H] \otimes_{k} I(k[P])$ where $I(k[P])$ denotes the augmentation ideal of $k[P]$. This ideal is the kernel of the natural surjection $\mathcal{O}[G] \rightarrow \mathcal{O}[G / P] \cong \mathcal{O}[H]$ and so $\mathcal{O}[G]_{s s} \cong \mathcal{O}[H]$. The semisimplification homomorphism

$$
\sigma_{\mathcal{O}[G]}: \mathcal{O}[G] \rightarrow \mathcal{O}[H]
$$

is thus canonically split by the map from $K_{0}^{l f}(\mathcal{O}[H])$ to $K_{0}^{l f}(\mathcal{O}[G])$ which is induced by the functor $\mathcal{O}[G] \otimes_{\mathcal{O}[H]}-$.

For each integer $i$ with $0 \leqslant i \leqslant n$ we now write $P^{i}$ for the subgroup of $G$ which is generated by the $p^{i}$-th powers of elements of $P$. Then $\mathcal{R}^{p^{i}}$ is equal to the kernel $\mathcal{O}[H] \otimes_{k} I\left(k\left[P^{i}\right]\right) k[P]$ of the natural surjection $\mathcal{O}[G] \longrightarrow \mathcal{O}\left[G / P^{i}\right]$, and so $\mathcal{O}[G]_{s s}^{\left(p^{i}\right)} \cong \mathcal{O}\left[G / P^{i}\right]$.

We write

$$
\operatorname{Coinf}_{G / P_{i}}^{G}: K_{0}^{l f}(\mathcal{O}[G]) \rightarrow K_{0}^{l f}\left(\mathcal{O}\left[G / P^{i}\right]\right)
$$

for the co-inflation homomorphism which is induced by the functor $\mathcal{O}\left[G / P^{i}\right] \otimes_{\mathcal{O}[G]}-$. The following result is now clear.

LEMMA 7.3. For each integer $i$ with $0 \leqslant i \leqslant n$ one has

$$
D_{p^{i}}^{s s}(\mathcal{O}[G])=\operatorname{Ker}\left(\operatorname{Coinf}_{G / P_{i}}^{G}: K_{0}^{l f}(\mathcal{O}[G]) \rightarrow K_{0}^{l f}\left(\mathcal{O}\left[G / P^{i}\right]\right)\right) .
$$

Lastly we observe that Lemma 4.2 can also be reinterpreted in this case. Indeed, for each integer $i$ with $0 \leqslant i<n$ one has a short exact sequence of sheaves

$$
0 \rightarrow \mathcal{R}^{p^{i}} / \mathcal{R}^{p^{i+1}} \rightarrow\left(\mathcal{O}[G]_{s s}^{\left(p^{i+1}\right)}\right)^{*} \rightarrow\left(\mathcal{O}[G]_{s s}^{\left(p^{i}\right)}\right)^{*} \rightarrow 0
$$

in which the second arrow is induced by the truncated exponential map $x \mapsto$ $\sum_{s=0}^{s=p-1} x^{s} / s$ ! and the third arrow is the natural quotient map. By taking sheaf cohomology of these sequences one can recover the relevant parts of Lemma 4.2.

\section{Cyclic Groups of $p$-Power Order}

In the last two sections of this paper, we shall restrict ourselves to the case in which $\mathcal{A}=\mathcal{O}[G]$ with $G$ a cyclic group of $p$-power order. We write $\mathbb{F}_{p}$ for the finite field of $p$ elements. 


\section{WITT VECTORS}

In this section, we allow $X$ to be any irreducible scheme over $k$, and we let $G$ denote a cyclic group of order $p^{n}$. We shall describe $\operatorname{Pic}(\mathcal{O}[G])$ in terms of the Zariski cohomology of sheaves of Witt vectors. In the next section we shall see that this description can be useful in arithmetical contexts.

For any $\mathbb{F}_{p}$-algebra $B$, we let $\mathrm{W}(B)$ denote the ring of Witt vectors with coefficients in $B$. For any natural number $s$ we write $\mathrm{W}_{s}(B)$ for the quotient of $\mathrm{W}(B)$ consisting of Witt vectors of length $s$. We write $V$, respectively $F$, for the Verschiebung, respectively Frobenius, map on $W(B)$. Thus, if $\left(b_{0}, b_{1}, \ldots\right) \in W(B)$, then $V\left(b_{0}, b_{1}, \ldots\right)=\left(0, b_{0}, b_{1}, \ldots\right)$ and $F\left(b_{0}, b_{1}, \ldots\right)=\left(b_{0}^{p}, b_{1}^{p}, \ldots\right)$. By passage to quotient one obtains induced maps

$$
V: \mathrm{W}_{s}(B) \rightarrow \mathrm{W}_{s+1}(B)
$$

and

$$
F: \mathrm{W}_{s}(B) \rightarrow \mathrm{W}_{s}(B) .
$$

If $s \geqslant t$ we write

$$
R_{s, t}: \mathrm{W}_{s}(B) \rightarrow \mathrm{W}_{t}(B)
$$

for the restriction map $\left(b_{0}, \ldots, b_{s-1}\right) \mapsto\left(b_{0}, \ldots, b_{t-1}\right)$.

We write $\mathcal{W}_{s}$ for the sheaf of Witt vectors of length $s$ on $X$ as introduced in [S1] (see also [S2], Chap. V). In what follows, we shall often abuse notation and write for example $V: H^{1}\left(X, \mathcal{W}_{s}\right) \rightarrow H^{1}\left(X, \mathcal{W}_{s+1}\right)$ for the morphism induced by the Verschiebung. This should not cause any confusion.

Let $T$ be an indeterminate, and fix a generator $g$ of $G$. For each affine open subset $U$ of $X$, we fix an identification of $\mathcal{A}(U)[G]^{*}$ with the group of units of the ring $R(U)_{n}:=\mathcal{A}(U)[T] /\left(T^{p^{n}}\right)$ by letting the class $t_{n}$ of $T$ in $R(U)_{n}$ correspond to the element $g-1 \in \mathcal{A}(U)[G]$. We write $E(-)$ for the Artin-Hasse exponential function on Witt vectors. For each integer $i>0$ we let $r_{i}(n)$ denote the least positive integer such that $p^{r_{i}(n)} \geqslant p^{n} / i$. We recall that each element $\alpha$ of $R(U)_{n}^{*}$ can be written uniquely as a product

$$
\alpha=a_{0} \times \prod_{\substack{0<i<p^{n} \\(i, p)=1}} E\left(a_{i} \cdot T^{i}\right)
$$

where here $a_{0} \in \mathcal{A}(U)^{*}$, each $a_{i}$ belongs to $\mathrm{W}_{r_{i}(n)}(\mathcal{A}(U))$ and $a_{i} \cdot T^{i}$ denotes the product in $\mathrm{W}_{r_{i}(n)}(\mathcal{A}(U)[T])$ of $a_{i}$ by $\left(T^{i}, 0,0, \ldots\right) \in \mathrm{W}_{r_{i}(n)}(\mathcal{A}(U)[T])$ (see [S2], Chap. V, Prop. 9). This decomposition in turn induces an isomorphism of sheaves of groups on $X$

$$
\mathcal{O}[G]^{*} \cong \mathcal{O}^{*} \times \prod_{\substack{0<i<p^{n} \\(i, p)=1}} \mathcal{W}_{r_{i}(n)} .
$$


Set $\mathrm{W}(\mathcal{O}[G])_{-1}:=\operatorname{Pic}(\mathcal{O})$ and $\mathrm{W}(\mathcal{O}[G])_{i}:=H^{1}\left(X, \mathcal{W}_{r_{i}(n)}\right)$ for each integer $i$ with $0<i<p^{n}$ and $(i, p)=1$. We define

$$
\mathrm{W}(\mathcal{O}[G]):=\bigoplus_{\substack{-1 \leqslant i \leqslant p^{n} \\(i, p)=1}} \mathrm{~W}(\mathcal{O}[G])_{i}
$$

In conjunction with the canonical identification $\operatorname{Pic}(\mathcal{O}[G]) \cong H^{1}\left(X, \mathcal{O}[G]^{*}\right)$ induced by Lemma 2.4 (with $n=1$ ), one obtains from the decomposition (8.2) an isomorphism (which depends upon the choice of generating element $g$ )

$$
\omega_{g}: \operatorname{Pic}(\mathcal{O}[G]) \cong \mathrm{W}(\mathcal{O}[G]) .
$$

In the next section, we shall see that this explicit isomorphism can be useful in the context of arithmetical computations.

Our next result is a natural analogue of Proposition 6.3 and it shows that the isomorphism (8.3) is also useful for discussing functorial behaviour. Before stating the result, we introduce the following piece of notation. Let $H$ be any cyclic group of $p$-power order, and let $\tau$ be any homomorphism with domain $\mathrm{W}(\mathcal{O}[H])$. Then, for each integer $i$ with $-1 \leqslant i \leqslant \# H$ and $(i, p)=1$, we write $\tau_{i}$ for the restriction of $\tau$ to the direct summand $\mathrm{W}(\mathcal{O}[H])_{i}$ of $\mathrm{W}(\mathcal{O}[H])$.

PROPOSITION 8.1. Let $H$ be the subgroup of $G$ of order $p^{j}$ (so that $0 \leqslant j \leqslant n$ ).

(a) Let

$$
V^{n-j}: \mathrm{W}(\mathcal{O}[H]) \rightarrow \mathrm{W}(\mathcal{O}[G])
$$

be the homomorphism defined by

$$
V_{-1}^{n-j}=\mathrm{id}: \mathrm{W}(\mathcal{O}[H])_{-1} \rightarrow \mathrm{W}(\mathcal{O}[G])_{-1},
$$

and

$$
V_{i}^{n-j}=V^{r_{i}(n)-r_{i}(j)}: \mathrm{W}(\mathcal{O}[H])_{i} \rightarrow \mathrm{W}(\mathcal{O}[G])_{i}
$$

for each integer $i$ with $0<i<p^{j}$ and $(i, p)=1$. If $h$ denotes $g^{p^{n-j}}$, then the following diagram commutes:

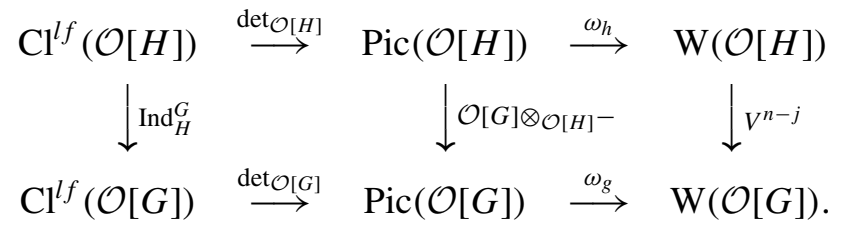

(b) Let

$$
R^{j}: \mathrm{W}(\mathcal{O}[G]) \rightarrow \mathrm{W}(\mathcal{O}[G / H])
$$


be the homomorphism defined by

$$
\begin{aligned}
& R_{-1}^{j}=\mathrm{id}: \mathrm{W}(\mathcal{O}[G])_{-1} \rightarrow \mathrm{W}(\mathcal{O}[G / H])_{-1}, \\
& R_{i}^{j}=R_{r_{i}(n), r_{i}(n-j)}: \mathrm{W}(\mathcal{O}[G])_{i} \rightarrow \mathrm{W}(\mathcal{O}[G / H])_{i}
\end{aligned}
$$

for each integer $i$ with $0<i<p^{n-j}$ and $(i, p)=1$, and

$$
R_{i}^{j}=0
$$

for each integer $i>p^{n-j}$. If $\tilde{g}$ denotes the image of $g$ in $G / H$, then the following diagram commutes:

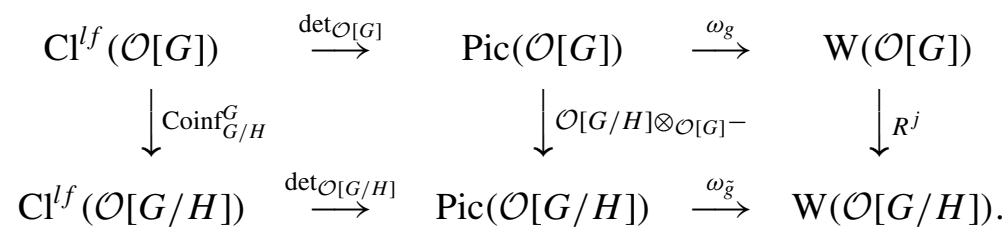

(c) Let

$$
\Pi^{n-j}: \mathrm{W}(\mathcal{O}[G]) \rightarrow \mathrm{W}(\mathcal{O}[H])
$$

be the homomorphism defined by

$$
\begin{aligned}
& \Pi_{-1}^{n-j}=p^{n-j}: \mathrm{W}(\mathcal{O}[G])_{-1} \rightarrow \mathrm{W}(\mathcal{O}[H])_{-1}, \\
& \Pi_{i}^{n-j}=F^{n-j} \circ R_{r_{i}(n), r_{i}(j)}: \mathrm{W}(\mathcal{O}[G])_{i} \rightarrow \mathrm{W}(\mathcal{O}[H])_{i}
\end{aligned}
$$

for each integer $i$ with $0<i<p^{j}$ and $(i, p)=1$, and

$$
\Pi_{j}^{n-j}=0
$$

for each integer $i>p^{j}$. If $h$ denotes $g^{p^{n-j}}$, then the following diagram commutes:

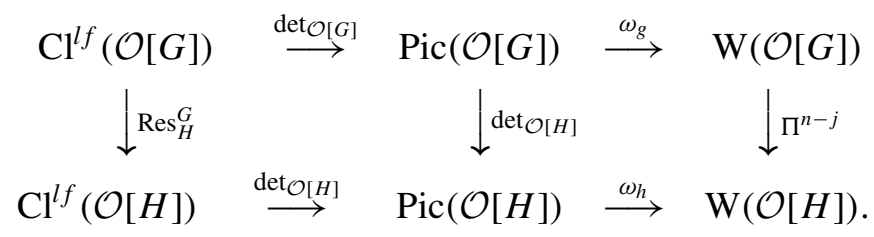

Proof. In each case the commutativity of the left hand square is a straightforward consequence of standard properties of the determinant map. Indeed, cases (a) and (b) follow easily from the fact that determinants commute with scalar extension, and in (c) one uses the following well known fact. After fixing a set of coset representatives of $H$ in $G$, one obtains for each commutative ring $\Lambda$ and each natural number $m$ a canonical identification $\iota_{m}: G L_{m}(\Lambda[G]) \cong G L_{m p^{n-j}}(\Lambda[H])$. Then for each $\theta \in G L_{m}(\Lambda[G])$ one has

$$
\operatorname{det}\left(\iota_{m}(\theta)\right)=\operatorname{det}\left(\iota_{1}(\operatorname{det}(\theta))\right) \in \Lambda[H]^{*} .
$$


It remains to discuss the commutativity of the right hand squares. To do this we let $U$ be any affine open subset of $X$.

The commutativity of the right hand square in (a) can be deduced from the following fact. We identify $R(U)_{j}$ with a subring of $R(U)_{n}$ so that $t_{j}$ corresponds to $t_{n}^{p^{n-j}}$. Then, for each integer $i$ with $0<i<p^{j}$ and $(i, p)=1$, and each element $a_{i}$ of $\mathrm{W}_{r_{i}(j)}(\mathcal{A}(U))$ one has

$$
E\left(a_{i} \cdot\left(T^{p^{n-j}}\right)^{i}\right)=E\left(V^{r_{i}(n)-r_{i}(j)} a_{i} \cdot T^{i}\right) \in R(U)_{n}^{*} .
$$

In case (b) the commutativity of the right-hand square is a consequence of the fact that for each integer $i$ with $0<i<p^{n}$ and $(i, p)=1$, and for each element $a_{i}$ of $\mathrm{W}_{r_{i}(n)}(\mathcal{A}(U))$, the projection of $E\left(a_{i} \cdot T^{i}\right) \in R(U)_{n}^{*}$ into $R(U)_{n-j}^{*}$ is equal to

$$
\begin{aligned}
& 1, \quad \text { if } i>p^{n-j}, \\
& E\left(R_{r_{i}(n), r_{i}(n-j)} a_{i} \cdot T^{i}\right), \quad \text { if } i \leqslant p^{n-j} .
\end{aligned}
$$

To deal with the right-hand square in case (c) we shall assume that $j=n-1$ (the general case follows from this by an induction on $n-j$ ). We set $\mathfrak{R}_{p}:=$ $\{0,1, \ldots, p-1\}$ and for each integer $i$ we let $\{i\}$ denote the unique element of $\mathfrak{R}_{p}$ such that $i-\{i\}$ is divisible by $p$. Each $\alpha \in \mathcal{A}(U)[G]^{*}$ induces an $\mathcal{A}(U)[H]$ automorphism of $\mathcal{A}(U)[G]$ and we let $\theta(\alpha)$ denote its matrix as computed with respect to the $\mathcal{A}(U)[H]$-basis $\left\{g^{i}: i \in \mathfrak{R}_{p}\right\}$ of $\mathcal{A}(U)[G]$. The commutativity of the right hand square in (c) (with $j=n-1$ ) follows easily from the equality $\operatorname{det}(\theta(\alpha))=\alpha^{p}$ and the fact that $p=F \circ V$ on $\mathrm{W}(B)$ for any $\mathbb{F}_{p}$-algebra $B$. In the remainder of the proof we shall explain why $\operatorname{det}(\theta(\alpha))=\alpha^{p}$. To do this, we define $h_{s} \in \mathcal{A}(U)[H]$ for each $s \in \Re_{p}$ by the equality $\alpha=\sum_{s \in \mathfrak{R}_{p}} g^{s} h_{s}$.

LEMMA 8.2. Let $\theta^{\prime}(\alpha)$ denote the $p \times p$ matrix with entries $\theta^{\prime}(\alpha)_{(i, j)}=g^{\{j-i\}} h_{\{j-i\}}$ for each $(i, j) \in \mathfrak{R}_{p} \times \mathfrak{R}_{p}$. Then $\operatorname{det}\left(\theta^{\prime}(\alpha)\right)=\operatorname{det}(\theta(\alpha))$.

Proof. The $(i, j)$ entry of $\theta(\alpha)$ is equal to $h_{\{j-i\}}$ if $j \geqslant i$ and to $g^{p} h_{\{j-i\}}$ if $j<i$ so that

$$
\operatorname{det}(\theta(\alpha))=\sum_{\sigma \in S_{n}} \operatorname{sign}(\sigma) g^{p t(\sigma)} \prod_{i \in \Re_{p}} h_{\{\sigma(i)-i\}}
$$

where $S_{n}$ is the group of permutations on $n$ symbols and $t(\sigma):=\#\left\{i \in \mathfrak{R}_{p}: \sigma(i)<\right.$ i) for each $\sigma \in S_{n}$. Since

$$
\operatorname{det}\left(\theta^{\prime}(\alpha)\right)=\sum_{\sigma \in S_{n}} \operatorname{sign}(\sigma) g^{\sum_{i \in \mathfrak{R}_{p}}\{\sigma(i)-i\}} \prod_{i \in \mathfrak{R}_{p}} h_{\{\sigma(i)-i\}},
$$

it therefore suffices to show that $p t(\sigma)=\sum_{i \in \mathfrak{R}_{p}}\{\sigma(i)-i\}$ for each $\sigma \in S_{n}$. However, since $\sum_{i \in \mathfrak{R}_{p}}(\sigma(i)-i)=0$ this last equality is easily verified.

LEMMA 8.3. $\operatorname{det}\left(\theta^{\prime}(\alpha)\right)=\alpha^{p}$. 
Proof. We set $\Lambda:=\mathrm{W}(\mathcal{A}(U))$, and we begin by observing that we may lift $g \in G$ to $\hat{g} \in \Lambda[G]$ in the obvious manner. We then choose lifts $\hat{h}_{s} \in \Lambda[G]$ of $h_{s}$ for each $s \in \mathfrak{R}_{p}$, and we define $\hat{\theta}^{\prime}(\alpha) \in M_{p}(\Lambda[G])$ by

$$
\left(\hat{\theta}^{\prime}(\alpha)\right)_{(i, j)}:=\hat{g}^{\{j-i\}} \hat{h}_{\{j-i\}} .
$$

This matrix $\hat{\theta}^{\prime}(\alpha)$ is then a lift of $\theta^{\prime}(\alpha)$ to $M_{p}(\Lambda[G])$.

We let $\Re_{p}^{+}$denote the set $\Re_{p}$ endowed with the group law $(i, j) \mapsto\{i+j\}$ for $i, j \in \Re_{p}$, and we set $\mathfrak{R}_{p}^{\vee}:=\operatorname{Hom}\left(\mathfrak{R}_{p}^{+}, \mu_{p}\right)$, where $\mu_{p}$ denotes the group of $p$-th roots of unity in an algebraic closure of the field of fractions of $W(k)$. Define a map $f: \mathfrak{R}_{p} \rightarrow \Lambda[G]$ by $f(i):=\hat{g}^{i} \hat{h}_{i}$ for each $i \in \mathfrak{R}_{p}$. Then we have

$$
\left(\hat{\theta}^{\prime}(\alpha)\right)_{(i, j)}=f(\{j-i\})
$$

for each $i$ and $j$. Since $\{j-i\}=j-i$ with respect to the group law on $\mathfrak{R}_{p}^{+}$it follows via a standard argument dealing with evaluation of determinants (see e.g. [Fr, T], App. A, § A13) that we therefore have

$$
\operatorname{det}\left(\hat{\theta}^{\prime}(\alpha)\right)=\prod_{\chi \in \Re_{p}^{\vee}}\left(\sum_{s \in \Re_{p}} \chi(s) \hat{g}^{s} \hat{h}_{s}\right) \in\left(\Lambda \otimes_{W(k)} W(k)\left[\mu_{p}\right]\right)[G] .
$$

Upon projecting this equality to $\mathcal{A}(U)[G]$ we obtain

$$
\operatorname{det}\left(\theta^{\prime}(\alpha)\right)=\prod_{\mathfrak{R}_{p}^{\vee}}\left(\sum_{s \in \Re_{p}} g^{s} h_{s}\right),
$$

and this is equal to $\alpha^{p}$ as required.

Taken together, Lemmas 8.2 and 8.3 complete the proof of Proposition 8.1.

\section{REALISABLE CLASSES}

Let $X$ be an irreducible, projective variety over $k$, and suppose that $G$ is a cyclic group of order $p^{n}$. In this section we shall apply the techniques discussed in Sections 7 and 8 to describe those elements of $\operatorname{Pic}(\mathcal{O}[G])$ which arise from structure sheaves of unramified $G$-covers $Y \rightarrow X$. The results in this section both clarify and extend results from ([A, Bu], § 2.1). In addition, Lemma 2.1 of [A, Bu] explains how some of the results of the present section extend easily to cover the case in which $G$ is an arbitrary finite Abelian $p$-group.

If $f: Y \rightarrow X$ is an unramified $G$-cover of $X$, then $f_{*} \mathcal{O}_{Y}$ is an $\mathcal{O}[G]$-bundle of rank one and hence gives rise to an element of $\operatorname{Pic}(\mathcal{O}[G])$. We say that an element of $\operatorname{Pic}(\mathcal{O}[G])$ is realisable if it can be obtained in this manner for some choice of unramified $G$-cover $f$. It is shown in $\left[\mathrm{A}, \mathrm{Bu}\right.$ ] that the subset $\mathfrak{R}_{X}(G)$ of realisable elements of $\operatorname{Pic}(\mathcal{O}[G])$ is in fact a subgroup and is moreover isomorphic 
to $H^{1}\left(X, \mathcal{W}_{n}\right)^{F=1}$. In this section, we shall apply the results of the present paper to study the subgroup $\mathfrak{R}_{X}(G)$ more closely.

Let $\mathcal{P}$ denote the Picard scheme $\operatorname{Pic}_{X / k}$ of $X / k$. Suppose that $\tau \in \mathfrak{R}_{X}(G)$, and that $\tau$ arises via an unramified $G$-cover $f: Y \rightarrow X$ as above. Corollary 7.2 (with $\left.d=p^{n}\right)$ gives an isomorphism $\operatorname{Pic}(\mathcal{O}[G]) \simeq \mathcal{P}\left(\boldsymbol{\mu}_{p^{n}}\right)$. Hence the class $\left(f_{*} \mathcal{O}_{Y}\right) \in$ $\operatorname{Pic}(\mathcal{O}[G])$ induces a morphism $\tau^{\prime}: \mu_{p^{n}} \rightarrow \mathcal{P}$. As $Y$ is a $G$-torsor over $X$, it follows from Section 1 of [Chi, M] that $\left(f_{*} \mathcal{O}_{Y}\right) \in \operatorname{Pic}(\mathcal{O}[G])$ is a primitive class, and so in fact $\tau^{\prime} \in \operatorname{Hom}\left(\boldsymbol{\mu}_{\boldsymbol{p}^{n}}, \mathcal{P}\right)$. The following result shows conversely that every primitive class is realisable (see also [A] for further results in this direction).

THEOREM 9.1. The composite bijection

$$
\operatorname{Pic}(\mathcal{O}[G]) \stackrel{\sim}{\rightarrow} \mathcal{P}\left(\boldsymbol{\mu}_{p^{n}}\right) \stackrel{\sim}{\rightarrow} \operatorname{Map}\left(\mu_{p^{n}}, \mathcal{P}\right)
$$

sends $\mathfrak{R}_{X}(G)$ onto $\operatorname{Hom}\left(\mu_{p^{n}}, \mathcal{P}\right)$.

Proof. Recall that $\mathfrak{R}_{X}(G) \simeq H^{1}\left(X, \mathcal{W}_{n}\right)^{F=1}$ (see Theorem 2.5 of $[\mathrm{A}, \mathrm{Bu}]$ ), and so the discussion above implies that $\#\left[H^{1}\left(X, \mathcal{W}_{n}\right)^{F=1}\right] \leqslant \# \operatorname{Hom}\left(\mu_{p^{n}}, \mathcal{P}\right)$. Following a suggestion of G. Pappas, we shall construct an injection $\operatorname{Hom}\left(\boldsymbol{\mu}_{\boldsymbol{p}^{n}}, \mathcal{P}\right) \rightarrow$ $H^{1}\left(X, \mathcal{W}_{n}\right)^{F=1}$. This will immediately imply the result we seek.

Let $\mathcal{P}_{F^{n}}$ denote the sub-group scheme of $F^{n}$-torsion on $\mathcal{P}$ over $k$, and write $\mathbf{Z} / p^{n} \mathbf{Z}$ for the Cartier dual of $\boldsymbol{\mu}_{\boldsymbol{p}^{n}}$. We begin by observing that since $\boldsymbol{\mu}_{\boldsymbol{p}^{n}}$ is annihilated by $F^{n}$, it follows that $\operatorname{Hom}\left(\boldsymbol{\mu}_{\boldsymbol{p}^{n}}, \mathcal{P}\right) \simeq \operatorname{Hom}\left(\boldsymbol{\mu}_{\boldsymbol{p}^{n}}, \mathcal{P}_{F^{n}}\right)$. Let $D\left(\mathcal{P}_{F^{n}}\right)$ denote the Cartier dual of the group scheme $\mathcal{P}_{F^{n}}$. Then, via Cartier duality, we have that $\operatorname{Hom}\left(\boldsymbol{\mu}_{\boldsymbol{p}^{\boldsymbol{n}}}, \mathcal{P}_{F^{n}}\right) \simeq \operatorname{Hom}\left(D\left(\mathcal{P}_{F^{n}}\right), \mathbf{Z} / p^{n} \mathbf{Z}\right)$.

Let $R=W(k)[F, V]$ be the Dieudonné ring over $W(k)$. (So, in $R$, we have $F V=V F=p, F a=\sigma(a) F$, and $a V=V \sigma(a)$ for any $a \in W(k)$, where $\sigma$ is the absolute Frobenius on $W(k)$.) Write $M\left(D\left(\mathcal{P}_{F^{n}}\right)\right)$ and $M\left(\mathbf{Z} / p^{n} \mathbf{Z}\right)$ for the Dieudonné modules of $D\left(\mathcal{P}_{F^{n}}\right)$ and $\mathbf{Z} / p^{n} \mathbf{Z}$, respectively (see $\S 3$ of [O] or Chap. III of [Fo]). Then $M\left(\mathbf{Z} / p^{n} \mathbf{Z}\right)$ is generated over $R$ by one element, and $(F-1) M\left(\mathbf{Z} / p^{n} \mathbf{Z}\right)$ $=0$.

Next, we note that it is shown in the proof of Theorem 4.4 of [O] that

$$
M\left(D\left(\mathcal{P}_{F^{n}}\right)\right) \simeq H^{1}\left(X, \mathcal{W}_{n}\right)
$$

Hence, it follows that there is an injection

$$
\operatorname{Hom}_{R}\left(M\left(\mathbf{Z} / p^{n} \mathbf{Z}\right), M\left(D\left(\mathcal{P}_{F^{n}}\right)\right)\right) \rightarrow H^{1}\left(X, \mathcal{W}_{n}\right)^{F=1} .
$$

Since the Dieudonné functor $M$ induces an anti-equivalence of categories between the category of commutative finite $k$-group schemes of $p$-power rank over $k$, and the category of left $R$-modules of finite $W(k)$-length, we have

$$
\operatorname{Hom}\left(D\left(\mathcal{P}_{F^{n}}\right), \mathbf{Z} / p^{n} \mathbf{Z}\right) \simeq \operatorname{Hom}_{R}\left(M\left(\mathbf{Z} / p^{n} \mathbf{Z}\right), M\left(D\left(\mathcal{P}_{F^{n}}\right)\right)\right),
$$

and so we finally deduce the existence of an injection $\operatorname{Hom}\left(\mu_{p^{n}}, \mathcal{P}\right) \rightarrow$ $H^{1}\left(X, \mathcal{W}_{n}\right)^{F=1}$, as required. 
This theorem completely describes $\mathfrak{R}_{X}(G)$ in terms of the Picard scheme of $X$. To end this section we explain how $\mathfrak{R}_{X}(G)$ can be computed more explicitly by using the Witt vector description of $\operatorname{Pic}(\mathcal{O}[G])$ given in Section 8. As an example, we shall then complete this computation in the case that $\# G=p$.

From now on, we assume only that $X$ is a separated scheme over $k$. We let $\pi^{1}(X, G)$ denote the group of isomorphism classes of $G$-torsors over $X$, and write $W_{n}(k)_{F-1}$ for the quotient $W_{n}(k) /(F-1) W_{n}(k)$. We fix a generator $g$ of $G$. The explicit computation of $\mathfrak{R}_{X}(G)$ in terms of Witt vectors is based upon the following result.

PROPOSITION 9.2. (a) There are short exact sequences (which are dependent upon the choice of $g$ )

$$
0 \rightarrow W_{n}(k)_{F-1} \rightarrow \pi^{1}(X, G) \rightarrow H^{1}\left(X_{e t}, \mathcal{W}_{n}\right)^{F=1} \rightarrow 0
$$

and

$$
0 \rightarrow W_{n}(k)_{F-1} \rightarrow \pi^{1}(X, G) \rightarrow \mathfrak{R}_{X}(G) \rightarrow 0 .
$$

(b) For each integer $i \geqslant 1$ the morphism $f: X_{e t} \rightarrow X_{\text {Zar }}$ which is induced by the identity map induces an isomorphism

$$
H^{1}\left(X, \mathcal{W}_{i}\right) \stackrel{\sim}{\rightarrow} H^{1}\left(X_{e t}, \mathcal{W}_{i}\right)
$$

Proof. The existence of (9.1) is established in ([S1], Prop. 13) and ([Mi], Chap. III, Prop. 4.12), while that of (9.2) is shown in ([A, Bu], Th. 2.5).

As a consequence of the Leray spectral sequence, (9.3) will follow if we prove that $R^{j} f_{*} \mathcal{W}_{i}=0$ for each $j>0$. This is proved for the case $i=1$ in ([Mi], Chap. III, Prop. 3.7) and in general can be proved by an induction on $i$ using the natural short exact sequence of sheaves

$$
0 \rightarrow \mathcal{W}_{i-1} \rightarrow \mathcal{W}_{i} \rightarrow \mathcal{W}_{1} \rightarrow 0
$$

We now view each $G$-torsor $Y$ over $X$ as being a sheaf on $X$ for the fpqc topology. Using the exactness of (9.1) we can give an explicit description of each such $Y$. To do this, we choose an affine open covering $\mathcal{U}=\left\{U_{i}: i \in I\right\}$ of $X$, with $U_{i}=\operatorname{Spec}\left(A_{i}\right)$, and we set $C^{0}\left(\mathcal{U}, \mathcal{W}_{n}\right):=\prod_{i \in I} \mathcal{W}_{n}\left(U_{i}\right)$. We let $Z^{1}\left(\mathcal{U}, \mathcal{W}_{n}\right)$ denote the set of Čech 1-cocycles of $\mathcal{W}_{n}$ with respect to $\mathcal{U}$.

It follows as a consequence of (9.1) and (9.3) that any $G$-torsor $Y$ over $X$ can be described by giving elements $\left(a_{i}\right) \in C^{0}\left(\mathcal{U}, \mathcal{W}_{n}\right)$ and $\left(z_{i, j}\right) \in Z^{1}\left(\mathcal{U}, \mathcal{W}_{n}\right)$ such that

$$
F z_{i, j}-z_{i, j}=a_{j}-a_{i}
$$

for all $i$ and $j$ in $I$ (cf. the discussion following [Mi], Chap. III, Prop. 4.12). More explicitly, for each index $i$ one has

$$
Y\left(U_{i}\right)=A_{i}\left[T_{1}, T_{2}, \ldots, T_{n}\right] / \mathbf{a}_{\mathbf{i}},
$$


where here $\left\{T_{r}: 1 \leqslant r \leqslant n\right\}$ is a set of independent indeterminates and $\mathbf{a}_{\mathbf{i}}$ denotes the ideal of the polynomial ring $A_{i}\left[T_{1}, T_{2}, \ldots, T_{n}\right]$ which is generated by those relations which are equivalent to the equality

$$
F\left(T_{1}, T_{2}, \ldots, T_{n}\right)-\left(T_{1}, T_{2}, \ldots, T_{n}\right)=a_{i},
$$

in $W_{n}\left(A_{i}\left[T_{1}, T_{2}, \ldots, T_{n}\right]\right)$. Since $X$ is separated, for each pair of indices $i$ and $j$ one has $U_{i} \cap U_{j}=\operatorname{Spec}\left(A_{i, j}\right)$, and the patching data for $Y$ is given by those isomorphisms

$$
A_{i, j}\left[T_{1}, T_{2}, \ldots, T_{n}\right] / \mathbf{a}_{\mathbf{i}} \rightarrow A_{i, j}\left[T_{1}, T_{2}, \ldots, T_{n}\right] / \mathbf{a}_{\mathbf{j}},
$$

which are induced by the translation

$$
\left(T_{1}, T_{2}, \ldots, T_{n}\right) \mapsto\left(T_{1}, T_{2}, \ldots, T_{n}\right)+z_{i, j},
$$

in $W_{n}\left(A_{i, j}\left[T_{1}, T_{2}, \ldots, T_{n}\right]\right)$. The action of $G$ on each $Y\left(U_{i}\right)$ is specified so that $g$ corresponds to the $A_{i}$-algebra automorphism which acts on each $T_{r}$ so as to induce the translation

$$
\left(T_{1}, T_{2}, \ldots, T_{n}\right) \mapsto\left(T_{1}, T_{2}, \ldots, T_{n}\right)+1
$$

in $W_{n}\left(A_{i}\left[T_{1}, T_{2}, \ldots, T_{n}\right]\right)$. Via this action, we obtain a $G$-torsor $f: Y \rightarrow X$ whose image in $H^{1}\left(X, \mathcal{W}_{n}\right)^{F=1}$ under the map given by (9.1) and (9.3) is represented by the 1-cocycle $\left(z_{i, j}\right)$.

We now aim to describe the image of the element of $\operatorname{Pic}(\mathcal{O}[G])$ which corresponds to $f_{*} \mathcal{O}_{Y}$ under the isomorphism $\omega_{g}$ of (8.3). To do this, we use the following result.

LEMMA 9.3. Let $G$ act on $Y\left(U_{i}\right)$ as above. Then $Y\left(U_{i}\right)$ is a free $A_{i}[G]$-module, with basis element $\left(\prod_{r=1}^{n} T_{r}\right)^{p-1}$.

Proof. Write $\alpha_{m}:=\left(\prod_{r=1}^{m} T_{r}\right)^{p-1}$ (where $1 \leqslant m \leqslant n$ ), and for each subgroup $H$ of $G$, set $\operatorname{Tr}_{H}\left(\alpha_{m}\right):=\sum_{h \in H} h\left(\alpha_{m}\right)$. To prove the desired result, it suffices to show that $\operatorname{Tr}_{G}\left(\alpha_{n}\right) \in A_{i}^{*}$ (cf. the proof of Lemma 2.3 of [A, Bu]).

Now for each $j \in \mathbb{Z},\left(g^{p^{n-1}}\right)^{j}$ sends $\alpha_{n}$ to $\left[\left(T_{n}+j\right) \prod_{r=1}^{n-1} T_{r}\right]^{p-1}$. Hence,

$$
\begin{aligned}
& \operatorname{Tr}_{<g p^{n-1}>}\left(\alpha_{n}\right)=\sum_{j=0}^{p-1}\left[\left(T_{n}+j\right) \prod_{r=1}^{n-1} T_{r}\right]^{p-1} \\
& =\alpha_{n-1} \sum_{j=0}^{p-1}\left(T_{n}+j\right)^{p-1} \text {. }
\end{aligned}
$$

We have

$$
\sum_{j=0}^{p-1}\left(T_{n}+j\right)^{p-1}=\sum_{j=0}^{p-1} \sum_{s=0}^{p-1}\left(\begin{array}{c}
p-1 \\
s
\end{array}\right) T_{n}^{p-1-s} j^{s}
$$




$$
\begin{aligned}
& =\sum_{s=0}^{p-1}\left(\begin{array}{c}
p-1 \\
s
\end{array}\right) T_{n}^{p-1-s} \sum_{j=0}^{p-1} j^{s} \\
& =-1,
\end{aligned}
$$

where the last equality follows from the fact that

$$
\sum_{j=0}^{p-1} j^{s} \equiv\left\{\begin{array}{lrl}
0 \quad(\bmod p), & \text { if } s \neq \equiv p-1 \quad(\bmod p) \\
-1 \quad(\bmod p), & \text { otherwise }
\end{array}\right.
$$

Hence we have that $\operatorname{Tr}_{<g^{p^{n-1}}>}\left(\alpha_{n}\right)=-\alpha_{n-1}$. It therefore follows by induction on $n$ that $\operatorname{Tr}_{G}\left(\alpha_{n}\right)=(-1)^{n} \in A_{i}^{*}$, and this establishes the required result.

For each integer $r \in\{1,2, \ldots, n\}$ we define an element $T_{r}\left(z_{i, j}\right)$ of $A_{i, j}$ $\left[T_{1}, T_{2}, \ldots, T_{n}\right]$ by means of the equality

$$
\left(T_{1}, T_{2}, \ldots, T_{n}\right)+z_{i, j}=\left(T_{1}\left(z_{i, j}\right), T_{2}\left(z_{i, j}\right), \ldots, T_{n}\left(z_{i, j}\right)\right)
$$

in $\mathrm{W}_{n}\left(A_{i, j}\left[T_{1}, T_{2}, \ldots, T_{n}\right]\right)$. An argument similar to that of Lemma 9.3 shows that the element $\left(\prod_{r=1}^{n} T_{r}\left(z_{i, j}\right)\right)^{p-1}$ is a free generator of $Y\left(U_{i} \cap U_{j}\right)$ over $A_{i, j}[G]$. Hence there exists an element $\lambda_{i, j} \in A_{i, j}[G]^{*}$ such that

$$
\left(\prod_{r=1}^{n} T_{r}\left(z_{i, j}\right)\right)^{p-1}=\lambda_{i, j}\left(\prod_{r=1}^{n} T_{r}\right)^{p-1} \in Y\left(U_{i} \cap U_{j}\right) .
$$

Since $Y\left(U_{i}\right)=f_{*} \mathcal{O}_{Y}\left(U_{i}\right)$, the 1-cocycle $(i, j) \mapsto \lambda_{i, j}$ is a representative of the isomorphism class of $f_{*} \mathcal{O}_{Y}$ in $H^{1}\left(X, \mathcal{O}[G]^{*}\right)$. After decomposing each element $\lambda_{i, j}$ using the Artin-Hasse exponential as in (8.1) we therefore obtain a representative for the image of $\left(f_{*} \mathcal{O}_{Y}\right)$ in $\mathrm{W}(\mathcal{O}[G])$ under the isomorphism $\omega_{g}$ of (8.3).

As an explicit example, we shall now complete this calculation in the case that $\# G=p$. We observe that in this case the equality (9.4) takes the form

$$
\left(T+z_{i, j}\right)^{p-1}=\lambda_{i, j} T^{p-1} \in A_{i, j}[T] /\left(T^{p}-T-a_{i}\right)
$$

(where here we write $T$ in place of $T_{1}$ ).

LEMMA 9.4. For each pair of indices $i, j$ and for each element $z$ of $A_{i, j}$ one has

$$
\begin{aligned}
(T+z)^{p-1}= & \left(1+\sum_{r=1}^{p-1}\left(\frac{\prod_{s=0}^{r-1}(z-s)}{r !}\right)(g-1)^{r}\right) T^{p-1} \\
& \in A_{i, j}[T] /\left(T^{p}-T-a_{i}\right) .
\end{aligned}
$$

Proof. For any subset $\left\{c_{r}: 0 \leqslant r<p\right\}$ of $A_{i, j}$ one has

$$
\left(\sum_{r=0}^{p-1} c_{r} g^{r}\right) T^{p-1}=\sum_{r=0}^{p-1} c_{r}(T+r)^{p-1} \in A_{i, j}[T] /\left(T^{p}-T-a_{i}\right) .
$$


Hence we must solve the equation

$$
(T+z)^{p-1}=\sum_{r=0}^{p-1} c_{r}(T+r)^{p-1} \in A_{i, j}[T] /\left(T^{p}-T-a_{i}\right)
$$

Since the maximum exponent of $T$ which occurs on both sides of this equality is less than $p$, we can equate coefficients of powers of $T$. This yields the system of equations

$$
z^{k}=\sum_{r=0}^{p-1} c_{r} r^{k}, \quad(0 \leqslant k<p)
$$

(where we interpret $0^{0}$ as 1 ). For each integer $r$ with $0 \leqslant r<p$ we define $c_{r}^{\prime}=$ $\sum_{s=r}^{p-1} c_{s}\left(\begin{array}{l}s \\ r\end{array}\right)$. Then we have

$$
\sum_{r=0}^{p-1} c_{r} g^{r}=\sum_{r=0}^{p-1} c_{r}(1+(g-1))^{r}=\sum_{r=0}^{p-1} c_{r}^{\prime}(g-1)^{r}
$$

To complete the proof of the result, we must therefore show that $c_{0}^{\prime}=1$ and that $c_{r}^{\prime}=(1 / r !) \prod_{s=0}^{r-1}(z-s)$ for each integer $r$ with $0<r<p$. The equality $c_{0}^{\prime}=1$ follows immediately from (9.5) with $k=0$, but to deal with the case $r>0$ we shall need some further notation.

For integers $a, b$ with $0 \leqslant a \leqslant b$, we write $\sigma_{(a)}(b)$ for the value of the $a$-th symmetric polynomial in $b$ variables evaluated on the set of integers $\{1,2, \ldots, b\}$. Let $Z$ be an indeterminate. Then the following identity holds in $\mathbb{Z}[Z]$ :

$$
\prod_{s=0}^{r-1}(Z-s)=\sum_{s=0}^{r-1} Z^{r-s}(-1)^{s} \sigma_{(s)}(r-1), \quad(r \geqslant 1) .
$$

For each integer $r$ with $0<r<p$ we then have

$$
\begin{aligned}
c_{r}^{\prime} & =\sum_{s=r}^{p-1} c_{s}\left(\begin{array}{l}
s \\
r
\end{array}\right) \\
& =\sum_{s=r}^{p-1} \frac{c_{s}}{r !} s(s-1) \ldots(s-(r-1)) \\
& =\sum_{s=1}^{p-1} \frac{c_{s}}{r !} s(s-1) \ldots(s-(r-1)) \\
& =\sum_{s=1}^{p-1} \frac{c_{s}}{r !} \sum_{j=0}^{r-1} s^{r-j}(-1)^{j} \sigma_{(j)}(r-1) \quad(\operatorname{using}(9.6))
\end{aligned}
$$




$$
\begin{aligned}
& =\sum_{j=0}^{r-1} \frac{(-1)^{j}}{r !} \sigma_{(j)}(r-1) \sum_{s=1}^{p-1} c_{s} s^{r-j} \\
& =\sum_{j=0}^{r-1} \frac{(-1)^{j}}{r !} \sigma_{(j)}(r-1) z^{r-j} \quad(\operatorname{using}(9.5)) \\
& =\frac{1}{r !} \prod_{j=0}^{r-1}(z-j) \quad(\operatorname{using}(9.6)) .
\end{aligned}
$$

This completes the proof of the lemma.

The next result gives the Artin-Hasse decomposition (see (8.1) and (8.2)) of the element of $A_{i, j}[G]$ which occurs on the right-hand side of the equality in the statement of Lemma 9.4. We write $\exp _{(p-1)}(-)$ for the truncated exponential map $x \mapsto \sum_{s=0}^{p-1} x^{s} / s !$

LEMMA 9.5. For each pair of indices $i, j$ and for each element $z$ of $A_{i, j}$ one has

$$
\begin{aligned}
1 & +\sum_{r=1}^{p-1}\left(\frac{\prod_{s=0}^{r-1}(z-s)}{r !}\right)(g-1)^{r} \\
& =\prod_{r=1}^{p-1} \exp _{(p-1)}\left(z \cdot\left(\frac{(-1)^{r-1}}{r}\right)(g-1)^{r}\right) \in A_{i, j}[G] .
\end{aligned}
$$

Proof. Let $Z_{1}$ and $Z_{2}$ be any two independent indeterminates. Then we have the following identity of formal power series in $\mathbb{Q}\left[\left[Z_{1}, Z_{2}\right]\right]$ :

$$
\exp \left(Z_{1} \log \left(1+Z_{2}\right)\right)=1+\sum_{r \geqslant 1}\left(\frac{Z_{2}^{r}}{r !}\right) \prod_{s=0}^{r-1}\left(Z_{1}-s\right) .
$$

(A proof of this will be given below.) By applying this identity with $Z_{1}=z$ and $Z_{2}=g-1$, and using the fact that $Z_{2}^{p}=0 \in A_{i, j}[G]$, we obtain the equality

$$
\begin{aligned}
1 & +\sum_{r=1}^{p-1}\left(\frac{\prod_{s=0}^{r-1}(z-s)}{r !}\right)(g-1)^{r} \\
& =\exp _{(p-1)}\left(z \cdot \sum_{r=1}^{p-1}\left(\frac{(-1)^{r-1}}{r}\right)(g-1)^{r}\right) \in A_{i, j}[G] .
\end{aligned}
$$

This is equivalent to the desired equality (9.7).

To complete the argument, we now give a proof of the identity (9.8). 
We set

$$
\begin{aligned}
& \alpha\left(Z_{1}, Z_{2}\right):=\exp \left(Z_{1} \log \left(1+Z_{2}\right)\right) \\
& \beta\left(Z_{1}, Z_{2}\right):=1+\sum_{r \geqslant 1}\left(\frac{Z_{2}^{r}}{r !}\right) \prod_{s=0}^{r-1}\left(Z_{1}-s\right) .
\end{aligned}
$$

A routine computation yields identities of formal power series

$$
\begin{aligned}
& \frac{\partial \alpha}{\partial Z_{2}}=\frac{Z_{1}}{1+Z_{2}} \alpha, \\
& \left(1+Z_{2}\right) \frac{\partial \beta}{\partial Z_{2}}=Z_{1} \beta .
\end{aligned}
$$

These imply that $\partial / \partial Z_{2}[\beta / \alpha]$ is identically 0 , from which we deduce that $\beta / \alpha$ is independent of $Z_{2}$. The identity (9.8) now follows upon noting that the series $\alpha\left(Z_{1}, 0\right)$ and $\beta\left(Z_{1}, 0\right)$ are both identically 1 .

The following description of $\mathfrak{R}_{X}(G)$ is now a consequence of Lemmas 9.4 and 9.5 , together with the discussion preceding them.

PROPOSITION 9.6. Let $X$ be any separated scheme over $k$. If $\# G=p$, then

$$
\begin{aligned}
\omega_{g}\left(\Re_{X}(G)\right) & =\left\{\left(0, c,-2^{-1} c, 3^{-1} c, \ldots,-(p-1)^{-1} c\right): c \in H^{1}(X, \mathcal{O})^{F=1}\right\} \\
& \subseteq \mathrm{W}(\mathcal{O}[G])
\end{aligned}
$$

for any generator $g$ of $G$.

Proposition 9.6 suggests the following question. Let $G$ be a cyclic group of order $p^{n}$ with $n \geqslant 1$. If $g$ is a generator of $G$, then just as in (8.2), the Artin-Hasse exponential induces an isomorphism

$$
L_{g}: k[G]^{*} \stackrel{\sim}{\rightarrow} k^{*} \times \prod_{\substack{0<i<p^{n} \\(i, p)=1}} W_{r_{i}(n)}(k) .
$$

We define Witt vectors $a_{i} \in W_{r_{i}(n)}(k)$ by the equality

$$
L_{g}(g)=1 \times \prod_{\substack{0<i<p^{n} \\(i, p)=1}} a_{i}
$$

Note that since each $\mathcal{W}_{r_{i}(n)}$ is a sheaf of $W_{r_{i}(n)}(k)$-algebras, it follows that $H^{1}\left(X, \mathcal{W}_{r_{i}(n)}\right)$ is a $W_{r_{i}(n)}(k)$-module. We may therefore define a morphism

$$
\phi_{g}: H^{1}\left(X, \mathcal{W}_{n}\right)^{F=1} \rightarrow \mathrm{W}(\mathcal{O}[G])
$$


by setting

$$
\phi_{g}(c)=\left(0, \prod_{\substack{0<i<p^{n} \\(i, p)=1}}\left(a_{i} \cdot R_{n, r_{i}(n)}(c)\right)\right) .
$$

(Recall (see the beginning of Section 9) that $R_{n, r_{i}(n)}$ denotes the map induced on cohomology by the restriction map on Witt vectors.)

QUESTION 9.7. Let $g$ be any generator of G. Is $\omega_{g}\left(\Re_{X}(G)\right)=\operatorname{Im}\left(\phi_{g}\right)$ ?

Proposition 9.6 implies that Question 9.7 has an affirmative answer if $n=1$. It would be interesting to know whether this is still the case if $n>1$.

\section{Acknowledgements}

We are very grateful to T. Chinburg, G. Pappas, D. Solomon and particularly to D. Grayson for illuminating discussions and correspondence concerning this work. We owe a great deal to $\mathrm{B}$. Köck for carefully reading an earlier version of this paper and for giving us a large number of very helpful comments. A. Agboola was partially supported by the NSF. He is also very grateful to the Institute for Advanced Study, Princeton, where a portion of this work was carried out.

\section{References}

[A] Agboola, A.: On primitive and realisable classes, Compositio Math. 126 (2001), 113122.

[A,Bu] Agboola, A. and Burns, D.: On the Galois structure of equivariant line bundles on curves, Amer. J. Math. 120 (1998). 1121-1163.

[At] Atiyah, M. F.: On the Krull-Schmidt theorem with application to sheaves, Bull. Soc. Math. France 84 (1956) 307-317 (or Collected Papers Vol. I, 5).

[B] Bass, H.: Algebraic K-theory, W. A. Benjamin, New York, 1968.

[Bo] Bourbaki, N.: Commutative Algebra, Hermann, Paris, 1972.

[Bu,Ch] Burns, D. and Chinburg, T.: On Adams operations and integral hermitian Galois representations, Amer. J. Math. 118 (1996), 925-962.

[Cha] Chapman, R.: Classgroups of sheaves of locally-free modules over global function fields, In: D. Goss (ed.), The Arithmetic of Function Fields, Ohio State Univ. Math. Res. Inst. Publ. 2, de Gruyter, Berlin, 1992.

[Chi,M] Childs, L. and Magid, A.: The Picard invariant of a principal homogeneous space, $J$. Pure Appl. Algebra 4 (1974), 273-286.

[Ch] Chinburg, T.: Galois structure of de Rham cohomology of tame covers of schemes, Ann. Math. 139 (1994), 443-490.

[Ch,E] Chinburg, T. and Erez, B.: Equivariant Euler-Poincaré characteristics and tameness, In: Proc. Journées Arithmétiques 1991, Astérisque, Soc. Math. France, Paris, 1992.

[Ch,E,P,T1] Chinburg, T., Erez, B., Pappas, G. and Taylor, M. J.: Epsilon constants and the Galois structure of de Rham cohomology, Ann. of Math. 146 (1997), 411-473. 
[Ch,E,P,T2] Chinburg, T., Erez, B., Pappas G. and Taylor, M. J.: Riemann-Roch type theorems for schemes with a finite group action, Crelle 489 (1997), 151-187.

[C,R1] Curtis, C. and Reiner, I.: Methods of Representation Theory, Vol. I, Wiley, New York, 1981.

[C,R2] Curtis, C. and Reiner, I.: Methods of Representation Theory, Vol. II, WileyInterscience, New York, 1987.

[D,Gr] Dieudonné, J. and Grothendieck, A.: Eléments de géométrie algébrique I, Springer, Berlin, 1971.

[Fo] Fontaine, J.-M.: Groupes p-divisibles sur les corps locaux, Asterisque 47-48 (1977).

[Fr] Fröhlich, A.: Galois Module Structure of Algebraic Integers, Ergeb. Math. Wiss. 1, Springer, Berlin, 1983.

[Fr,T] Fröhlich A. and Taylor, M. J.: Algebraic Number Theory, Cambridge Univ. Press, 1991.

[Fu,L] Fulton, W. and Lang, S.: Riemann-Roch Algebra, Springer, New York, 1985.

[G] Gersten, S.: The localization theorem for projective modules, Comm. Algebra 2 (1974), 307-350

[Gi] Giraud, J.: Cohomologie non abelienne, Springer, Heidelberg, 1971.

[Gra] Grayson, D.: Higher algebraic K-theory II (after Quillen), In: Algebraic K-theory (Northwestern University 1976), Lecture Notes in Math. 551, Springer, Berlin, 1976.

[H] Hartshorne, R.: Algebraic Geometry, Grad. Text in Math. 52, Springer, New York, 1977.

[Mi] Milne, J.: Étale Cohomology, Princeton Math. Ser. 33, Princeton University Press, 1980.

[M] Mumford, D.: Lectures on Curves on an Algebraic Surface, Princeton University Press, 1966.

[M,F] Mumford, D. and Fogarty, J.: Geometric Invariant Theory, Springer, New York, 1982.

[O] Oda, T.: The first de Rham cohomology group and Dieudonné modules, Ann. Sci. Ecole Norm. Sup. 2 (1969), 63-135.

[Q] Quillen, D.: Higher algebraic K-theory I, In: Algebraic K-theory (Batelle Institute Conference 1972), Lecture Notes in Math. 341, Springer, Berlin, 1973.

[R] Reiner, I. Maximal Orders, Academic Press, London, 1975.

[S1] Serre, J.-P.: Sur la topologie des variétés algèbraiques en caracteristique $p$, In: Sympos. Internacional de Topologie Algebraica, Universidad Nacional Autónoma de México and UNESCO, Mexico City, 1958, pp. 24-53.

[S2] Serre, J.-P.: Algebraic Groups and Class Fields, Springer, Berlin, 1988.

[Si] Silvester, J. R.: Introduction to Algebraic K-theory, Chapman and Hall, London, 1981.

[Sr] Srinivas, V.: Algebraic K-theory, 2nd edn, Birkhäuser, Boston, 1996. 
